\title{
FIELD DEPLOYABLE DYNAMIC LIGHTING SYSTEM FOR TURBID WATER IMAGING
}

\author{
By \\ Geoffrey Allen Gorman \\ B.S., University of North Florida, 2004 \\ Submitted in partial fulfillment of the requirements for the degree of \\ Master of Science \\ at the \\ MASSACHUSETTS INSTITUTE OF TECHNOLOGY \\ and the \\ WOODS HOLE OCEANOGRAPHIC INSTITUTION
}

September 2011

Signature of Author
Joint Program in Oceanography / Applied Ocean Science and Engineering Massachusetts Institute of Technology and Woods Hole Oceanographic Institution August 17, 2011

Certified by

Hanumant Singh Thesis Supervisor

Accepted by

James C. Preisig Chair, Joint Committee for Applied Ocean Science and Engineering Massachusetts Institute of Technology/ Woods Hole Oceanographic Institution

Accepted by

David E. Hardt

Chair, Committee on Graduate Students - Mechanical Engineering Massachusetts Institute of Technology 


\section{(C) 2011 Geoffrey Gorman \\ All rights reserved.}

The author hereby grants to MIT and WHOI permission to reproduce and to distribute publicly paper and electronic copies of this thesis document in whole or in part in any medium now known or hereafter created. 
Field Deployable Dynamic Lighting System for Turbid Water Imaging

by

Geoffrey Allen Gorman

Submitted to the Department of Mechanical Engineering and the Applied Ocean Physics and Engineering Department in partial fulfillment of the requirements for the degree of Master of Science in Oceanographic Engineering

\begin{abstract}
The ocean depths provide an ever changing and complex imaging environment. As scientists and researches strive to document and study more remote and optically challenging areas, specifically scatter-limited environments. There is a requirement for new illumination systems that improve both image quality and increase imaging distance.

One of the most constraining optical properties to underwater image quality are scattering caused by ocean chemistry and entrained organic material. By reducing the size of the scatter interaction volume, one can immediately improve both the focus (forward scatter limited) and contrast (backscatter limited) of underwater images. This thesis describes a relatively simple, cost-effective and field-deployable low-power dynamic lighting system that minimizes the scatter interaction volume with both subjective and quantifiable improvements in imaging performance.
\end{abstract}

Thesis Supervisor: Hanumant Singh

Title: Associate Scientist with Tenure

Woods Hole Oceanographic Institution 


\section{Dedication}

This paper is dedicated to my family. Their support, patience and inspiration were a crucial part of my ability to accomplish this thesis and complete my late blooming academic endeavors. 
Acknowledgements

I want to especially thank my thesis advisor, Dr. Hanumant Singh, for his continued efforts to keep me on track and ensure that I received the most fulfilling education and research program that I could. Hanu is truly a pleasure to work with. His easy-going attitude coupled with a serious commitment to the endeavors of the Joint Program are commendable and made the time I spent at both WHOI and MIT fly by. I look forward to working with him in the future.

I would also like to thank my original office mate, Derya Akkaynak Yellin, for keeping me in line, not being afraid to telling me how it really is and being a good friend.

I appreciate the friendship and assistance from each member of the Singh Research Group; Clay Kunz, Chris Murphy, Jeff Kaeli and Scott Haven. Each of them, in their own way, made this thesis possible.

Thank you to the Academic Programs Office for fielding numerous questions, comments and complaints. Your professionalism, dedication and support are greatly appreciated.

And finally, I thank the United States Navy, the Office of the Oceanographer of the Navy, and the Naval Post-Graduate School for the opportunity to participate in this excellent program. 


\section{CONTENTS}

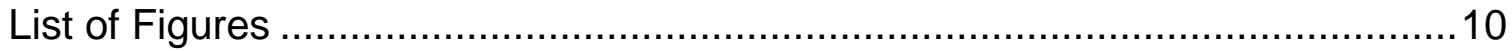

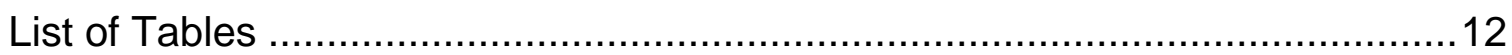

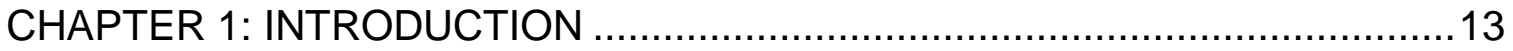

1.1 Why is underwater imaging is difficult? ...........................................13

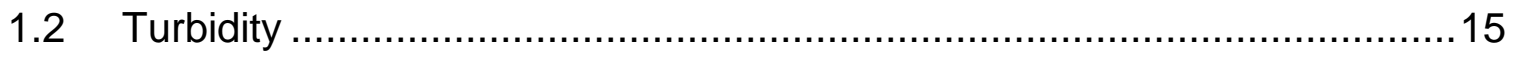

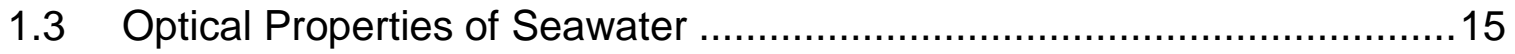

1.3.1 Inherent Optical Properties .......................................................18

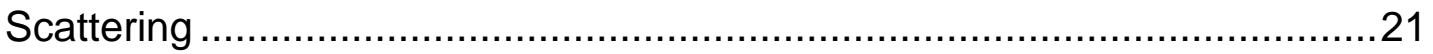

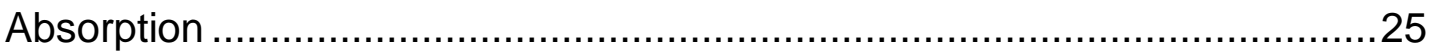

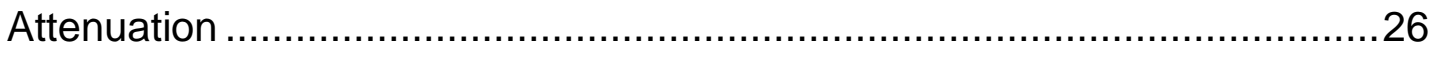

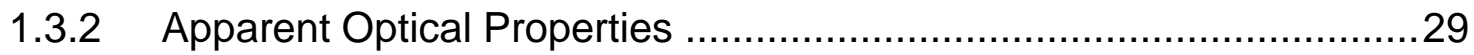

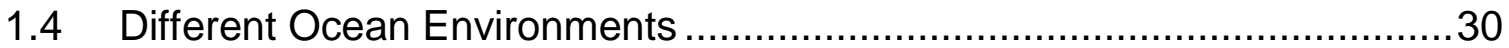

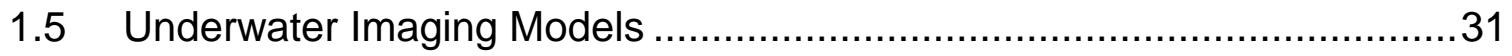

CHAPTER 2: IMAGING IN TURBID ENVIRONMENTS..................................33

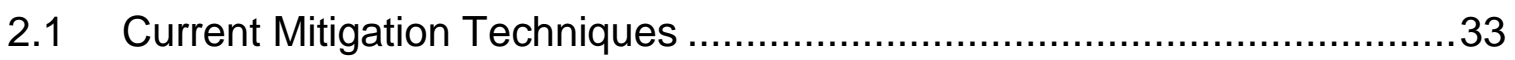

2.1.1 Source and receiver separation (SRS) …......................................3

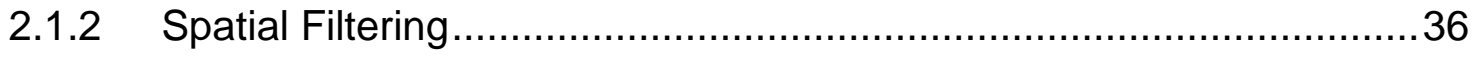

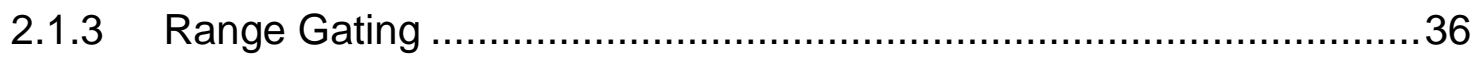

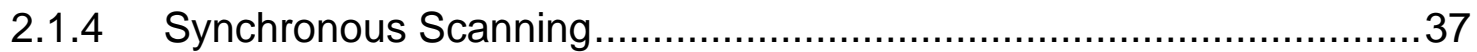

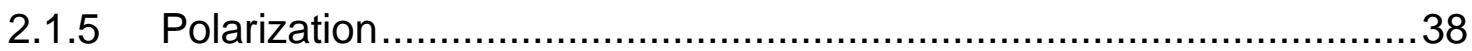

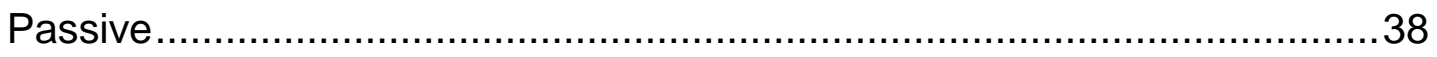

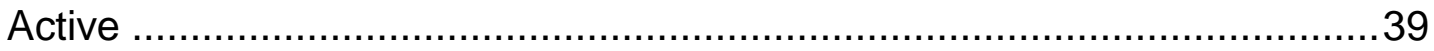

2.1.6 Modulated waveform illumination ................................................ 40

2.1.7 High Frequency Illumination .......................................................40

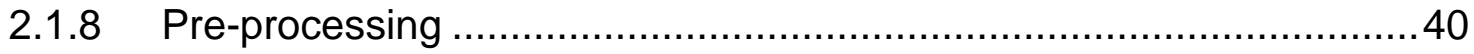


2.1.9 Confocal imaging [Levoy \& Singh] ........................................... 41

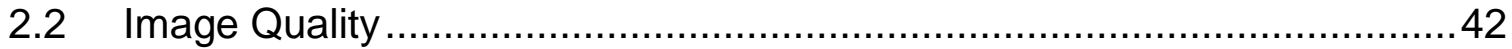

CHAPTER 3: THE DYNAMIC LIGHTING SYSTEM................................... 45

3.1 Environmental Design Constraints .......................................................45

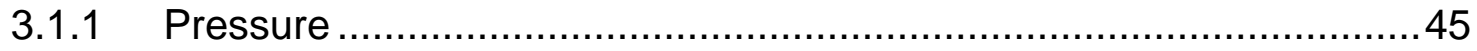

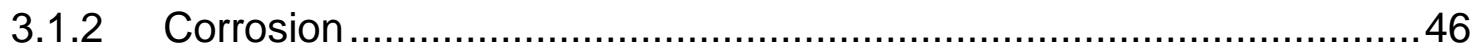

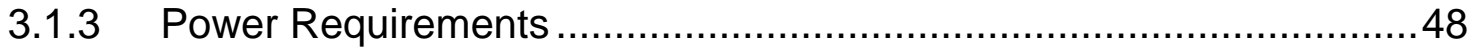

3.2 Scatter and Illumination Volumes .........................................................50

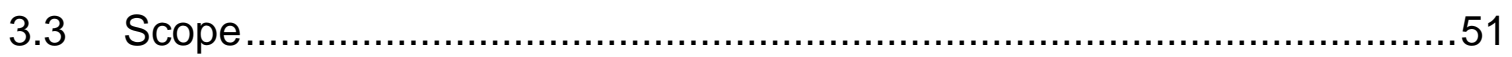

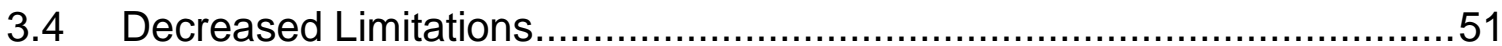

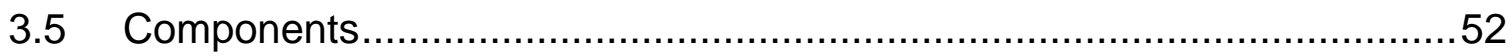

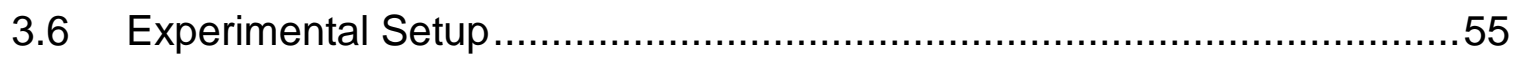

CHAPTER 4: DATA COLLECTION AND ANALYSIS …................................57

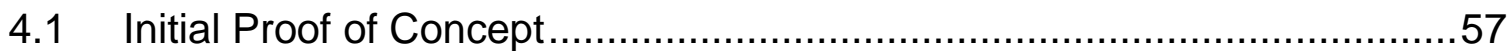

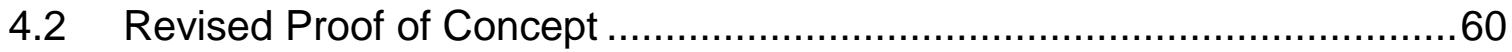

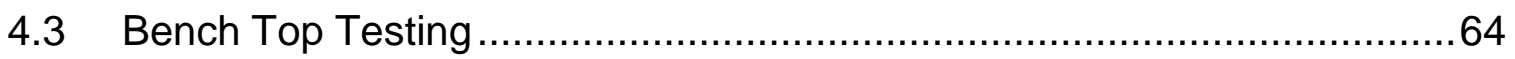

4.3.1 Images and Quantitative Results ...............................................68

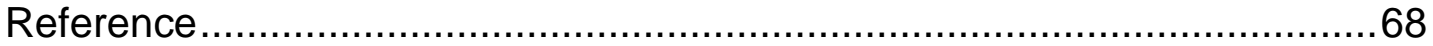

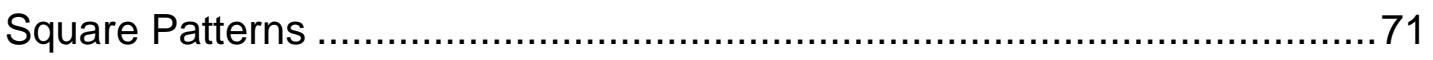

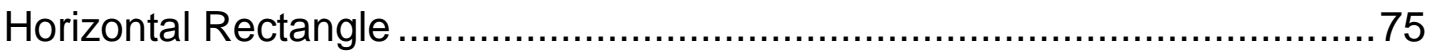

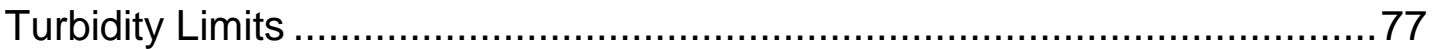

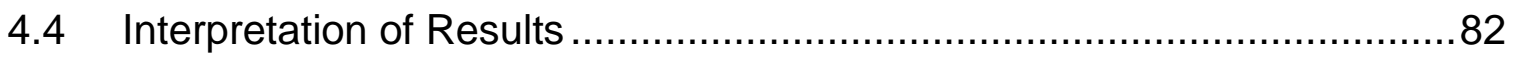


CHAPTER 5: CONCLUSIONS AND FUTURE WORK ….............................. 85

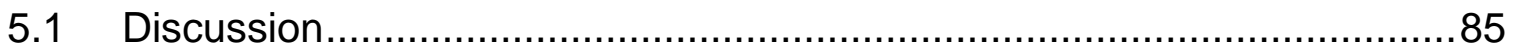

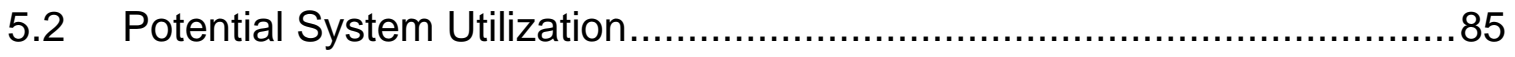

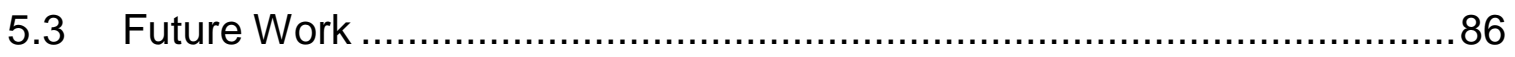

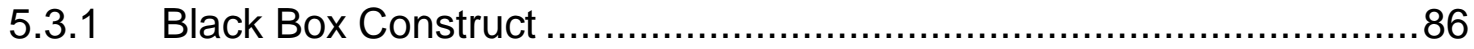

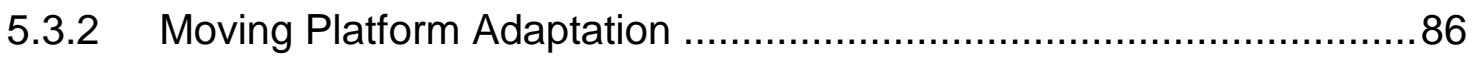

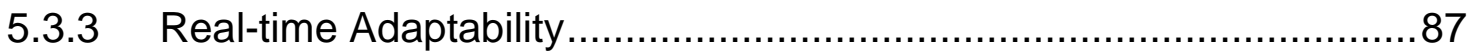

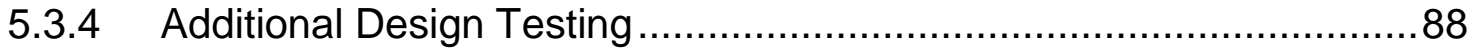

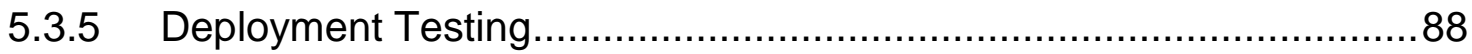

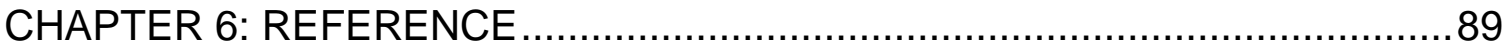

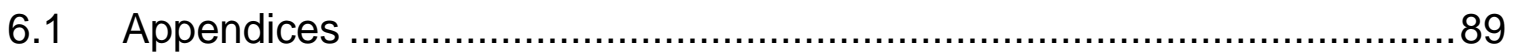

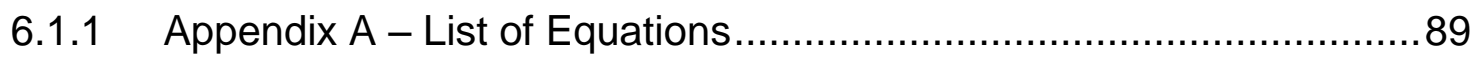

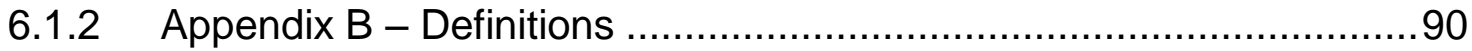

6.1.3 Appendix C - System Component Specifications.............................94

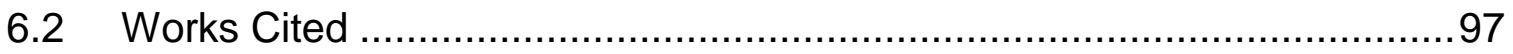




\section{List of Figures}

Figure 1- Composite global ocean (and land) color image $\ldots \ldots \ldots \ldots \ldots \ldots \ldots \ldots \ldots \ldots \ldots . . . .17$

Figure 2 - Inherent optical property relationships .........................................20

Figure 3 - Volume scattering function for two disparate water bodies ...............22

Figure 4 - Backscatter contribution to image quality.....................................24

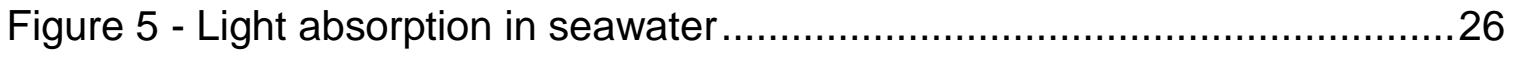

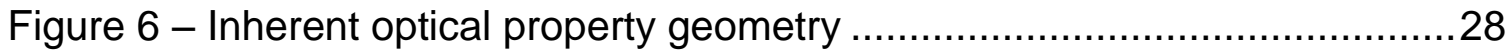

Figure 7 - Scatter interaction volume differences...........................................34

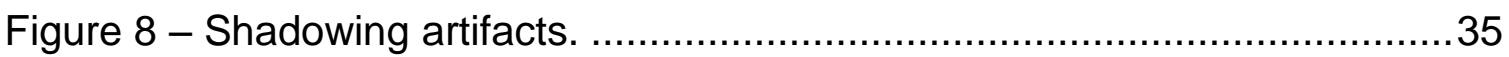

Figure 9 - Backscatter and target-reflected signals .....................................37

Figure 10 - Corrosion potential for various materials..................................47

Figure 12 - Dynamic scatter interaction volume based on dynamic lighting.......50

Figure 13 - Beagle board / pico projector original developer block diagram.......53

Figure 14 - Beagle board and pico projector shown in actual size ...................53

Figure 15 - Initial dynamic lighting system prototype...................................54

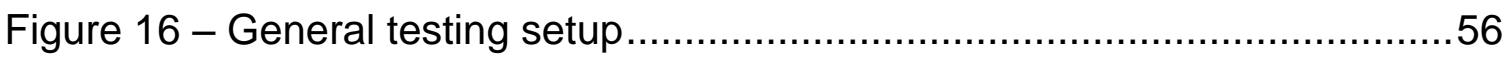

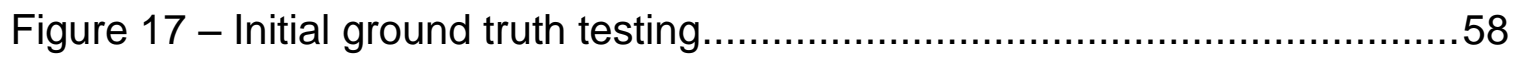

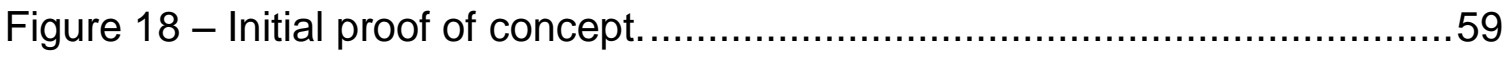

Figure 19 - Initial gradient magnitude comparison .........................................60

Figure 20 - Revised ground truth testing ...................................................

Figure 21 - Revised proof of concept ……..............................................62

Figure 22 - Revised gradient magnitude comparison ....................................63 
Figure 23 - Bench top testing experimental setup ......................................64

Figure 24 - Image and quantitative results layout......................................67

Figure 25 - Center gradient magnitude comparison image .............................67

Figure 26 - Horizontal rectangle pattern ................................................. 75

Figure 27 - Dynamic lighting sweeping speed effect on contrast .....................83

Figure 28 - Dynamic lighting area effect on contrast.....................................83

Figure 29 - Dynamic lighting system 2.0 (DLS 2.0) ..................................... 87 


\section{List of Tables}

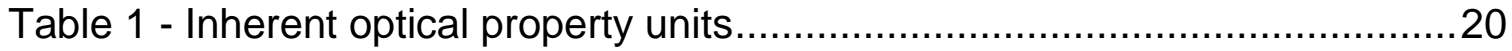

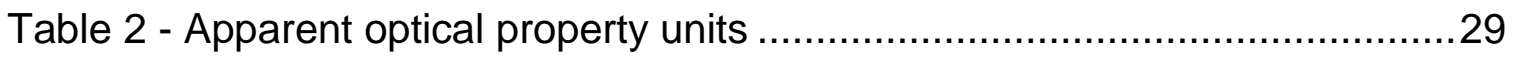

Table 3 - Lighting characteristics for various imaging platforms.......................36

Table 4 - Subjective image quality assessment techniques ............................43

Table 5 - Subjective image quality assessment techniques ............................44

Table 6 - Summary of bench top testing results.........................................82 
Keywords: Imaging, turbidity, turbid water, scatter, backscatter, forward scatter, absorption, attenuation, dynamic lighting, underwater vehicle

\section{CHAPTER 1: INTRODUCTION}

\subsection{Why underwater imaging is difficult}

In order to design and construct an illumination system used at ocean depths, one must understand and attempt to overcome the limitations of the underwater optical environment. For many years, scientists and researchers have attempted to unlock the secrets of the world's oceans; a feat that can be obtained by optical imaging. As technology advances and our eyes look towards deeper and towards more challenging environments, one thing remains absolute: doing anything underwater is more difficult, especially imaging.

Clear, concise and geographically accurate images, are an integral part of ocean documentation and are a major source of oceanographic knowledge. Although, many years of research have been devoted to solving the challenges to underwater imaging, as technology develops and one concept is mastered, two more take its place. In an environment as complex as the ocean, assumptions must be made and as research progresses, they must evolve as well. For example, the well-known and documented assumptions to ocean modeling, concepts dating from 1962 [Duntley], have been challenged. Jaffe states that the assumptions of linearity and small-angle-scattering theory are only valid at

relatively short attenuation distances [Jaffe 1995]. This brief, but concise proof 
by Jaffe laid 30 years of assumptions on the table and limited their scope by providing more accurate boundaries. It is this slowly evolving knowledge that is both a hindrance to understanding and a door of opportunity to increase our understanding.

This thesis provides a means to new insights in ocean optics and imaging. By increasing our understanding of the oceanic environment in a novel and physical way, I hope to provide a large door of opportunity while decreasing the avenue for challenges to previously held understanding. The background and nascent understanding of the ocean environment, for the purposes of this thesis, will consist of four generalized topics; turbidity, underwater optical properties (both inherent and apparent), ocean environments and imaging models. These topics are provided specifically to accentuate current understanding and ultimately provide better underwater imaging. 


\subsection{Turbidity}

The turbidity or cloudiness of seawater is an important consideration for any design of an underwater imaging system. The levels of seawater cloudiness are measured in turbidity units, either Nephelometric (NTU) or Jackson (JTU). Both unit types are used interchangeably and are roughly equivalent in magnitude. The average person perceives turbidity in water at greater than 5 NTU [Myre \& Shaw].

In the ocean turbidity is caused by dissolved and particulate matter. This presence and concentration of such matter most heavily influences the optical components of the ocean and for the most part determines the optical properties of the environment [Gutierrez, et al]. The organisms and particles in a volume of seawater are continually changing, thereby changing the turbidity and optical properties of the imaging environment.

Currently, most optical research on turbidity and its effect is concentrated on atmospheric concerns, as opposed to those related to the ocean [Narasimhan, et al]. In this thesis I concentrate on the steps necessary to successfully design an illumination system that will operate in a turbid environment as experienced in the world's oceans.

\subsection{Optical Properties of Seawater}

The firm understanding of the optical properties of seawater is the most important facet to the design and implementation of underwater imaging schemes. 
Decades of research resulting in numerous publications are available to the researcher [Duntley, Petzhold, Kopelevich, Jaffe, Mobley, and Holst] that increase understanding of ocean optics and provide a means to not only minimize the limiting optical parameters but also exploit the physics of the underwater environment. Even with years of experience and research available to the scientific community, there is not yet a world-wide database of parametric ocean optical properties available [Smith \& Baker]. In fact, the data most often used for optical modeling, comparison and system design is from Petzhold, which encompass only three different locations from experiments over 30 years ago [Petzhold]. This lack of an ocean-wide database and the thirst for increased documentation of the ocean depths makes the understanding of the root behavior of light in water paramount to design success.

The advent of color remote sensing of the ocean surface, by satellites and aircraft, led to a boom in the desire to study the optical properties of the ocean [Maffione]. By effortlessly viewing the ocean color, oceanographers could infer the organic and inorganic content of the entire world's oceans with a single snapshot from space (see Figure 1). The issue still remained, however, as to how to interpret the different hues and the data represented. Understanding differing colors based on organic and inorganic particles required understanding of the optical properties of the organisms which in turn could provide a step further to understanding the overall optical properties of the ocean. 


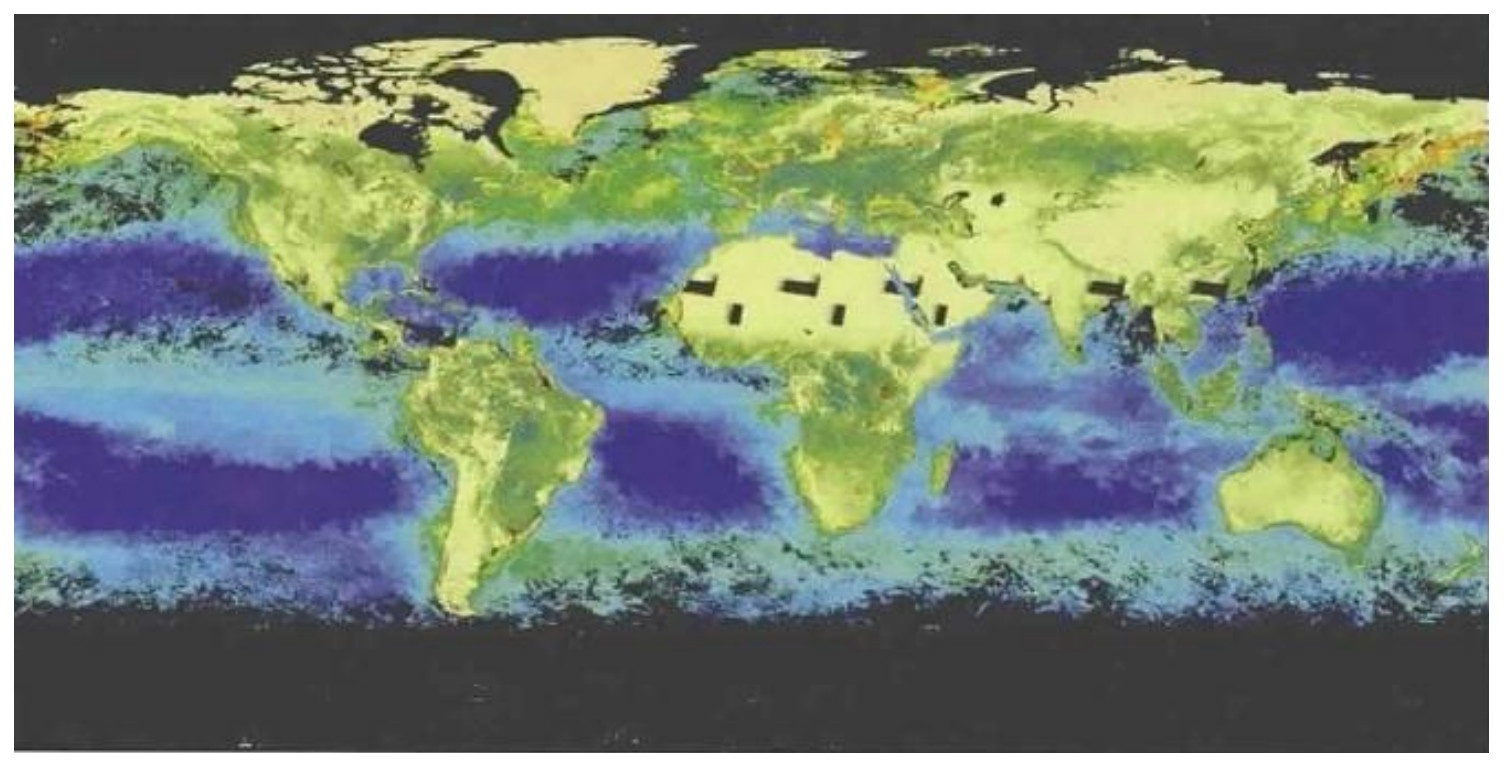

Figure 1- Composite global ocean (and land) color image the SeaWiFS satellite sensor based on the monthly average for May 2001. The advent of color remote sensing of the ocean surface, by satellites and aircraft, led to a boom in the desire to study the optical properties of the ocean (from Maffione).

Unfortunately, to understand the optical properties of the organisms, scientists had to solve the problems concerned with the interaction of light in the ocean. These new technologies led to new concepts, which in turn raised new questions, a reoccurring theme in oceanographic research. Now not only did the optics of the oceans affect underwater imaging, it affected the overall understanding of the entire ecosystem. Again the importance of our understanding of the basic make-up of the ocean optical environment was reiterated.

The optical properties of seawater can be divided into two separate yet related parts; the inherent optical properties and the apparent optical properties. 


\subsubsection{Inherent Optical Properties}

An optical property can be considered inherent, if at a given point in the medium, the property value does not change (invariant) to changes in the light field [Smith \& Baker]. In other words, an inherent optical property (IOP) is controlled only by the seawater, no matter the type or design of the illumination employed. The inherent optical properties of the ocean, at the specific location of imaging, are very important. It is these interactions of the light with the IOPs and the intensity of the light after such interactions that will determine the functionality and quality of an imaging system output.

The operational values of the IOP depend on the dissolved and suspended material in the water, as well as the electromagnetic properties of the medium itself. The properties are easy to define, but can be difficult to measure, especially in-situ [Mobley 2010]. Specifically, a varying combination of IOPs can create the same environmental optics. The IOP combinations are not unique and those measured in one specific area may not represent the actual optical environment present [Sydor, et al].

Although different publications use varying semantics, the inherent optical environment of seawater can be characterized by the volume scattering function (VSF) $(\beta(\theta))$, the volume absorption coefficient $(a)$ and the volume attenuation coefficient $(c)$ [Owen]. The volume scattering coefficient $(b)$, also known as the integral of the VSF, and the single-scatter albedo $\left(\frac{b}{c}\right)$ can also be used. The 
most important parameters are the spectral ${ }^{1}$ absorption coefficient and the spectral volume scattering function. All other parameters can be derived from these two [Mobley 2010]. In fact, if polarization is not present or neglected, the propagation of light in a homogenous medium can be fully described by the absorption coefficient, scatter coefficient and the VSF [Jaffe 2010]. The relationships and definitions of various IOPs are shown in Figure 2 and Table 1 respectively.

As previously stated, IOPs are very difficult to measure in-situ as they are not unique and in addition, they can vary greatly with depth, even in a relatively homogenous body of water. The temporal variance (from seconds to seasons), limits the ability to infer the IOPs of a body of water in the horizontal plane based on a snap-shot in time. These two limitations make it difficult to develop in-situ measurement devices that can be used to update optical models or cover great ranges of ocean volumes.

Modeling of IOPs is also difficult, as the current models rely on assumptions that limit the practicality of their use, and, in addition, are normally based on naturally radiated light, vice illumination systems, as is the scope of this discussion. Underwater image models are further discussed in section 1.5.

\footnotetext{
${ }^{1}$ Spectral defines the parameter as depended on the wavelength of light and is denoted by a $\lambda$ next to the respective parameter
} 


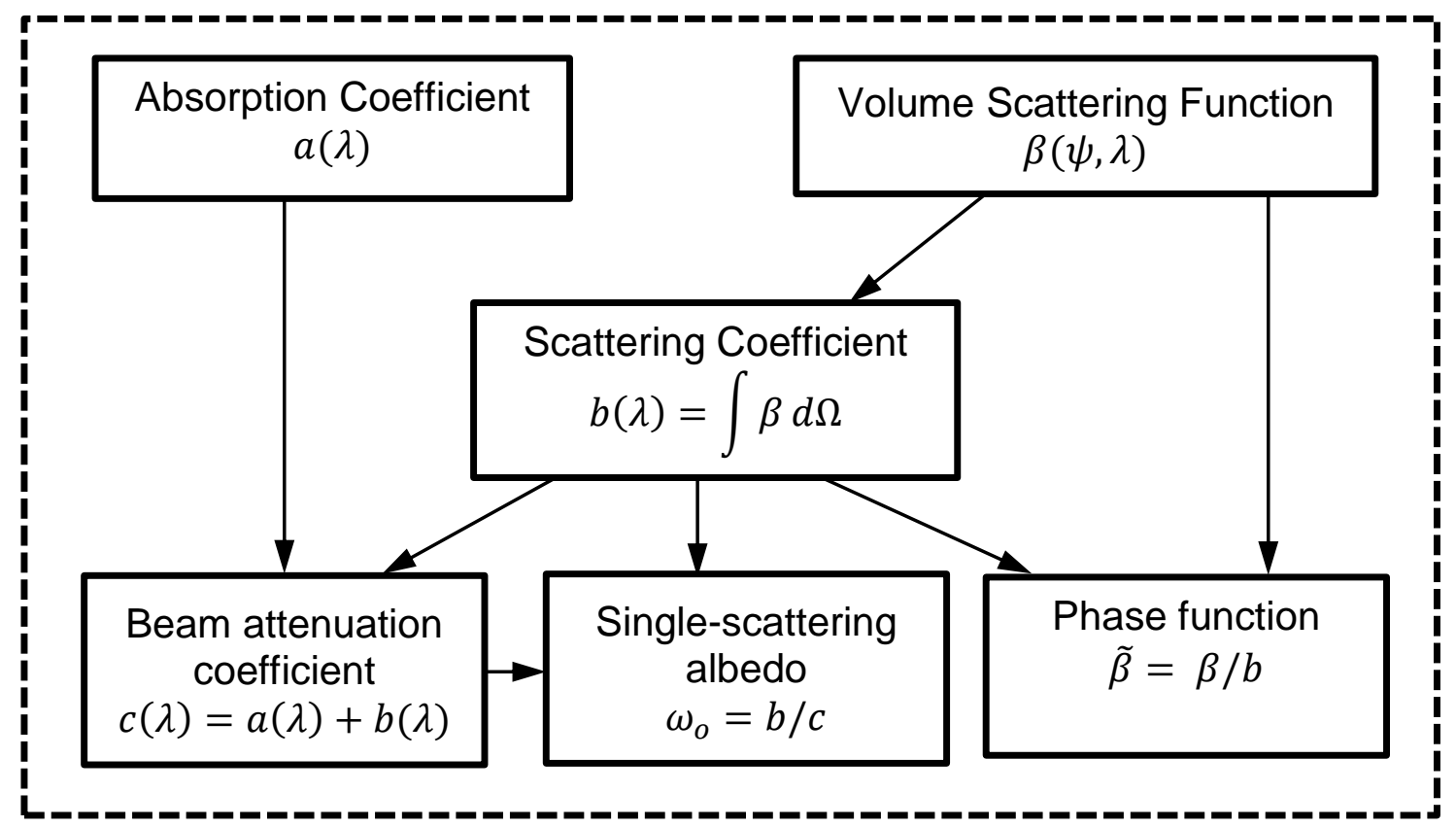

Figure 2 - Inherent optical property relationships [redrawn from Mobley 2010]. Understanding the optical properties of the ocean requires understanding the relationships between each parameter and how they are defined.

\begin{tabular}{lcc}
\hline Quantity & SI Units & Symbol \\
\hline absorption coefficient & $m^{-1}$ & $a$ \\
\hline $\begin{array}{l}\text { scattering coefficient } \\
\text { backward scattering } \\
\begin{array}{l}\text { coefficient } \\
\text { forward scattering }\end{array}\end{array} m^{-1}$ & $b$ \\
coefficient & $m^{-1}$ & $b_{b}$ \\
\hline attenuation coefficient & $m^{-1}$ & $b_{f}$ \\
\hline (real) index of refraction & $m^{-1}$ & $c$ \\
\hline volume scattering function & dimensionless & $\eta$ \\
\hline single-scattering albedo & $m^{-1} s r^{-1}$ & $\beta$ \\
\hline
\end{tabular}

Table 1 - Inherent optical property units (redrawn from Mobley 2010) 


\section{Scattering}

Scattering can be defined as any deviation of a light ray from the incident path. It is a major detractor to image quality, as most images are degraded due to the fact that imaging systems map intensity spatially and cannot recover sufficient optical information in scattering environments [Tyo, et al]. The scattering of the light field in images causes blurring and lack of contrast or a "milky veil" in the final product.

The scattering in a medium is characterized by the volume scattering function (VSF). The VSF is defined as the probability that a ray of light will be deviated by an angle $(\theta)$ from its direction of propagation. The VSF is considered to define the relationship between the IOPs and the apparent optical properties [Kirk]. The integral of the VSF is the volume scattering coefficient. The volume scattering coefficient is defined as the superposition of all scattering events at all angles through the VSF [Schettini \& Corchs]. As previously stated, the VSF and volume scattering coefficient are two of the three IOPs used to characterize the optics of a medium. For general reference, the volume scattering functions for three specific water types (seawater harbor, clear oligotrophic seawater and pure seawater) are shown in Figure 3. 


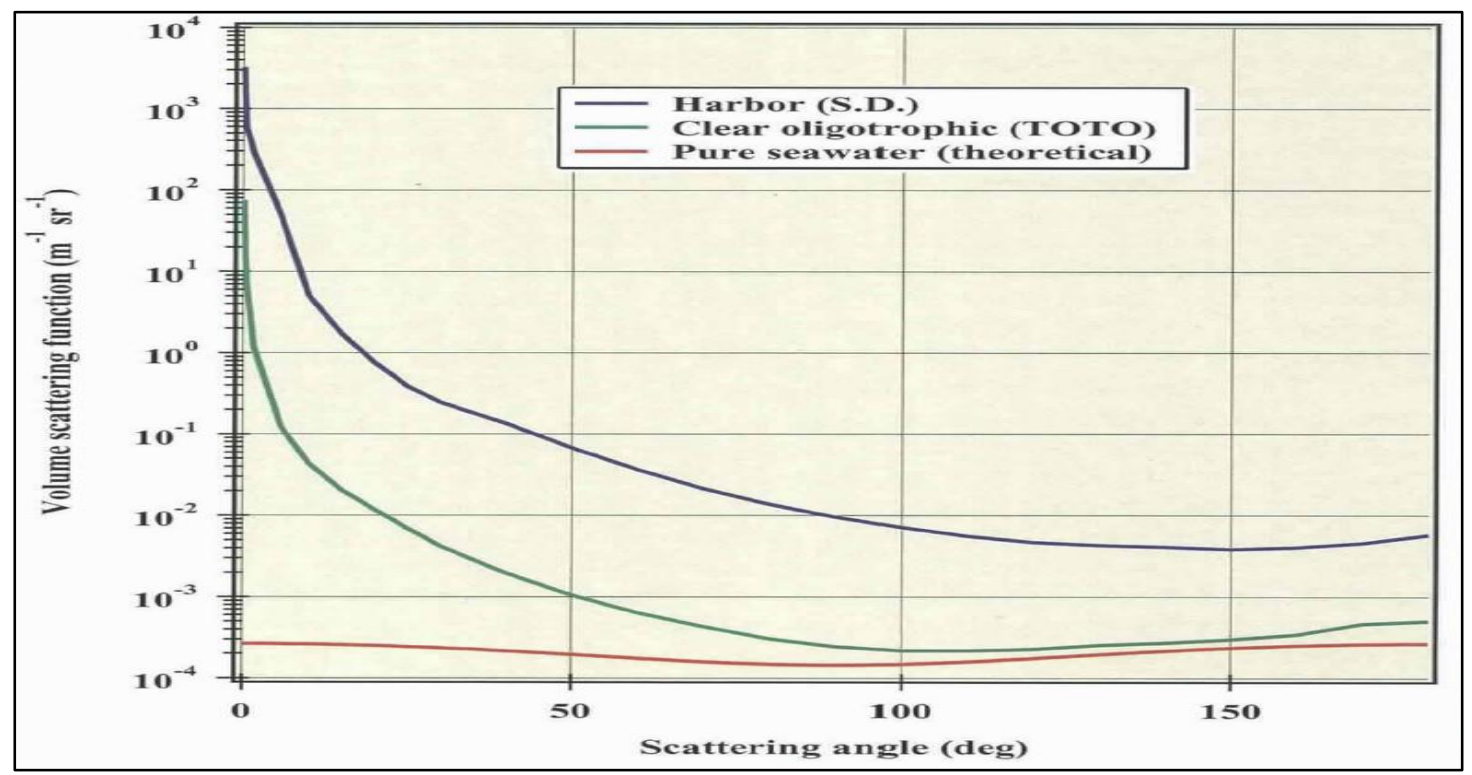

Figure 3 - Volume scattering function for two disparate water bodies; turbid harbor and clear oligotrophic waters (from Maffione 2001). The theoretical VSF for pure water is also included for reference. The increase in scattering based on turbidity is also included for reference. The increase in scattering based on turbidity is shown as an increase in VSF as the seawater bodies clarity decreases

Scattering is composed of two types; forward and backward. Although both types are technically defined as scattering, they are quite different in the actual effect on an illumination field and the resulting image.

In natural waters, the medium of choice for this discussion, the scattering is mainly caused by particles with an index of refraction close to that of seawater and dimensions that are much greater than the wavelength of the incident light. In fact less than $6 \%$ of scattering is at the molecular level [Owen]. 
Forward Scattering

Forward scattering $\left(b_{f}\right)$ is a specular effect that changes the propagation path of a light ray 0 to $\frac{\pi}{2}$ steradians from the incident direction. It causes image blur and can account for the majority of light attenuation during small distance imaging. Schechner and Karpel described the image blurring caused by forward scattering as the convolution of the directly transmitted light $(D)$ and the point spread function parameterized by the distance to the scene $\left(g_{z}\right)$ [Schechner \& Karpel]. The point spread function (PSF) is used to describe the imaging systems response to a point source, or the imaging systems impulse response. The PSF of a system is of great importance because it is easy to measure and can be used to directly derive the volume scattering function of a medium [Jaffe 1995]. In addition, in the absence of absorption, (e.g. small scale experiments, such as the ones presented in this thesis) the point spread function is the definitive environmental characteristic [Jaffe 2010]. 


\section{Backward Scattering}

Backward scattering $\left(b_{b}\right)$ is a specular effect that changes the propagation path of a light ray from $\frac{\pi}{2}$ to $\pi$ steradians from the incident direction. It creates the "milky veil" that is sometimes present in underwater images. Backscatter is similar to the effect of haze, smoke or fog on car headlights, especially when operated in the high beam mode. An example of backscatter contribution to a final image, particularly those observed with limited source receiver separation is reproduced as figure 4 . Source and receiver separation as a scatter mitigation process is discussed further in section

\subsection{1.}

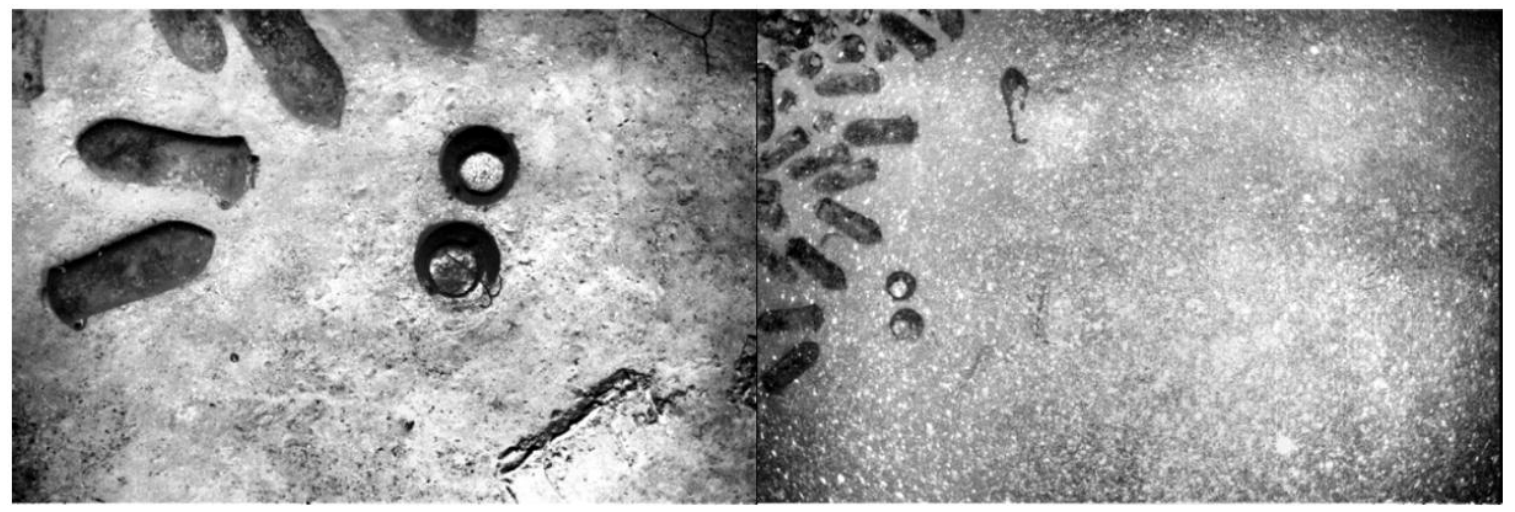

Figure 4 - Backscatter contribution to image quality. Both images were taken by the Jason ROV at differing altitudes; $4 \mathrm{~m}$ for the image on the left and $8 \mathrm{~m}$ for the image on the right. The higher altitude image on the right shows a marked decrease in image quality due to backscatter of the artificial illumination source (from Singh, et al). 


\section{Absorption}

Absorption $(a)$ is the third and final inherent optical property that will be discussed. It is also a specular effect that involves the transformation of photon's energy from one form to another, the latter usually heat. Absorption is a very important concept to consider when designing an imaging system, especially when operating at longer ranges. At the peak transmission wavelengths (between approximately 400 to $500 \mathrm{~nm}$ ), $50 \%$ of attenuation can be contributed to just absorption ${ }^{2}$ [Owen]. It is important to note that absorption (like the other IOPs) is wavelength dependent, but also greatly depends on the organics suspended in the water volume. Red light is normally fully attenuated on $\mathrm{O}(5 \mathrm{~m})$ in open ocean, but has a longer predicted path length in coastal waters where yellow light is the first to be absorbed. General absorption at various depths and differing water types in shown in figure 5 .

\footnotetext{
${ }^{2}$ Of that attenuation, $50 \%$ is due to water (constant), $13 \%$ by particles and $37 \%$ by dissolved by organics [Owen]
} 

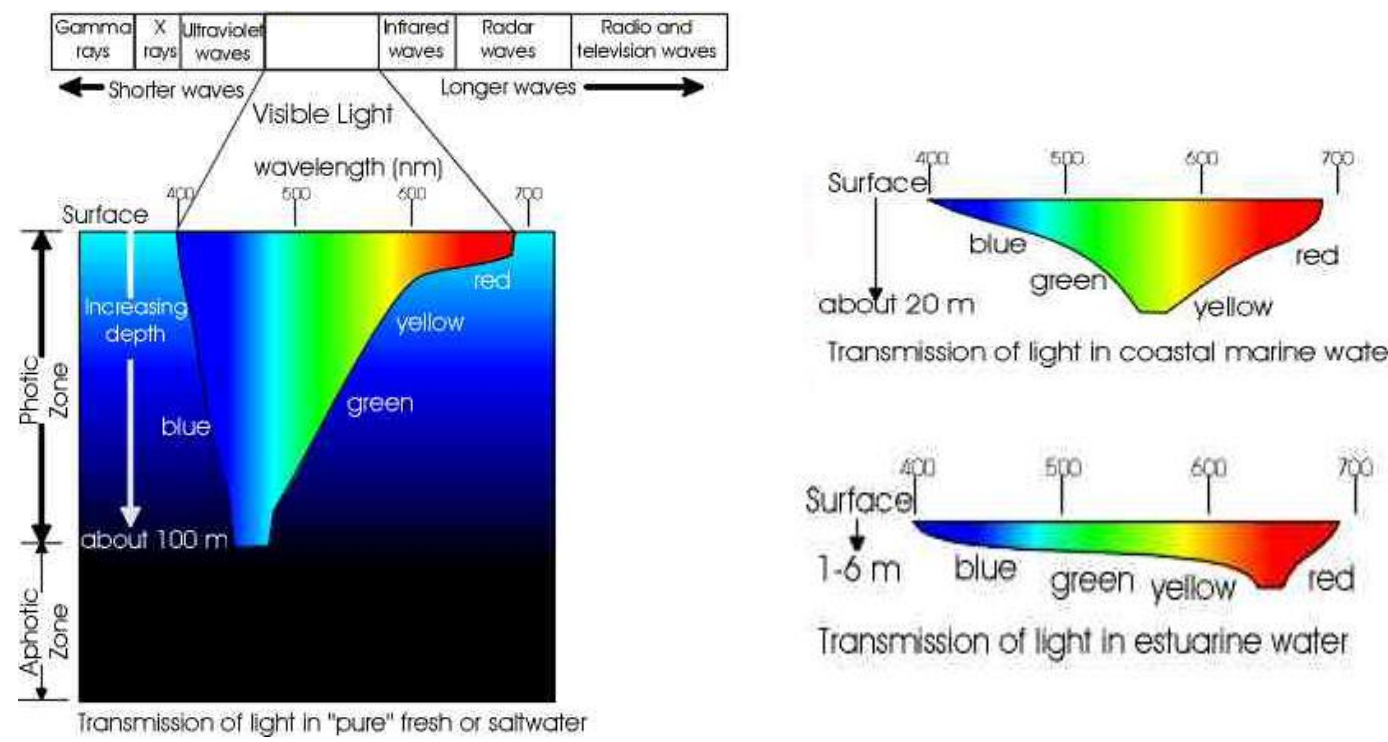

Transmisston of light in estuarine water

Figure 5 - Light absorption in seawater in three disparate water bodies (from http://www.marinebiology.org/oceanography.htm)

\section{$\underline{\text { Attenuation }}$}

Attenuation $(c)$ is a term used to define the combined effects of scattering and absorption with $c=a+\left(b_{f}+b_{b}\right)$. The attenuation coefficient is used as a basis for quantifying light propagation in terms of attenuation lengths. An attenuation length is defined as $\frac{1}{c}$, or the distance one would expect light to propagate until completely absorbed and / or scattered. Some typical considerations for attenuation lengths are that in clear water light is completely attenuated in 20 meters, coastal water in less than 5 meters, and turbid harbor waters in under 3 meters [Bazeille, et al],[Schettini \& Corchs].

Light attenuation can be described in the terms of the exponential decay of object radiance and term the resulting light as directly transmitted light $(D)$, 
$D=L_{\text {object }} e^{c z}$. With $L_{\text {object }}$ defined as the object radiance one would sense with no scattering or absorption along the line of sight, $c$ as the attenuation coefficient and $z$ the distance to the object plane [Schechner \& Karpel]. I modified the original annotation for the attenuation coefficient as originally proposed in the previously cited paper to maintain consistency among this thesis and numerous publications.

To fully understand and define the inherent optical properties of seawater, one must understand the geometry of the pieces that make up the whole. Upon discussion and general understanding of the effects, figure 6 shows the interaction within a volume of participating media. As described by Mobley, $\Phi_{i}(\lambda)=\Phi_{a}(\lambda)+\Phi_{s}(\lambda)+\Phi_{t}(\lambda)$, with $\Phi$ as radiant power in $\mathrm{W} \mathrm{nm}^{-1}$ and subscripts $i$ as incident, a as absorbed, $s$ as scattered and $t$ as transmitted. As the incident light enters a volume of water (participating medium), some part of the incident illumination radiant power is absorbed in the medium, and the remainder is either scattered at an angle $(\theta)$ or transmitted with no change in direction from original path. 


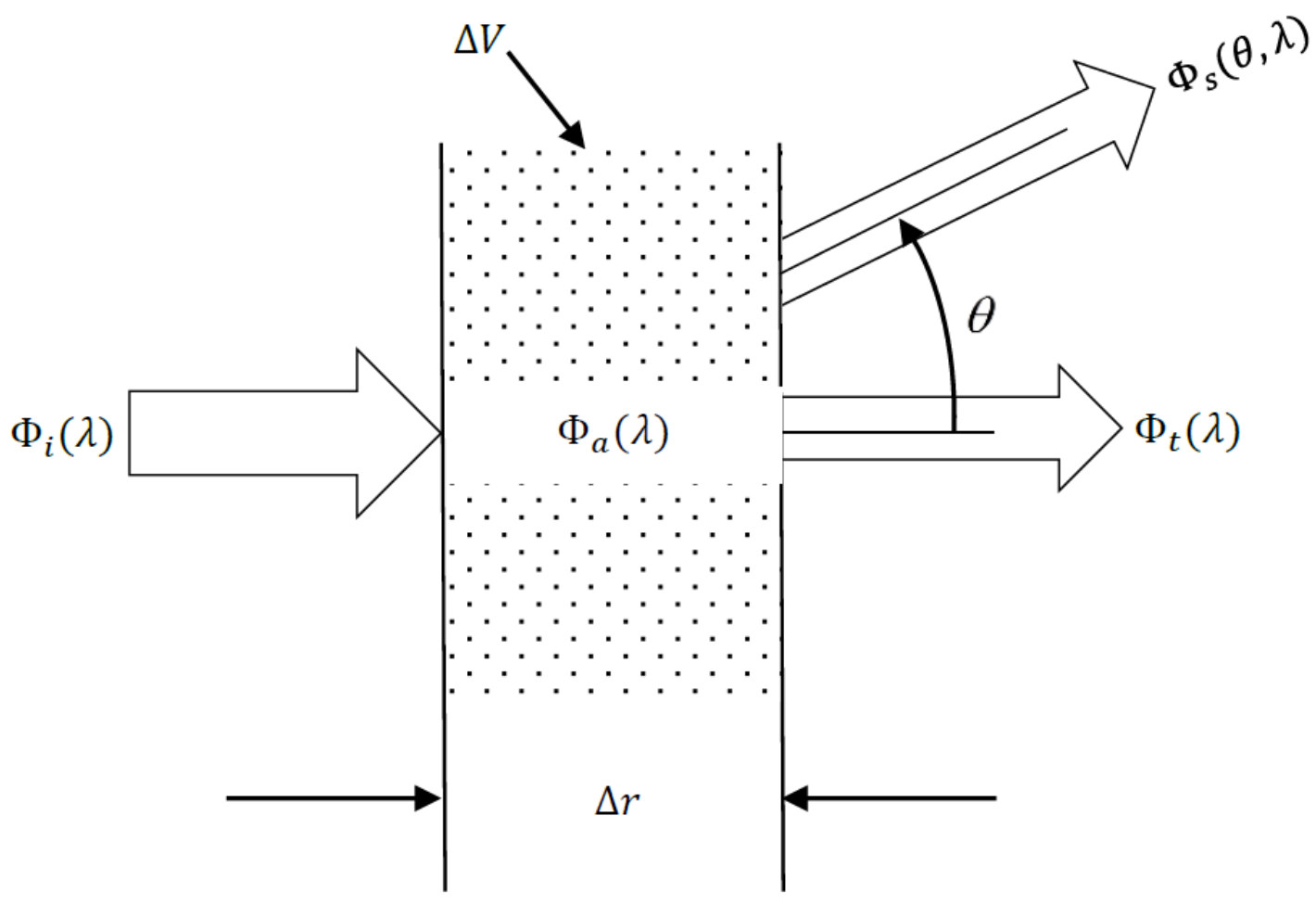

Figure 6 - Inherent optical property geometry (redrawn from Mobley 2010). To fully understand and define the inherent optical properties of seawater, one must understand the geometry of the pieces that make up the whole. Upon discussion and general understanding of the effects this figure illustrates the interaction of inherent optical properties within a volume of participating media. 


\subsubsection{Apparent Optical Properties}

There are also optical properties in seawater that are dependent on both the local IOP and the illumination field incident on the area of interest. An apparent optical property $(\mathrm{AOP})$ can be defined as a property whose operational value at a given point in a medium is dependent upon the radiance distribution at that point [Smith \& Baker]. Although not a significant topic of discussion or experimentation for dynamic lighting design, AOPs are important in radiative transfer theory. Some commonly used AOPs are listed as Table 2

\begin{tabular}{lcc}
\hline Quantity & SI Units & Symbol \\
\hline distribution function & dimensionless & $D$ \\
\hline average cosine of light field & dimensionless & $\bar{\mu}$ \\
of downwelling light & dimensionless & $\bar{\mu}_{d}$ \\
of upwelling light & dimensionless & $\bar{\mu}_{u}$ \\
\hline irradiance reflection & dimensionless & $R$ \\
\hline remote sensing reflectance & $s r^{-1}$ & $R_{r s}$ \\
\hline $\begin{array}{l}\text { (vertical) diffuse attenuation coefficients (K-functions) } \\
\text { of radiance } L(z ;, \theta, \phi)\end{array}$ & $m^{-1}$ & $K(\theta, \phi)$ \\
of downward irradiance $E_{d}(z)$ & $m^{-1}$ & $K_{d}$ \\
of upward irradiance $E_{u}(z)$ & $m^{-1}$ & $K_{u}$ \\
of downward scalar irradiance $E_{o d}(z)$ & $m^{-1}$ & $K_{o u}$ \\
of upward scalar irradiance $E_{o u}(z)$ & $m^{-1}$ & $K_{o}$ \\
of total scalar irradiance $E_{o}(z)$ & $m^{-1}$ & $K_{P A R}$ \\
of $P A R(z)$ & $m^{-1}$ & \\
\hline
\end{tabular}

Table 2 - Apparent optical property units (redrawn from Mobley 2004) 


\subsection{Different Ocean Environments}

One of the main and lasting challenges of understanding the optical properties of seawater is that they continually change both temporally and spatially. The inherent optical properties in the clear ocean waters of the Caribbean are completely different than the harbor waters or even coastal waters off of the United States. In addition, each change in the inherent optical properties causes a change in the apparent optical properties, which can be further complicated by a change in the incident light field by something as simple as a passing cloud.

To further complicate the environment, and to continue the trend of more knowledge begets more questions, some scientists have provided scientific hypothesis of additional optics changing variables in seawater. Two of the most recent were the additional effects of turbulence [Hou, et al 2011] and salinity [Zhang, et al] on the optical properties of seawater. These advances continue to define the operational and design space, but also limit our ability to design a "one-stop shop" system for underwater imaging. 


\subsection{Underwater Imaging Models}

The lack of an overarching IOP database and limited ability to measure in-situ demands that researchers and scientists use various underwater imaging models to describe the total underwater optics environment. There are many different models to choose from, including: a model with turbulence affects [Hou, et al 2011], a model with seawater density and salinity concentration affects [Zhang, et al] and Monte-Carlo modeling [Jaffe 1995 and Mobley 2004]. The latter is especially useful and used (at least initially) by many researchers to find and compare numerical solutions to radiance transfer equations. These equations are the bridge between the inherent and apparent optical properties of the underwater environment and understanding the results provided by these models will be crucial in defining the operating and design boundaries of the dynamic lighting system presented in this thesis. 


\section{CHAPTER 2: IMAGING IN TURBID ENVIRONMENTS}

\subsection{Current Mitigation Techniques}

The movement to understand and conquer the challenging ocean optical environment is well documented by numerous researchers. Many mitigation techniques are currently utilized to improve image quality and extend imaging range.

An interesting point to note, is that "easy" and commonly used computer vision techniques, such as high pass filtering (sharpening), low pass filtering (smoothing) and histogram equalization (contrast improvement) are spatially invariant methods [Schechner \& Karpel]. As discussed in previous sections, image degradation in seawater is caused by spatially variant parameters and is therefore is not easily remedied by classic computer vision techniques.

Some underwater image degradation minimization techniques are briefly discussed below and will concentrate on those methods specifically designed for use in scattering media.

\subsubsection{Source and receiver separation (SRS)}

Physically separating the source and receiver in an imaging system is a simple and effective way to decrease scatter interactions with the incident light from the illumination source. This method limits the size of the area that source light and the participating medium interact, thereby decreasing the scatter interaction 
volume and reducing image degradation. An everyday example would be the placement of fog lights in a modern automobile. The increased separation of the fog lights and driver's field of view, as compared to the normal headlights, are what allows increased visibility during periods of fog or haze. A visual representation of the scattering interaction volume is shown in figure 7 and is defined as the volume that includes the overlapping field of view of the sensor and field of illumination of the source contained in a participating medium. A more in depth definition is that the scatter interaction volume is the volume of intersection between the fields of view of the camera pixel that observes the surface point and the source element that illuminates it [Nayar, et al].
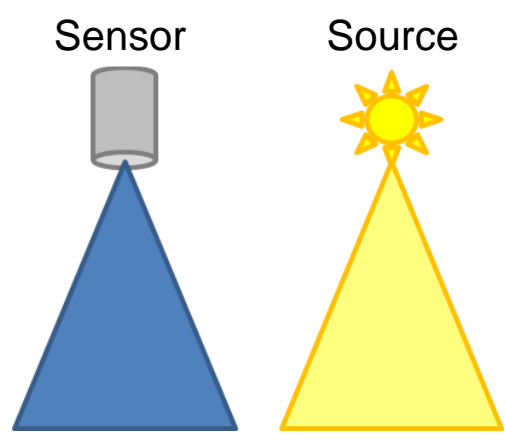

(a)

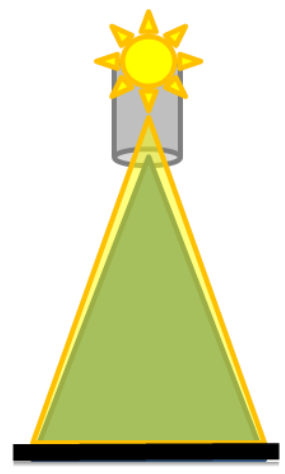

(b)

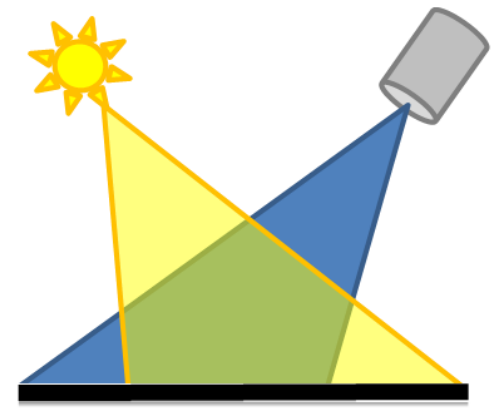

(c)

Figure 7 - Scatter interaction volume differences based on source / sensor placement. (a) The sensor field of view shown in blue and the source field of illumination shown in yellow. (b) With sensor and source co-located, the scatter interaction volume is the largest. (c) By using sensor / source separation, the scatter illumination volume size decreases. 
SRS is easy to implement, but does have the disadvantage of increasing system size and at larger separation distances can cause image artifacts such as shadowing (see figure 8 ). Not all imaging platforms have the ability to provide adequate source and receiver separation and there is always a trade-off between system size, power available and imaging coverage. The lighting characteristics of different imaging platforms are provided for comparison in table 3. In systems intended to be used at long ranges and / or very turbid water, multiple scattering can decrease the effectiveness of the separation [Mullen, et al]. Optimal separation for flood-lighting was experimentally determined as $40 \mathrm{~cm}$ [Gupta, et al].

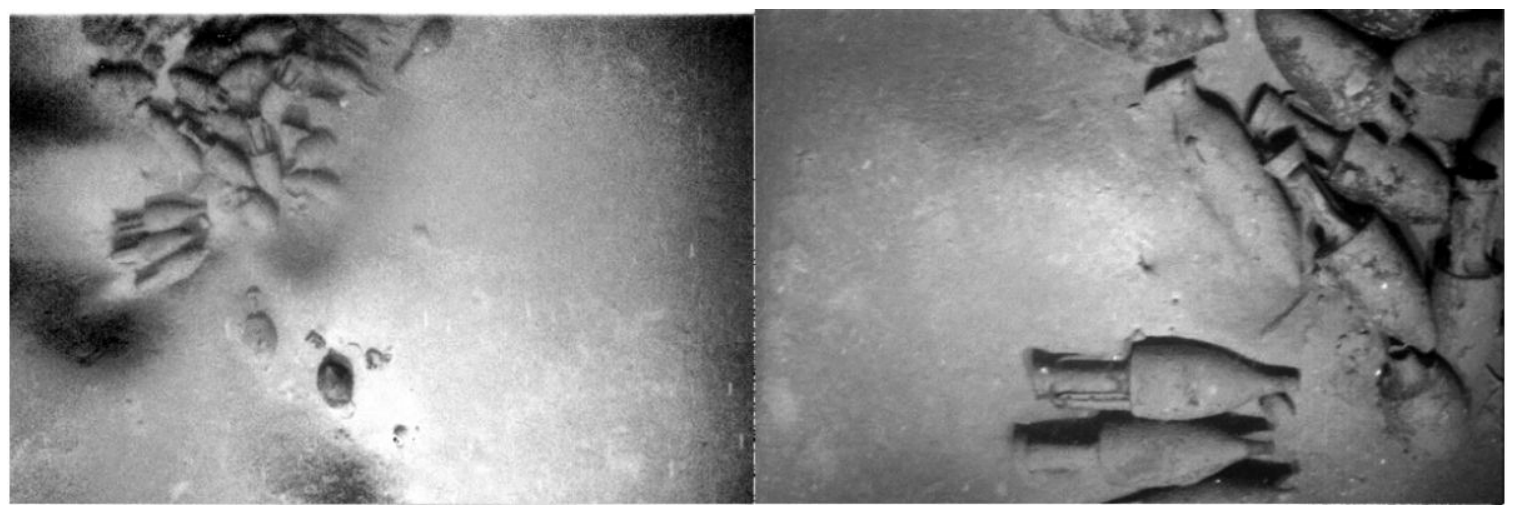

Figure 8 - Shadowing artifacts caused by large source / receiver separation. The image on the left shows clear shadowing artifacts in the bottom topography caused by a large SRS, while the image on the right (of the same scene) with a smaller SRS shows an artifact free bottom (from Singh, et al). 


\begin{tabular}{lllll}
\hline Platform & $\begin{array}{l}\text { Source / Rcvr } \\
\text { Separation }\end{array}$ & Power & $\begin{array}{l}\text { Relative } \\
\text { Coverage }\end{array}$ & Limitations \\
\hline NR-1 Submarine & $\begin{array}{l}\text { Large O(several } \\
\text { meters) }\end{array}$ & $\begin{array}{l}\text { Essentially } \\
\text { Unlimited }(10 \mathrm{~kW})\end{array}$ & $\mathrm{O}(10 \mathrm{~m} \times 10 \mathrm{~m})$ & $\begin{array}{l}\text { Limited by } \\
\text { scattering }\end{array}$ \\
\hline DSV Alvin & Large O(meters) & Limited O $(1 \mathrm{~kW})$ & $\mathrm{O}(5 \mathrm{~m} \times 5 \mathrm{~m})$ & $\begin{array}{l}\text { Limited power } \\
\text { with good SRS }\end{array}$ \\
\hline ROV Jason & Medium & $\begin{array}{l}\text { Essentially } \\
\text { Unlimited } \\
\text { O(meters })\end{array}$ & $\mathrm{O}(5 \mathrm{~m} \times 5 \mathrm{~m})$ & $\begin{array}{l}\text { Backscatter } \\
\text { limited }\end{array}$ \\
& O(10kW) & $\mathrm{O}(1 \mathrm{~m} \times 1 \mathrm{~m})$ & $\begin{array}{l}\text { Non-uniform } \\
\text { lighting / low } \\
\text { overlap }\end{array}$ \\
\hline AUV SeaBed & Limited O $(\mathrm{m})$ & $\begin{array}{l}\text { Very limited } \\
\mathrm{O}(0.1 \mathrm{~kW})\end{array}$ & $\mathrm{O})$ & $\begin{array}{l}\text { Environmental } \\
\text { and training } \\
\text { considerations }\end{array}$ \\
\hline Diver & Very limited & $\begin{array}{l}\text { Very limited } \\
\mathrm{O}(\mathrm{W})\end{array}$ & $\mathrm{O}(200 \mathrm{~cm} \times$ \\
& $\mathrm{O}(\mathrm{cm})$ & $200 \mathrm{~cm})$ & \\
\hline
\end{tabular}

Table 3 - Lighting characteristics for various imaging platforms (redrawn from Singh, et al).

\subsubsection{Spatial Filtering}

Spatial filtering is used to remove unwanted light intensity variations across a light beam using a spatial filter. It is primarily for laser imaging applications and utilizes a collimation device and a pinhole to physically remove spatial noise.

The term spatial filtering is mentioned in other publications to indicate any system that reduces the interaction volume between a participating medium and an illumination source [Mullen, et al, Kulp, et al and Strand].

\subsubsection{Range Gating}

Range gate techniques are typically used in green-laser applications [Kocak, et al] and improve image contrast by timing illumination and image capture such that most of the backscattered light (arriving earlier than target light) is rejected. The difference in the time of arrival to the receiver for backscatter and a target 
return are shown in Figure 9. Both the timing of the returns and their corresponding shape can be utilized to minimize the capture of backscattered light. Specific methods have shown visibility up to 5 attenuation lengths [Jaffe 2001] but the systems have the limitations of complexity and increased expense [Walker, et al].

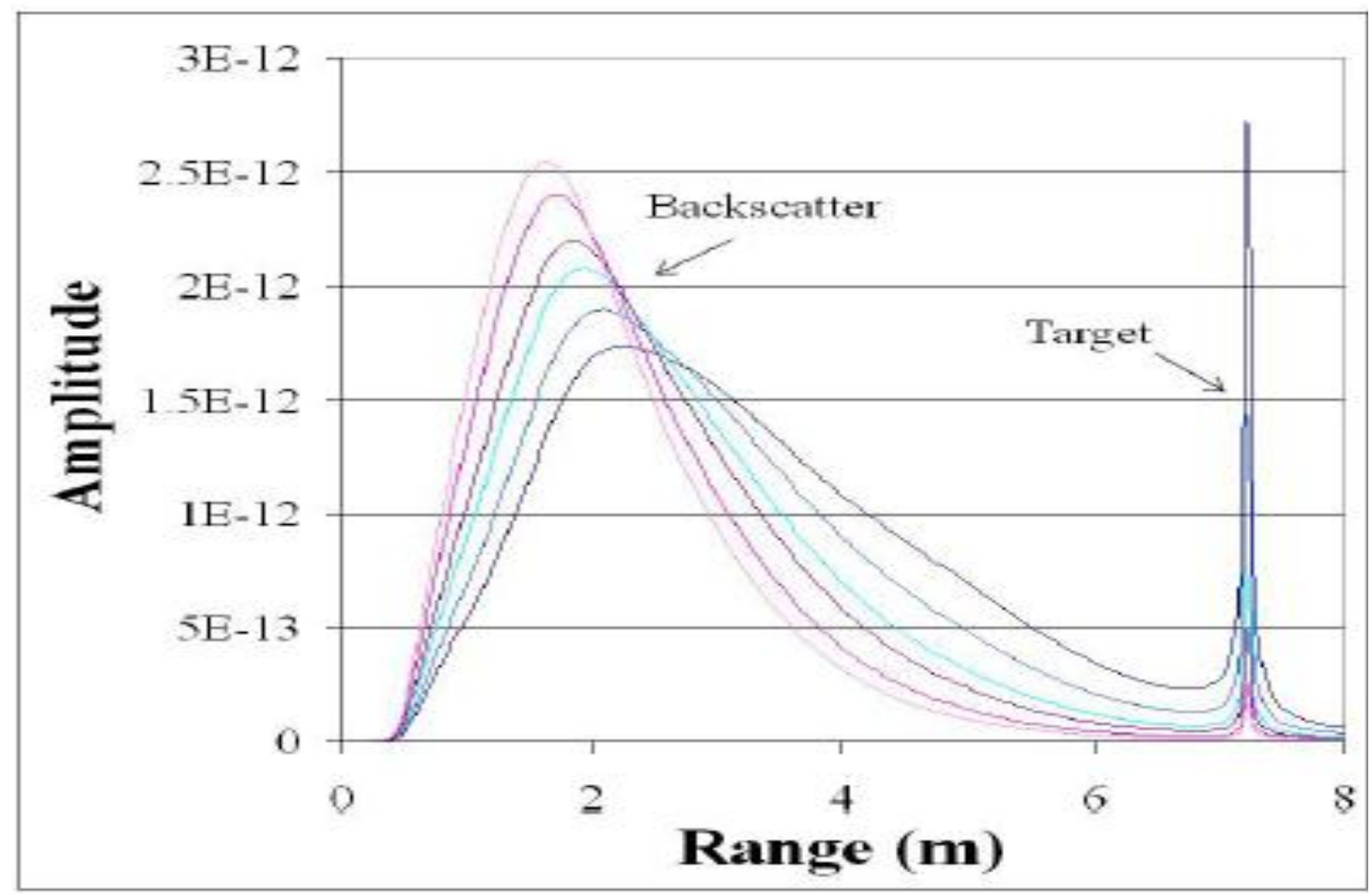

Figure 9 - Backscatter and target-reflected signals showing differences in the time of arrival and shape of returns. By timing the camera to receive after the backscatter peak has passed, one can remove the majority of backscatter effects while maintaining the scene information (from Mullen, et al).

\subsubsection{Synchronous Scanning}

Laser line scan and spatial discrimination techniques use a highly collimated beam of laser light to scan perpendicular to platform movement. The system imaging sensor is synchronously scanned with the laser to present a narrow field 
of view to light returns [Jaffe 2001]. These methods have shown up to 6 attenuation lengths of visibility in turbid seawater [Kocak, et al].

\subsubsection{Polarization}

Similar to range gating and in general, polarization techniques take advantage of the different polarization properties of the target and backscattered light returns [Mullen, et al]. This method is based on the assumption that linearly polarized light will be depolarized by a target but the polarization will remain in the light encountering any backscattering. A receiver polarizing filter will then reject the backscattered light and retain the majority of the target information.

Polarization techniques can be roughly divided into two types, passive and active. Passive techniques use ambient or natural lighting, while active techniques used linearly or circularly polarized artificial illumination.

\section{$\underline{\text { Passive }}$}

One passive technique uses ambient light from the sun or a cloudy sky [Chang, et al]. The results showed that ambient illumination can be used in turbid waters and subsequent imaging distances and quality improved by separation of the polarized backscattered light from the almost completely un-polarized target returns. It was also shown that a significant amount of polarization is the result of single scattering by small particles. The technique was successful for relatively small scatterers (Rayleigh particles) and showed less improvement with larger occluders (Mie particles). 
Another passive technique was concerned with the assumption that in naturally illuminated scenes, polarization is the primary degradation factor to image quality [Schechner \& Karpel]. In this technique, the researchers used multiple images, each at differing states of polarization to improve the target images by postprocessing. Results showed that visibility ranges roughly doubled.

\section{$\underline{\text { Active }}$}

One active technique, based on the visual system of a specific fish, is the polarization difference imaging technique [Tyo, et al]. This method used two orthogonal polarized light sources and the affine transformed difference of the two sources to improve, in this experiment, target detection. A similar image difference technique [Walker, et al] used the difference between a co-polarized image (polarization of the image was the same as polarization of the illumination) and a portion of the opposite-polarized image. The optimal opposite-polarized image portion was experimentally determined as $\sim 13$ percent.

Circular polarization was demonstrated to improve imaging systems by utilizing a circular polarizer and circular analyzer in concert to minimize the contribution of single-scatter polarization affects [Gilbert \& Pernicka]. This technique, with a minimally separated source and receiver, improved target acquisition to eight attenuation lengths as opposed to just four in similar conditions and with no polarization methods imposed.

The final active polarization technique discussed is polarized light-striping [Gupta, et al]. This method combined structured light concepts and polarization 
techniques to reduce the scattering interaction volume while further improving imaging by previously stated active polarization techniques.

\subsubsection{Modulated waveform illumination}

Modulated waveform illumination used coded modulated laser pulses to take advantage of the difference in the shape (rise time, fall time and pulse width) between scattered light and target reflected light, as illustrated in figure 9. This method uses high-frequency modulation coupled with short optical pulses to minimize scatter in both the forward and backward direction [Mullen, et al].

\subsubsection{High Frequency Illumination}

The high frequency illumination technique [Nayar, et al] used a binary pattern (checkerboard) projected onto a scene in order to allow post-process separation of global and direct illumination components. The theory suggested that only two images were required to conduct the separation, but the experiments were conducted using a large number of images to correct camera and illumination limitations. This method was not a pure image enhancement technique, but can be used to quickly remove the shadowy veil caused by backscatter in a turbid underwater image.

\subsubsection{Pre-processing}

One interesting pre-processing filter approach was published [Bazeille, et al] that used several computer vision techniques in succession for image restoration. It is an important algorithm to note, as it incorporated several novel restoration 
techniques that corrected for non-uniform illumination, improved signal-to-noise ratio, and improved contrast and mediated colors. This method could be used in concert with the previously discussed mitigation techniques to further improve image quality.

\subsubsection{Confocal imaging [Levoy \& Singh]}

Confocal imaging in turbid media was based on the principle of confocal microscopy where optical apertures were used to minimize the contribution of targets not on the focal plane. In this imaging technique the optical apertures were replaced by synthetic apertures formed by arrays of projectors and cameras. This technique could then be used to minimize the contribution of scattered light (similar to reducing off focal plane target contributions in confocal microscopy) in a turbid water environment.

The research in this thesis was based on the confocal imaging system design provided by Levoy and Singh. The system provided in the published paper differs from the current design point of being field deployable, as the Levoy and Singh's system was composed of multiple LCD projectors. Since 2009, portable DLP devices and handheld projectors have improved dramatically, in both decreased size and reduced cost and these advances have allowed the original confocal imaging research to be further refined in my research to yield a better field deployable system. 


\subsection{Image Quality}

One topic that has not been discussed thus far is a common quantitative metric for image quality. Although many of the previous mitigations methods included a direct mention of attenuation length improvement, the actual basis for comparison was either not mentioned or varied from technique to technique. An imaging technique can be considered "extended range" if it operates at greater than 3 attenuation lengths [Jaffe 2010]. An issue with attenuation length, as previously discussed, is that it is not unique. The attenuation coefficient is made up of both coefficients for scattering and absorption and different combinations can result in identical attenuation lengths. Several different environments, with vastly differing optical properties, could therefore have similar attenuation lengths and skew experimental data.

A common measureable metric for image quality is not available and is the subject of many publications and research endeavors. Image quality methods are broken down into subjective and objective methods. Objective methods are based on quantitative data, while subjective methods rely on individual physical interpretation of an image. As one would imagine, subjective methods rely on the vision, perception and sometimes mood of a particular subject, hence the need for a robust and easily implemented objective method of image quality. The subjective and objective methods can be further broken down into full, partial (or reduced) and no-reference classes, which are determined by how, and if, the tested image is compared with a reference image. Full reference metrics 
assumes that a complete set of information is known about the original image, partial assumes that some information is known and no-reference means that the original image is not available or not used [Ouni, et al]. The following two tables (tables 4 and 5) provide a brief background into several methods of image quality assessment.

\begin{tabular}{l|l|l|l}
\hline $\begin{array}{l}\text { Image Quality } \\
\text { Assessment } \\
\text { Techniques }\end{array}$ & Class & Discussion & Reference \\
\hline $\begin{array}{l}\text { Double stimulus } \\
\text { continuous quality } \\
\text { scale (DSCQS) }\end{array}$ & Full & $\begin{array}{l}\text { Test and reference images are compared and } \\
\text { rated in random order of pairs. Test or } \\
\text { reference images are shown first and the } \\
\text { method is conducted twice. Rating scale is } \\
\text { from bad to excellent (0 to 100). Should be } \\
\text { used for test and reference images that are of } \\
\text { similar. }\end{array}$ & Miras \\
\hline $\begin{array}{l}\text { Double stimulus } \\
\text { impairment scale } \\
\text { (DSIS) }\end{array}$ & Full & $\begin{array}{l}\text { Similar to DSCQS, but test image is always } \\
\text { presented prior to reference and pairs are only } \\
\text { shown once. Rated from very annoying to } \\
\text { imperceptible (0 to 5). }\end{array}$ & Miras \\
\hline $\begin{array}{l}\text { Single stimulus } \\
\text { continuous quality } \\
\text { scale (SSCQS) }\end{array}$ & No & $\begin{array}{l}\text { Subject watches a long series of test images } \\
\text { (no reference shown) and continually adjusts a } \\
\text { sliding DSCQS scale. }\end{array}$ & Miras \\
\hline $\begin{array}{l}\text { Single stimulus } \\
\text { impairment scale } \\
\text { (SSIS) }\end{array}$ & No & $\begin{array}{l}\text { Similar to SSCQS, but sliding scale is from 0 to } \\
5\end{array}$ & Ouni, et al \\
\hline \begin{tabular}{l} 
Comparison \\
\hline
\end{tabular} & Partial & $\begin{array}{l}\text { Pairs of images are compared rated better or } \\
\text { worse. }\end{array}$ & Ouni, et al \\
\hline
\end{tabular}

Table 4 - Subjective image quality assessment techniques 


\begin{tabular}{|c|c|c|c|}
\hline $\begin{array}{l}\text { Image Quality } \\
\text { Assessment } \\
\text { Techniques }\end{array}$ & Class & Discussion & Reference \\
\hline Peak SNR & $\begin{array}{l}\text { Full or } \\
\text { Partial }\end{array}$ & $\begin{array}{l}\text { Measures mean squared error between } \\
\text { reference and test images }\end{array}$ & Miras \\
\hline Mathematical models & Full & $\begin{array}{l}\text { Measures the statistical differences between } \\
\text { the reference and test images }\end{array}$ & Ouni, et al \\
\hline $\begin{array}{l}\text { Human visual system } \\
\text { models }\end{array}$ & Any & $\begin{array}{l}\text { Hybrid method encompassing both objective } \\
\text { and subjective measurements that attempts to } \\
\text { simulate the human visual process to } \\
\text { determine image quality. }\end{array}$ & $\begin{array}{l}\text { Ouni, et al } \\
\text { Larson \& } \\
\text { Chandler }\end{array}$ \\
\hline $\begin{array}{l}\text { Motion vector } \\
\text { histogram }\end{array}$ & Partial & $\begin{array}{l}\text { Extracts motion vectors from reference and } \\
\text { target images and compares the resulting } \\
\text { histograms. }\end{array}$ & Han, et al \\
\hline Weighted GSA & No & $\begin{array}{l}\text { Measures the sharpness of an image using the } \\
\text { weighted grey scale angle (GSA) of detected } \\
\text { edges. }\end{array}$ & $\begin{array}{l}\text { Hou \& } \\
\text { Weidemann } \\
2007\end{array}$ \\
\hline $\begin{array}{l}\text { Blur radius and visual } \\
\text { blockiness }\end{array}$ & No & $\begin{array}{l}\text { Measures the blur radius based on edge } \\
\text { differences and magnitudes, as well as visual } \\
\text { blockiness based on brightness differences. }\end{array}$ & Jeong, et al \\
\hline $\begin{array}{l}\text { Singular value } \\
\text { decomposition }\end{array}$ & No & $\begin{array}{l}\text { This method was designed to concentrate on } \\
\text { loss of image structure vice distortion of pixel } \\
\text { intensity values. Use both singular vectors and } \\
\text { singular values as weighted features for image } \\
\text { distortion information. }\end{array}$ & Wang, et al \\
\hline $\begin{array}{l}\text { Edge detection } \\
\text { robustness criteria }\end{array}$ & Full & $\begin{array}{l}\text { Compares the gradient magnitude histogram of } \\
\text { a target image to the reference image to } \\
\text { determine quality. }\end{array}$ & $\begin{array}{l}\text { Bazeille, et } \\
\text { al }\end{array}$ \\
\hline $\begin{array}{l}\text { Wavelet } \\
\text { decomposition }\end{array}$ & & $\begin{array}{l}\text { Sharpness of each edge of the target image is } \\
\text { determined by regression (after wavelet } \\
\text { decomposition). The regression is used to } \\
\text { determine the GSA of edge pixels versus } \\
\text { location. This method is used to automate an } \\
\text { image restoration algorithm }\end{array}$ & $\begin{array}{l}\text { Hou, et al } \\
2007\end{array}$ \\
\hline $\begin{array}{l}\text { Modulation transfer } \\
\text { function (MTF) }\end{array}$ & No & $\begin{array}{l}\text { By determining an imaging system MTF, one } \\
\text { can determine the perceived quality of } \\
\text { resulting images. The higher the MTF the } \\
\text { better the quality }\end{array}$ & Holst \\
\hline Contourlet transform & Full & $\begin{array}{l}\text { Uses the contourlet transform, a pyramidal } \\
\text { directional filter bank, to build the intrinsic } \\
\text { geometric features of a target and reference } \\
\text { image for comparison based on the multi- } \\
\text { directional difference model. }\end{array}$ & Liu \& Yang \\
\hline
\end{tabular}

Table 5 - Subjective image quality assessment techniques 


\section{CHAPTER 3: THE DYNAMIC LIGHTING SYSTEM}

\subsection{Environmental Design Constraints}

What is clear about the underwater environment is that it presents multiple challenges to the design and operation of an imaging system. With a firm grasp of inherent and apparent optical properties, a defined area of operation that provides some stability in terms of conditions and an adequate model to map the road to design, one still has to contend with the additional challenges that operating in seawater entail.

These additional constraints include, but are not limited to: extreme pressures at depth, oxidizing properties of seawater and the power limitations for autonomous vehicle mating.

\subsubsection{Pressure}

With the design of any system for exploration under the ocean, one must consider the immense pressures at depth. Oceanographic research spans the range of depths, from shallow ambient light studies in an ocean tidal pool, to the study of the deepest hydrothermal vents several thousand meters below the ocean surface. This range requires system design to encompass the pressures at the maximum depths expected in the lifecycle of a vehicle. During the search for Air France flight 447 off of the coast of Brazil, for example, wreckage was discovered at a depth of 3900 meters [WHOI] which is at approximately $39 \mathrm{MPa}$ or 389 atmospheres of pressure. 


\subsubsection{Corrosion}

Corrosion due to seawater is an electrochemical process that is based on the electrical potential created when a particular material is introduced into the salinity of ocean environment. Because of the nature of the corrosion, different materials offer varying degrees of corrosion protection as displayed in figure 10 Not all corrosion resistant materials are suitable for the design constraints on a system however, and a well-studied tradeoff between corrosion resistance, workability, strength and weight must be considered. One common material for oceanographic use is Type 316 stainless steel. It is relatively inexpensive, easy to machine and weld, and readily available. It presents a moderate deterrence to crevice and pit corrosion, due to the presence of chromium and nickel in the alloy, and exhibits an incubation time in seawater of six months to one year ${ }^{3}$. On the other end of the spectrum is titanium, which is relatively expensive, difficult to manufacture, and requires great skill to machine and weld. The tradeoff is that it is extremely corrosion and pitting resistant and very strong. The $1.9 \mathrm{~cm}$ thick titanium pressure hull on Alvin, for example, is rated to withstand the pressures at a depth of 4500 meters (from http://www.whoi.edu/page.do?pid=9915).

\footnotetext{
${ }^{3}$ From http://www.corrosioninst.com/Materials Stainless Steel Intro.html
} 


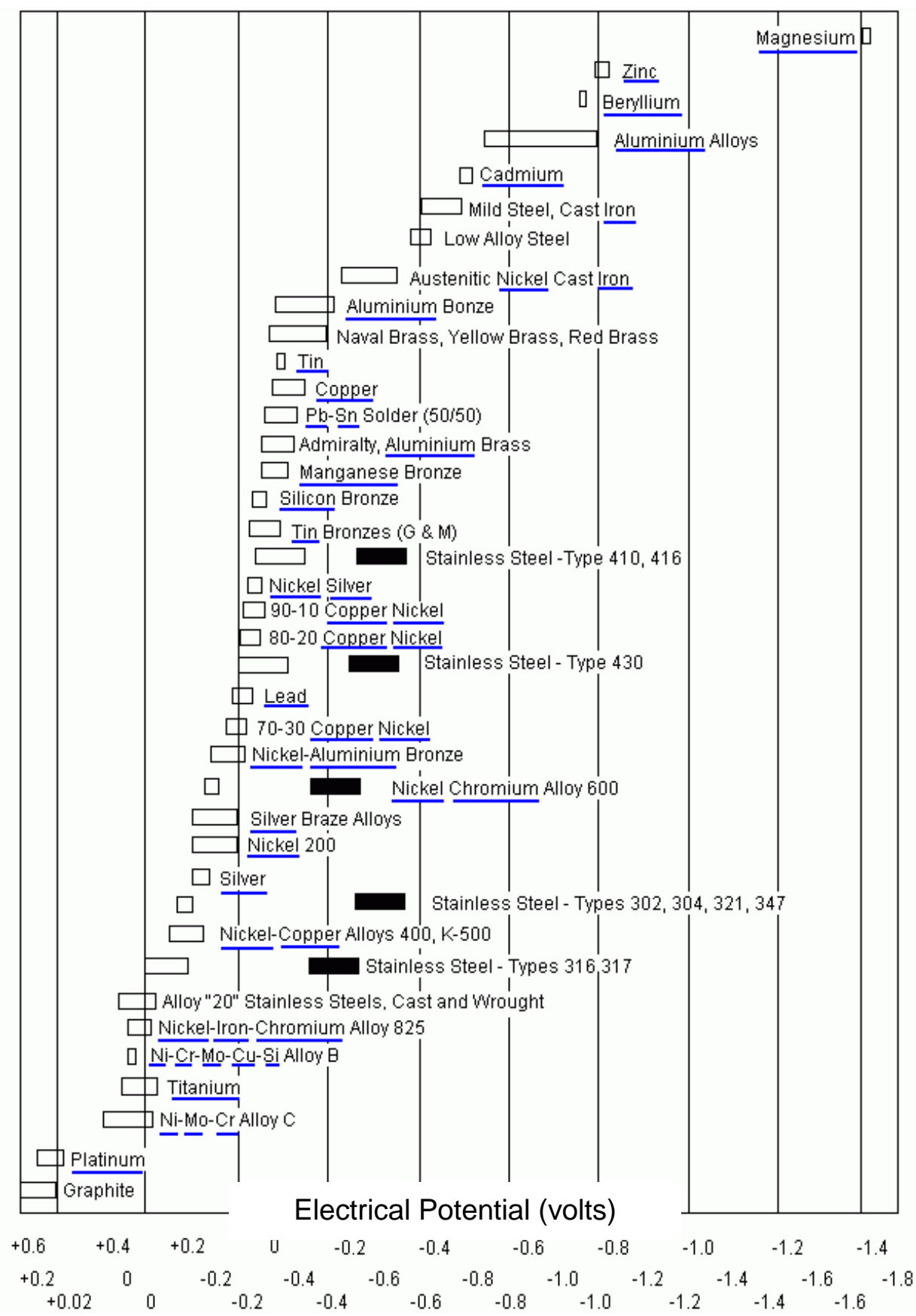

Figure 10 - Corrosion potential for various materials. Corrosion susceptibility increasing from left to right. The materials used most in underwater vehicle and system design (from least corrosion susceptibility to most) are titanium, stainless steel and aluminum (redrawn from: http://www.corrosionsource.com/handbook/galv series.htm). 
One way to use lower corrosion resistant and therefore cheaper and more readily available materials in oceanographic systems is by the use of sacrificial anodes. These anodes are normally made of magnesium which has a very high negative corrosion potential (see figure 10). This property ensures that the magnesium anode will be corroded by the seawater environment prior to any other nonmagnesium component, hence the term sacrificial.

\subsubsection{Power Requirements}

As the field of underwater vehicles moves more into the area of autonomous operation, power requirements versus availability is a priority calculation. I will not provide an in depth discussion on this topic, only to note that it must be taken into consideration during any underwater vehicle system design. Power considerations are the most dominant consideration during the design of small autonomous underwater vehicles, as it is the most limiting resource on these vehicles [Bradley, et al].

\subsection{A Physical Design Approach}

Since the introduction of the commercial camera flash in the 1930's [Nayar], photographers have attempted to tackle an ever increasing array of challenging environments. As oceanographers expanded the quest for greater depths outside the reach of ambient light, more complex methods of lighting were developed to provide adequate illumination. Unfortunately, the lighting technology has failed to keep pace with the existing need and flash blocks, 
strobes and flood-lighting continue to be the dominant methods of deep water lighting. These existing methods have limitations that include high power requirements, uneven intensity across the field of view and shadowing (especially from moving platforms or systems with large source / receiver separation). In imaging distances dominated by absorption artificial lighting also produces results with a blue-green hue.

My design approach for a robust and dynamic lighting system was to tackle the problem from a different perspective than those previously presented. How can one physically interact with the optical properties of the ocean (both inherent and apparent) while at the same time minimize the limitations of existing artificial lighting systems? The notional and immediate answer was to physically minimize the effects of seawater on the propagation of light by reducing the scatter interaction volume using dynamic lighting. 


\subsection{Scatter and Illumination Volumes}

As shown in section 2.1.1, source / receiver separation is a quick and effective way to minimize the scatter interaction volume in a participating medium. One can further decrease the interaction volume by the using dynamic or sweeping lighting techniques, as graphically shown in figure 12 .
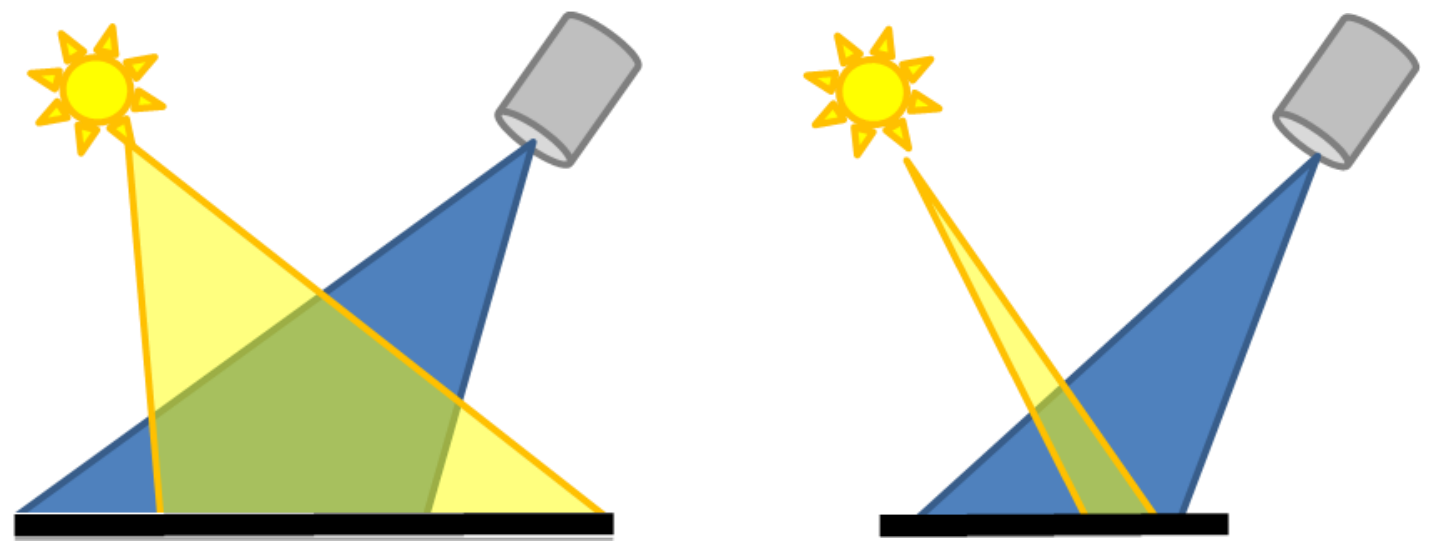

Figure 11 - Dynamic scatter interaction volume based on dynamic lighting. The scatter interaction volume is greatly decreased by limiting the field of illumination of the source. The interaction volume size remains constant (but reduced) as the smaller light beam is swept across the target to ensure a resulting final image that includes the majority of the intensity information available.

The scatter interaction volume is greatly decreased by limiting the field of illumination of the source. The interaction volume size remains constant (but reduced) as the smaller light beam is swept across the target to ensure a resulting final image that includes the majority of the intensity information available. 


\subsection{Scope}

As in the Jaffe experiments [Jaffe 2010], I chose to limit the scope of absorption affects in my research and experimentation and instead concentrated on backscatter vice contrast limited environments. Even though low contrast imaging environments still exist even with little or no backscatter present, these specific situations are beyond the scope of this thesis. Contrast limited imaging are currently studied by various researchers interested in the effects non-linear color attenuation and the associated enhancement and mitigation techniques. I chose to limit the absorption considerations in this thesis because the colocated and final optical systems used in conjunction with the proposed dynamic lighting system can be built to greatly minimize absorption through both camera design and specialized optics. In addition, by limiting the scope of absorption in my design, I was able to significantly limit the complexity and cost of my experimental setup, while still providing suitable data.

\subsection{Decreased Limitations}

My initial approach was constructed to remove some of the limitations that exist with current computer vision and other physical lighting methods. There have been many approaches to minimizing the lighting system effects in deep ocean systems with most concentrating on computer vision and filtering concepts [Garcia, et al]. The dynamic lighting system (DLS) concept can be easily manipulated to change lighting patterns and / or shape to uniformly distribute 
intensity throughout the target plane and the color can also be changed (realtime if necessary) to maximize imaging distance by minimizing absorption. In addition, this system does not need to know the distance to the target or the scattering properties of the scene and does not rely on robust assumptions, such as homogeneous media or single-scattering only. It also is on track with emerging modular design concepts [McFarlane] of underwater vehicles, in that it fulfills the requirement of a more elaborate illumination technique while limiting or eliminating source / receiver separation requirements

\subsection{Components}

To reiterate, the design concept for the dynamic illumination system was an easily adaptable add-on illumination system with a small physical foot print, low power consumption that was comparably inexpensive and consisted of open sourced and / or commercial-off-the-shelf (COTS) components.

After researching available COTS equipment, I initially chose the Beagle Board, a low-cost OMAP3530 based processing and control board and the Texas Instruments DLP pico projector development kit $^{4}$. The major design specifications of these components are available in Appendix $C$ and the original development block diagram for both the beagle board and the pico projector is shown in figure 13.

\footnotetext{
${ }^{4}$ All component specifications are provided in Appendix C
} 


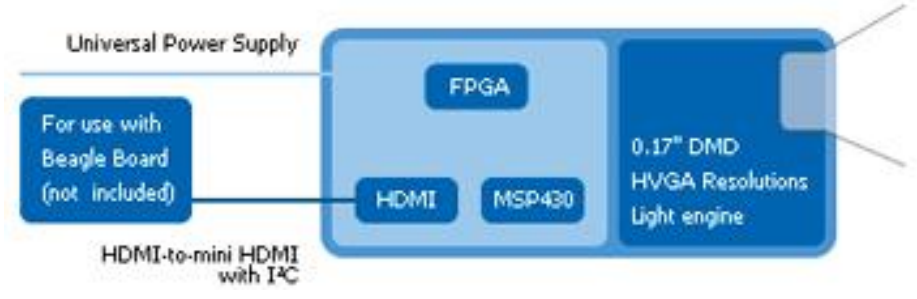

Figure 12 - Beagle Board / Pico projector original developer block diagram. The pico projector incorporated digital light projection (DLP) technology and was made specifically for use with the beagle board. Combined they met the majority of my criteria as the system was low-power, COTS, small, and relatively inexpensive

I chose this setup originally as the pico projector incorporated digital light projection (DLP) technology and was made specifically for use with the beagle board. Combined they met the majority of my criteria as the system was lowpower, COTS, small, and relatively inexpensive. The processing board and projector are shown in figure 14 in actual size.
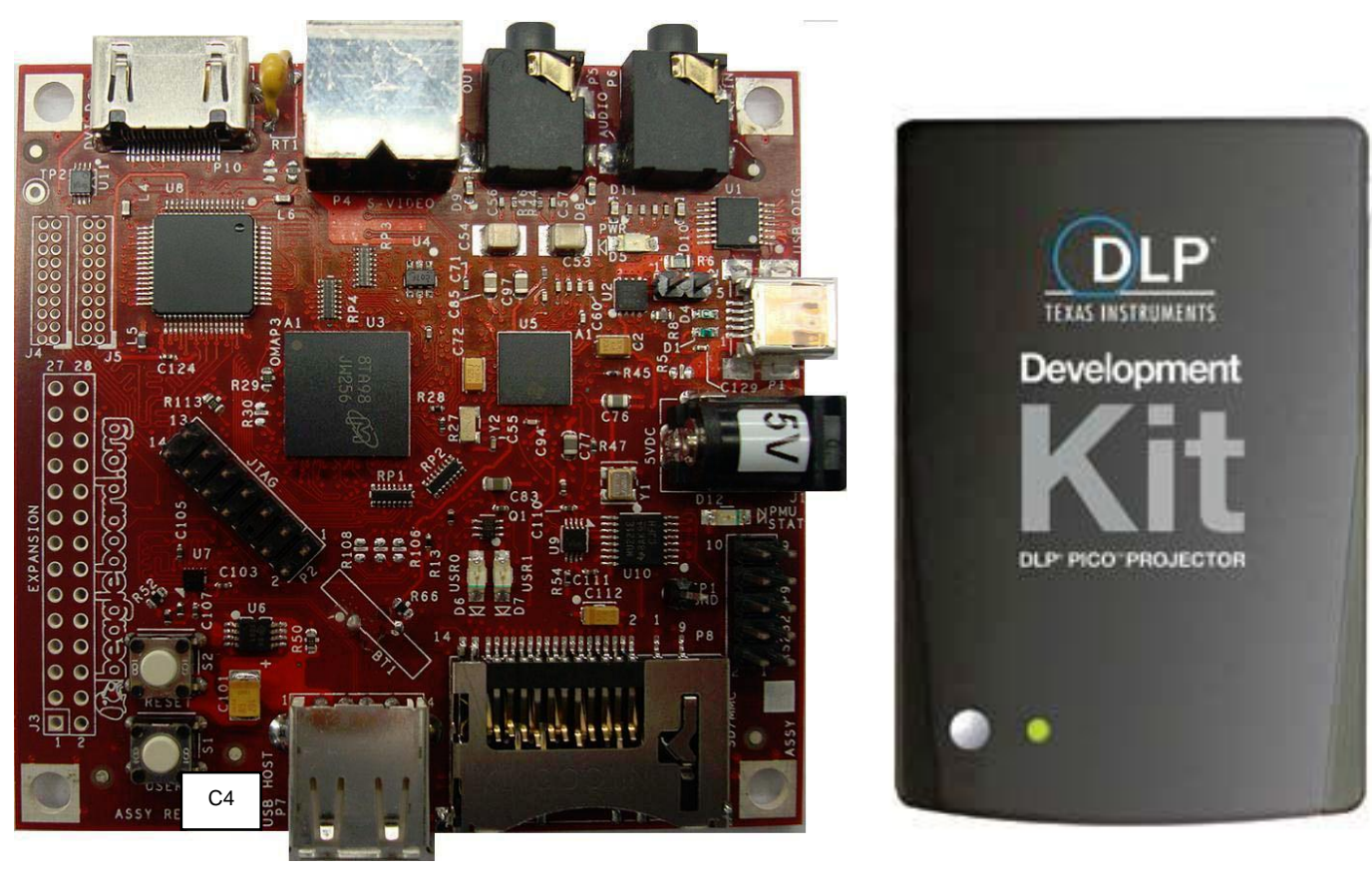

Figure 13 - BeagleBoard processor board and Texas Instruments DLP Pico projector shown in actual size. 
Although the pico projector and beagle board development combination appeared to satisfy the initial design criteria, it ultimately did not lend to the final system design. I was forced to add an expansion kit to the beagle board to provide the minimum amount of control interfaces necessary to prototype the illumination system. Specifically, the expansion board added a battery backup for the system clock (ultimately important for real time image catalogue), an additional SD card slot for storage, an onboard 10/100 Ethernet port and a RS232 serial port that I used extensively to troubleshoot and debug the system. The initial prototype component setup is shown in figure 15.

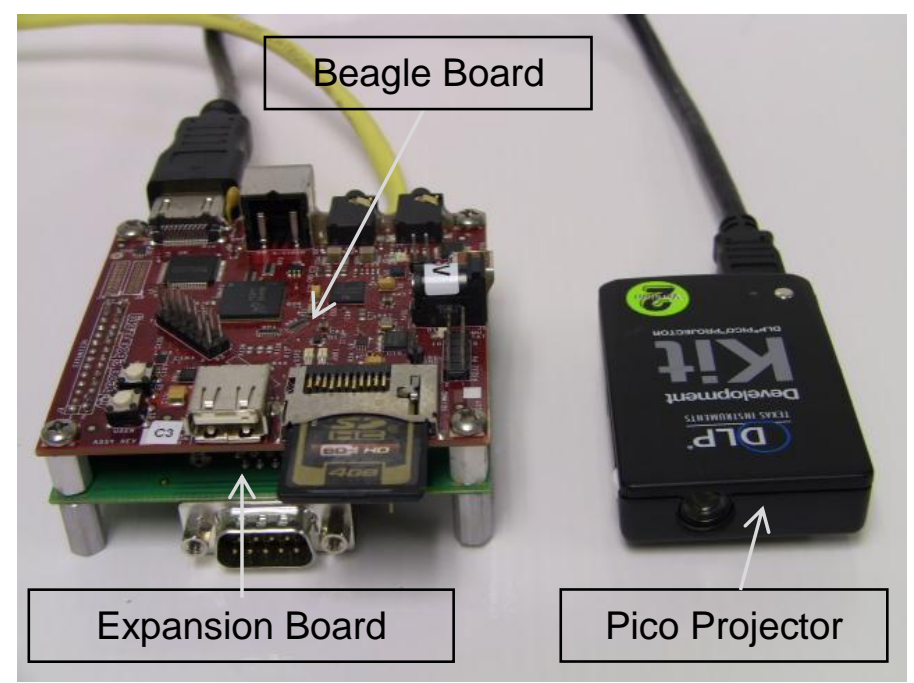

Figure 14 - Initial dynamic lighting system prototype. The expansion board was a required addition to the original system to provide the minimum amount of control interfaces necessary to prototype the lighting system. Specifically, the expansion board added a battery backup for the system clock (ultimately important for real time image catalogue), an additional SD card slot for storage, an onboard 10/100 Ethernet port and a RS232 serial port that I used extensively to troubleshoot and debug the system. 
In order to test the initial prototype, I determined how to make the components work together as a lighting system. In order to fulfill the low-cost and adaptability design constraints, an open sourced operating system was selected as a development platform. I installed and tested the prototype with both the Ubuntu and Angstrom distributions of the Linux operating system as well as the Android smartphone operating system. I ultimately picked Ubuntu based on the widely available support community and because it was the distribution that I was most familiar with.

Upon successful operation of the beagle board on Ubuntu I moved onto the task of software development. The software had to be easy to use and sweep a user selected shape of user selected size across the field of illumination. I wanted the code to be very simple initially, for debugging purposes, but still be able to provide the means to select the shape, size, speed, path and color of the illumination pattern. In the end I used the Simple DirectMedia Layer (SDL) library and $\mathrm{C}++$ to code the illumination steering and shape selection algorithm.

\subsection{Experimental Setup}

The next step in the design evolution was creating a proof of concept. In order to conduct this step, a simple experimental setup was constructed out of a 20 gallon glass aquarium. I chose a twenty gallon aquarium $(76.2 \times 30.5 \times 38.1 \mathrm{~cm})$ specifically based on the relatively short optical path length the lighting source would encounter. This small path minimized the absorption of the light and 
allowed me to concentrate on scatter effects only, as per the scope of this design. A general layout of the testing is shown as figure 16 and is the same setup that was used throughout the evolution of the lighting system laboratory experiments. To mimic a scattering environment I used Gold Bond body powder for decreased visibility with occlusions and Maalox for a generalized turbid water environment mixed with fresh water. I measured the turbidity for reference only using a Secchi disk turbidity tube.

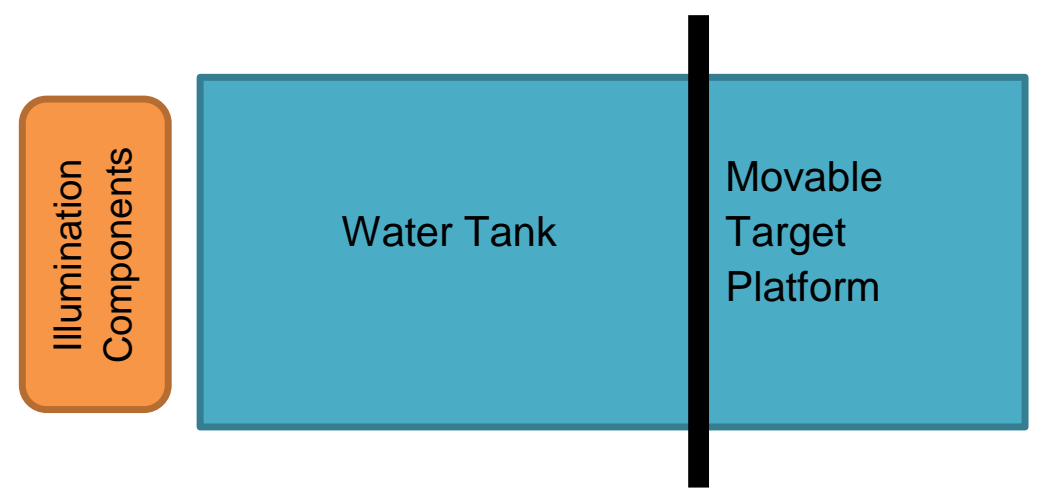

Figure $15-$ General testing setup. The testing setup consisted of a twenty gallon aquarium $(76.2 \times 30.5 \times 38.1 \mathrm{~cm})$ chosen specifically based on the relatively short optical path length the lighting source would encounter. This small path minimized the absorption of the light and allowed me to concentrate on scatter effects only, as per the scope of this design. The target platform was movable and could provide an illumination path length from less than $1 \mathrm{~cm}$ to just over $76 \mathrm{~cm}$. 


\section{CHAPTER 4: DATA COLLECTION AND ANALYSIS}

\subsection{Initial Proof of Concept}

To test the lighting system I compared an image of a resolution chart used for ISO 12233 photography, illuminated by flood lighting (un-collimated white light from the pico projector) in turbid water to the image constructed from dynamic lighting of the same scene in the same environment. All images in the research experimentation were taken by a Canon Vixia HFS10 in cinematic mode (60 fps), with automatic white balance and focus, at 1440 by 1080 resolution and a source / receiver separation of less than $10 \mathrm{~cm}$. Although a larger source and receiver distance would improve scatter minimization and ultimately system performance, I used a minimal SRS to concentrate on the dynamic lighting aspect of contrast improvement and to reduce the system footprint. The target was placed at the maximum experimental distance of $76.2 \mathrm{~cm}$. The ground truth test was the comparison of a flood lit image (first frame of the video prior to dynamic lighting start) and one frame of a dynamically illuminated scene as shown in figure 17. The flood lit illumination was provided by the pico projector at full power $(\sim 7$ lumens) and the dynamic lighting pattern was a 100 by 100 pixel white square. The turbid water environment consisted of fresh water mixed with gold bond powder, equating to approximately $5 \mathrm{NTU}$, as determined by a clear turbidity tube. Although hard to see via printed image, there is a minimization of scatter 
effects, visualized as an increase in contrast, in the dynamically illuminated image on the right.

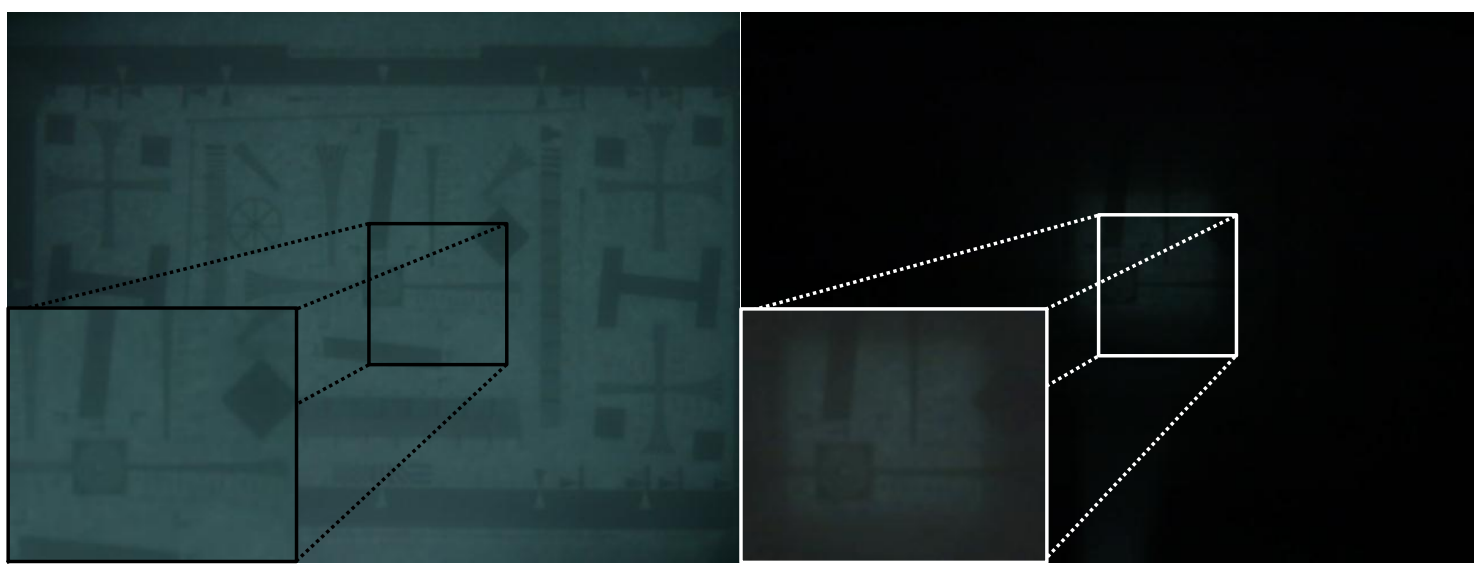

Figure 16 - Initial ground truth testing with flood lit scene on the left and dynamically illuminated scene (on the right) in a scattering environment. ${ }^{5}$ The dynamically illuminated scene on the right shows some subjective increase in contrast when compared to the original image.

The full proof of concept experiment was conducted in a manner similar to the ground truth testing with results shown in figure 18. A turbid water environment was simulated using gold bond powder, this time equating to approximately 5.5 NTU (as per turbidity tube measurement) and compared the flood lit and the dynamically illuminated target images.

To create the final dynamically illuminated image, I utilized a MATLAB code that compared the pixels of subsequent frames of the acquired target video (dynamically illuminated) and built a mosaic final image based on the brightest of

\footnotetext{
${ }^{5}$ Dynamically illuminated image corrected for review by print only, un-corrected images used only for quantitative comparison.
} 
each compared pixel; it is similar to the superimposition method proposed by Jaffe [Jaffe 2010]. The result, in theory and based on the ground truth results, would be an image of the original scene with an increased contrast and reduced scatter effects.

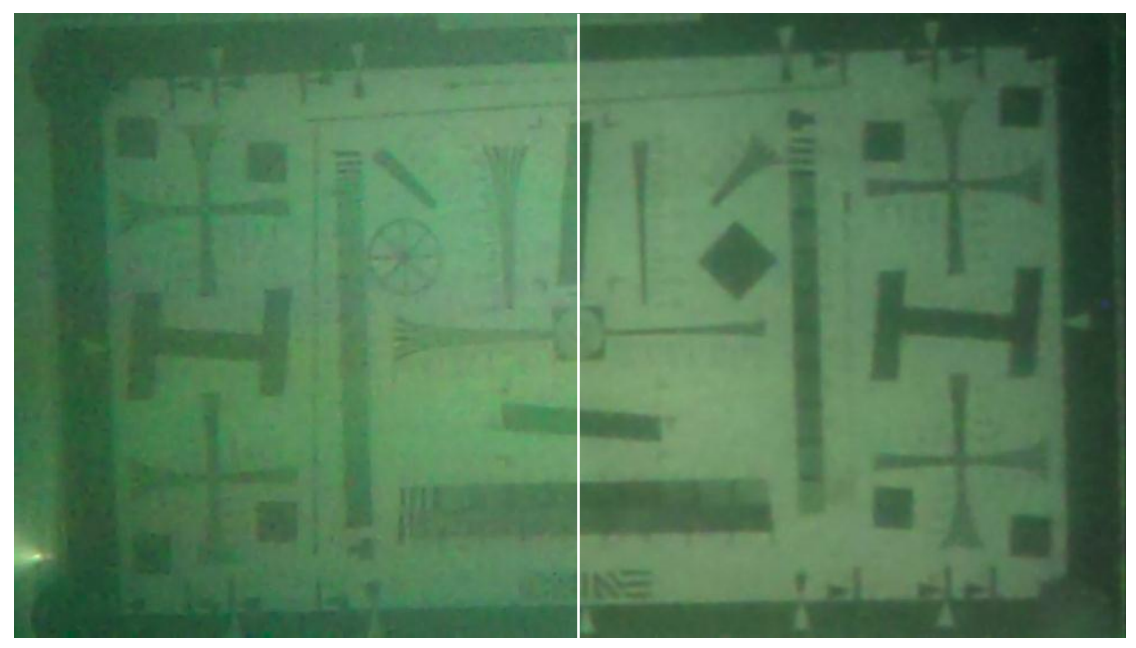

Figure 17 - Initial proof of concept utilizing Beagle Board Rev C4 and Texas Instruments DLP Pico Projector at maximum power. Original flood lit scene on the left and reconstructed dynamically illuminated scene on the right. Notice the green hue of each image due to absorption in water tank. . Although a good proof of concept, the illumination intensity was not sufficient enough for the ultimate dynamic lighting system.

The proof concept of the initial prototype was both exciting and disappointing. The system worked exactly as designed but the results were less than expected. A subjective quality assessment of the two images would allow that the reconstructed image (figure 18 refers) showed a slightly better contrast than the original. A quantitative assessment utilizing the mean gradient magnitude, showed the dynamically illuminated image to be overall exhibiting increased contrast. The mean gradient magnitude comparison is shown in figure 19. The 
pixelation artifacts in both the original and reconstructed images was an indication of the low intensity of illumination provided by the pico projector as both a flood lit source and even when used as a dynamic illuminator. These results clearly showed the need for a brighter illumination source that still met the self-imposed constraints of the design criteria.

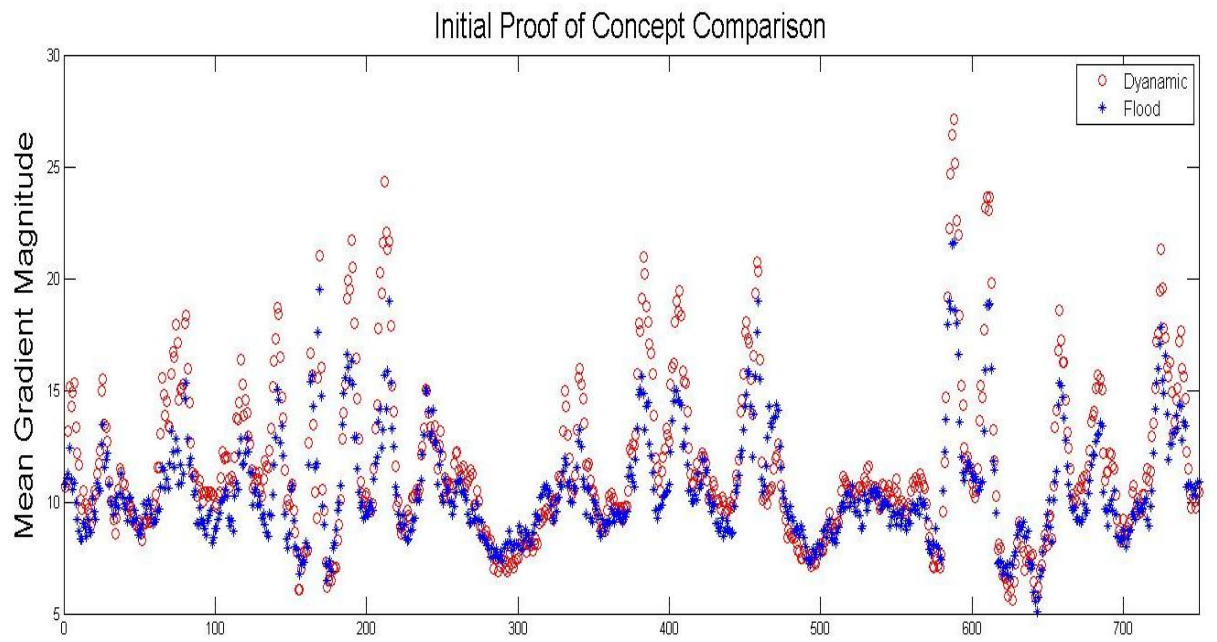

Figure 18 - Initial proof of concept mean gradient magnitude comparison. The comparison was conducted with the outer pixels removed to prevent false readings from water tank reflections and edges of scene target.

\subsection{Revised Proof of Concept}

Over the two years of research and design of the dynamic lighting system were great leaps in DLP technology and increased availability of high-quality, inexpensive and powerful hand-held projectors. The limitations of the original TI pico projector were significant enough to not use it as a final component in a deployable dynamic lighting system. I researched available commercial products and settled on the Optoma PK201 and PK301 as viable options to continue with 
design and specifications. The optoma projectors were powerful projectors (20 and 50 lumens respectively) that still maintained the advantages of small size, operated on LED DLP technology and had low-power consumption. In addition, the new projectors included lithium ion rechargeable batteries for increased flexibility in future power system design.

Upon receipt of the new projectors I re-ran the ground truth and proof of concept experiments. Each experiment was conducted under the same conditions as the originals with results using the PK301 projector at 50 lumens provided in figures 20 and 21.

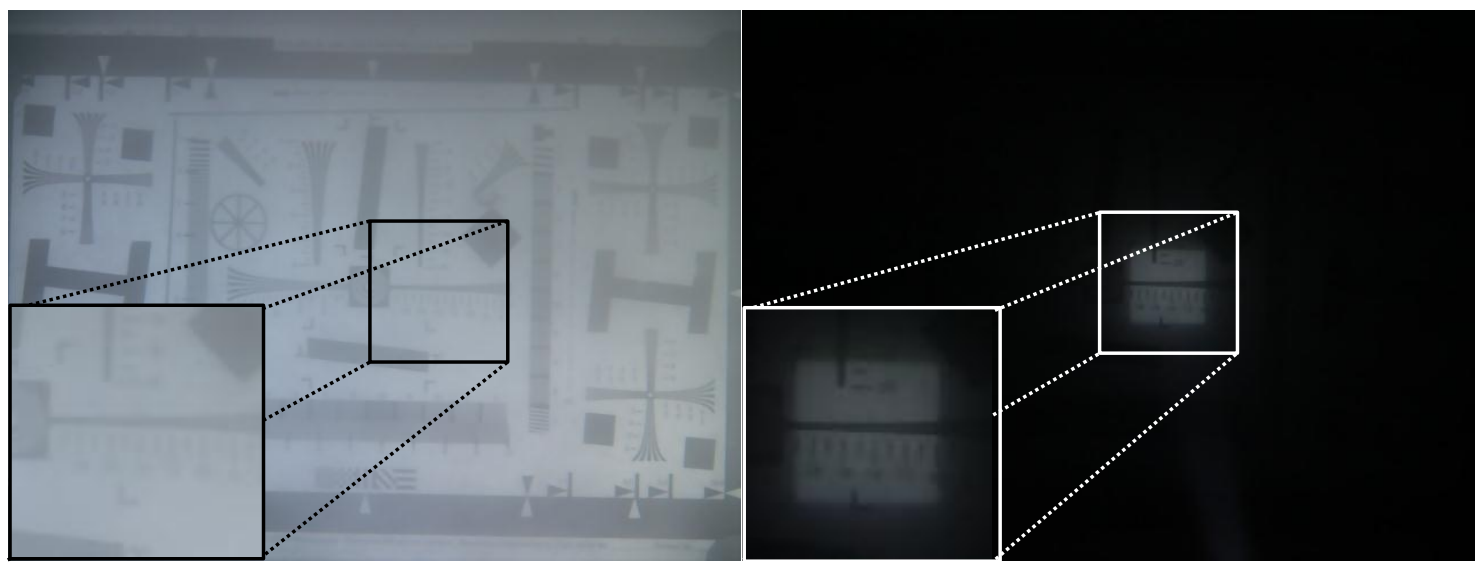

Figure 19 - Revised ground truth testing with flood lit scene (on the left) versus dynamically illuminated scene (on the right) in a scattering environment. An increased contrast is already readily apparent in the dynamically illuminated scene with a marked decrease in the lighting halo around the illumination pattern exhibited in the pico projector. 

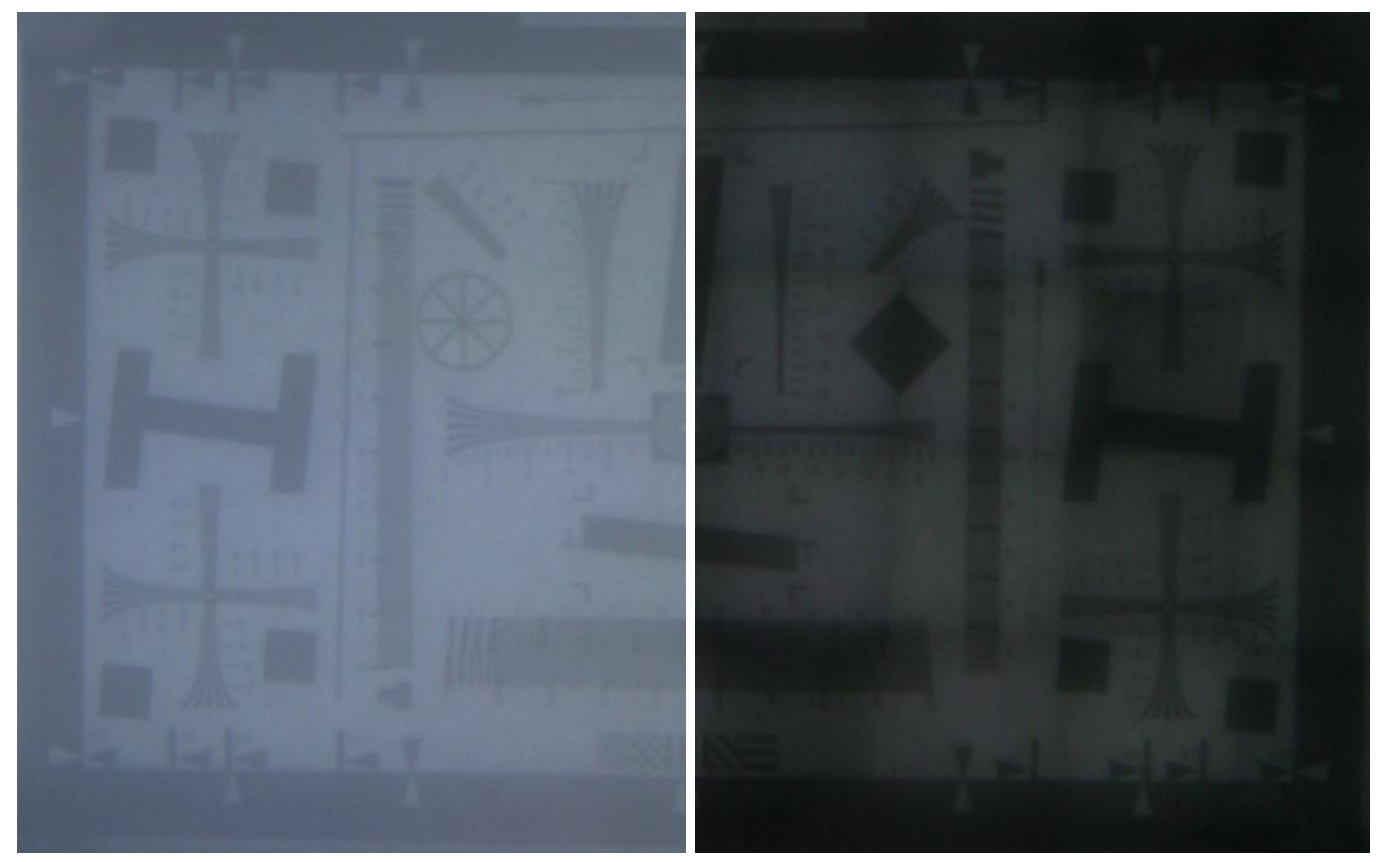

Figure 20 - Revised proof of concept with new lighting source (PK301) at full power of 50 lumens. There is a marked increase in contrast and lack of the "milky veil" caused by scatter. The square artifacts in the reconstructed image are a reconstruction algorithm issue that was corrected for future experiments.

The new ground truth and proof of concept results were very encouraging, both experiments showed clear subjective improvement of a dynamically lit scene over a flood lit one. The higher contrast ratio of the new lighting source proved very important to prevent the halo around the illumination patterns experienced with the pico projector. The illumination pattern in the right side of figure 20 shows a crisp and clean distinction between source pixels that are on and those that are off. I was also able to show quantitative improvement in that the mean gradient magnitude of the dynamically lit scene was greater than the original scene (see figure 22). The dark banding artifacts on the dynamic lighting image (right side of figure 21) are a result of poor frame extraction from the original 
video and can be easily remedied by changing the extraction frame rate or the pattern sweep rate for future testing.

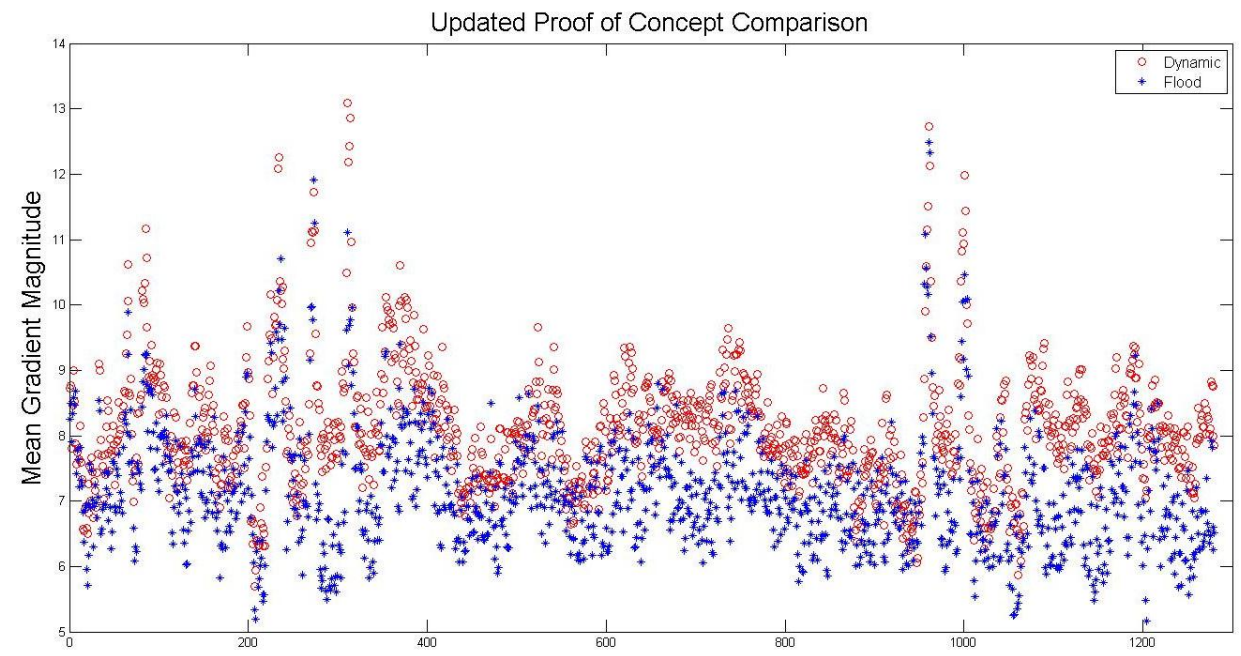

Figure 21 - Revised proof of concept mean gradient magnitude comparison. The comparison was conducted with the outer pixels removed to prevent false readings from water tank reflections and edges of scene target. The mean gradient magnitude improved over the entire image. 


\subsection{Bench Top Testing}

With the completion of a successful proof of concept, it was time to begin a battery of bench top laboratory tests that accumulated enough data for design analysis. In order to accomplish this, a bench top lighting system was constructed (as shown in figure 23) and used to dynamically illuminate the same resolution chart used in the proof of concept testing with various pattern shapes in various turbid water environments. The proof of concept pattern size and shape, a $100 \times 100$ pixel square, was based on the Levoy and Singh experiments in which a 90 pixel white square was utilized. Although this pattern worked adequately for the confocal imaging experiments, the boundaries of the current dynamic lighting system were unknown and still required formal definition.
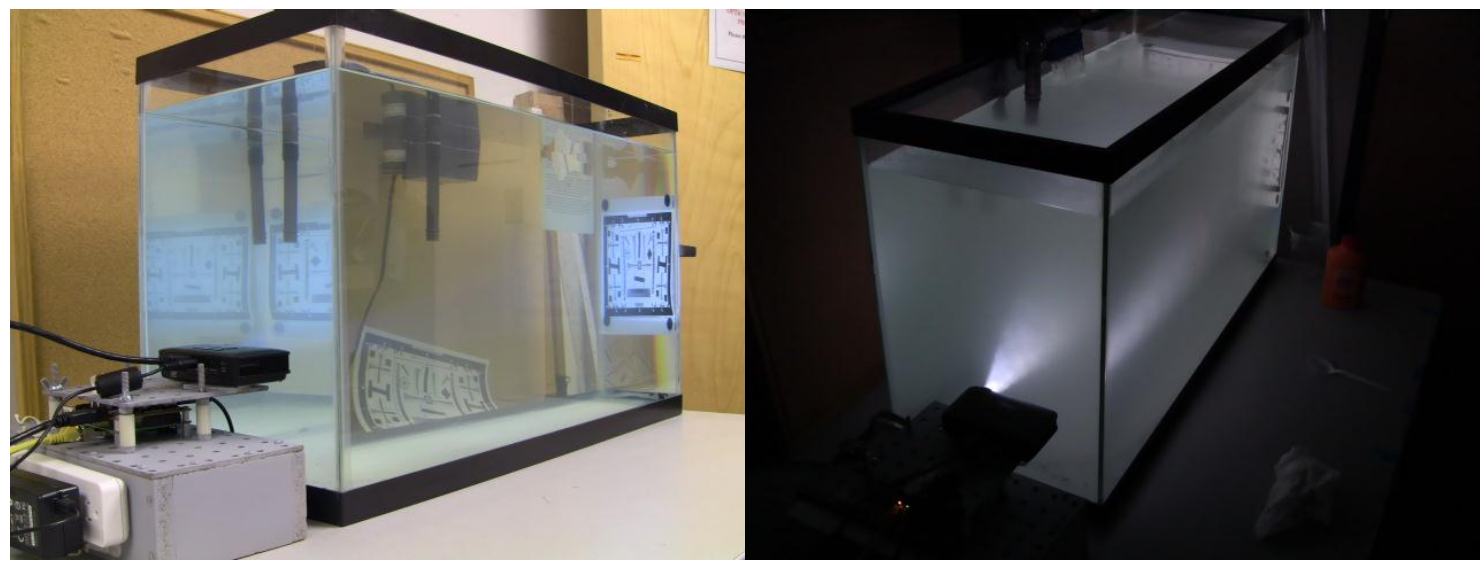

Figure 22 - Bench top testing experimental setup, with overall system shown on the left and the system in operation in a turbid water environment on the right. Notice that scatter interaction with the un-collimated projector illumination brightened the entire water tank and the area surrounding it. 
The bench top system testing was conducted in two parts. The first parameter to test was pattern size, followed by the turbidity limitations in which the system would operate effectively. The initial testing and that covered in this thesis, concentrated on various sized square patterns and vertical and horizontal oriented rectangles. The approach to this portion of the testing was an attempt to glean a point where there existed an appropriate trade-off between scatter minimization (contrast improvement), illumination intensity (pattern area) and image sweep time. The smaller patterns maximized the reduction in scatter effects, but also decreased illumination intensity and increased sweep times. The larger patterns provided the maximum illumination intensity and shortest sweep times, but did so with a larger scatter interaction volume and therefore decreased contrast improvement. As a point of reference, the 75 by 75 pixel square sweep time for the resolution chart at full distance was approximately 63 seconds, as compared to approximately 12 seconds for the 250 by 250 pixel square.

In addition to the different shaped patterns I also attempted to change the pattern color to gauge any change in the system success. I chose a green color that mimicked the green hue provided in the initial proof of concept images that exhibited absorption due to power limitations. I chose an area of the final image that consisted of white background only and determined that the average value in the green channel was 164 . I used this value to create a green pattern with the corresponding RGB values of $[0,164,0]$. 
The results are provided below in a specific layout as shown in figure 24. The first part of the results is provided for subjective analysis and consists of an image of the original flood lit scene, an image of the dynamically lit scene and a contrast adjusted image for printing purposes only, as some of the final images were difficult to see when printed on paper. The second part is provided for quantitative analysis, and consists of a comparison of the mean gradient magnitude of the entire original and reconstructed images, a mean gradient comparison of the center portion of each of the images and an additional graph showing overall gradient magnitude change. The gradient comparison of the image center is used to show gradient magnitude comparison in more fidelity as well as convey the amount of scene information reproduced in the final reconstruction. In each comparison graph throughout the testing dynamic lighting data is shown in red and flood lit data is shown in blue. As an additional layer of objectivity and as a reference, the results are also provided for a flood lit scene and dynamically illuminated scene in a minimally turbid water environment. The reference environment was fresh water directly from the tap, filtered by an aquarium charcoal and foam filter system for 24 hours. The turbidity of the resulting reference environment was less than 2.3 NTU as measured by a $140 \mathrm{~cm}$ clear turbidity tube and the scene was placed at maximum distance $(76.2 \mathrm{~cm})$. The zoomed quantitative comparison area is shown in figure 25 at actual size. 


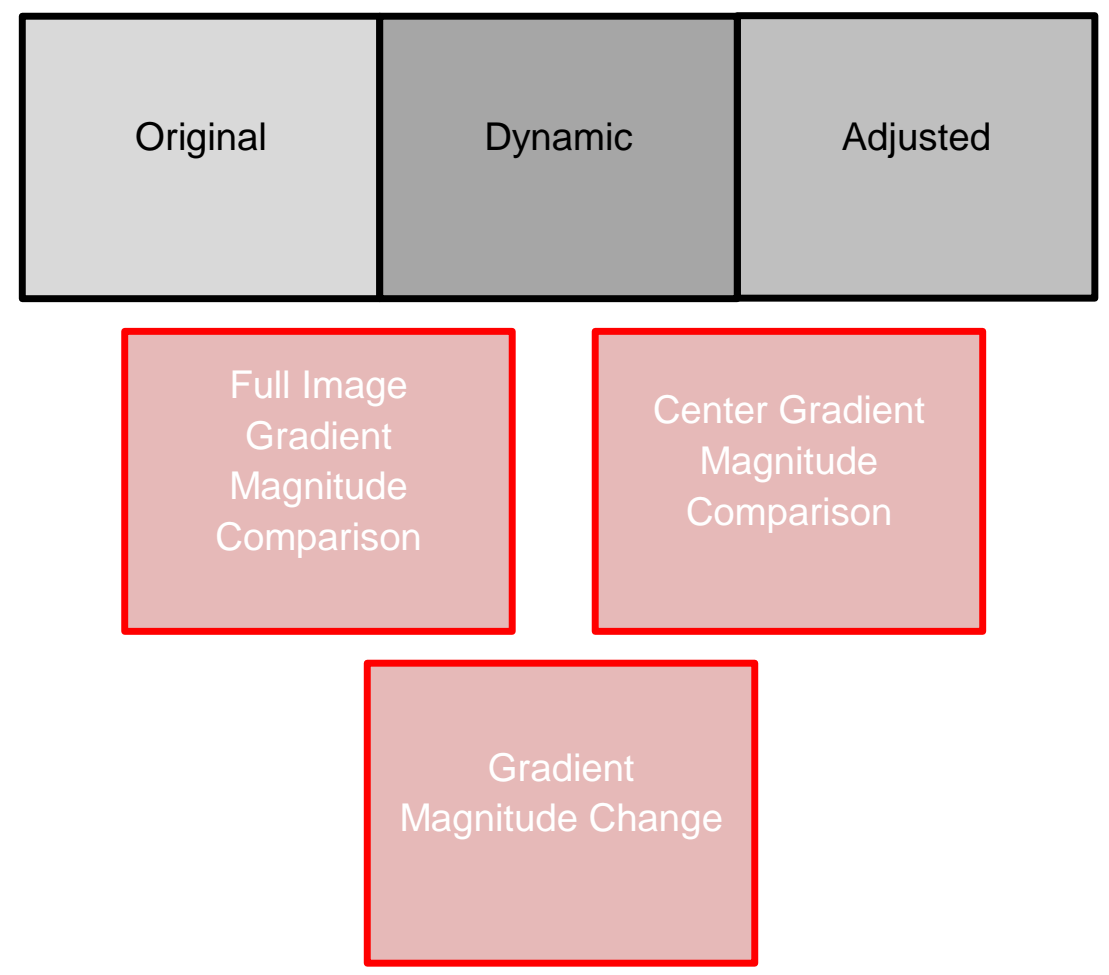

Figure 23 - Image and quantitative results layout. The first part of the results is provided for subjective analysis and consists of an image of the original flood lit scene, an image of the dynamically lit scene and a contrast adjusted image for printing purposes only, as some of the final images were difficult to see when printed on paper. The second part is provided for quantitative analysis, and consists of a comparison of the mean gradient magnitude of the original and reconstructed images, a mean gradient comparison of the center portion of each of the images and an additional graph showing overall gradient magnitude change. The gradient comparison of the image center is used to show gradient magnitude comparison in more fidelity as well as convey the amount of scene information reproduced in the final reconstruction. In each comparison graph throughout the testing dynamic lighting data is shown in red and flood lit data is shown in blue.

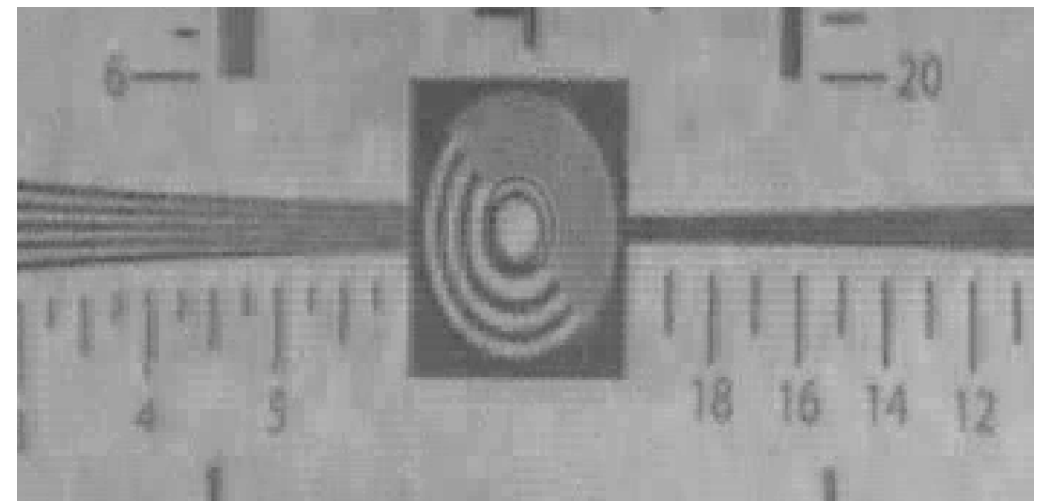

Figure 24 - Center gradient magnitude comparison image. 


\subsubsection{Images and Quantitative Results}

\section{Reference}
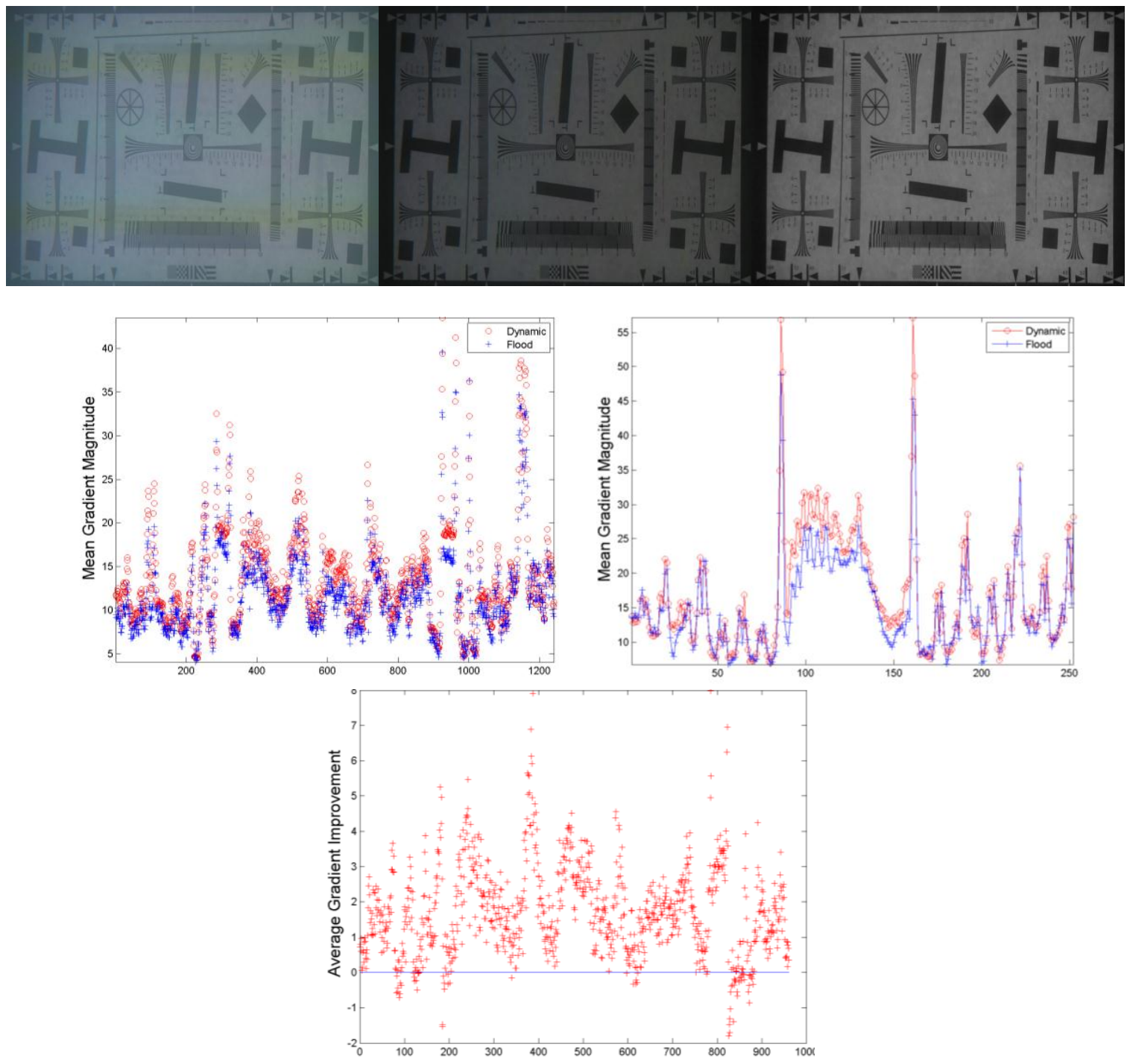

$100 \times 100$ pixel white square illumination at $<2$ NTU water - The reference test with white illumination showed an increase in overall contrast and provided a frame of reference for the imaging information included in the center portion of the scene. The mean gradient increase was 1.74. 
Green illumination
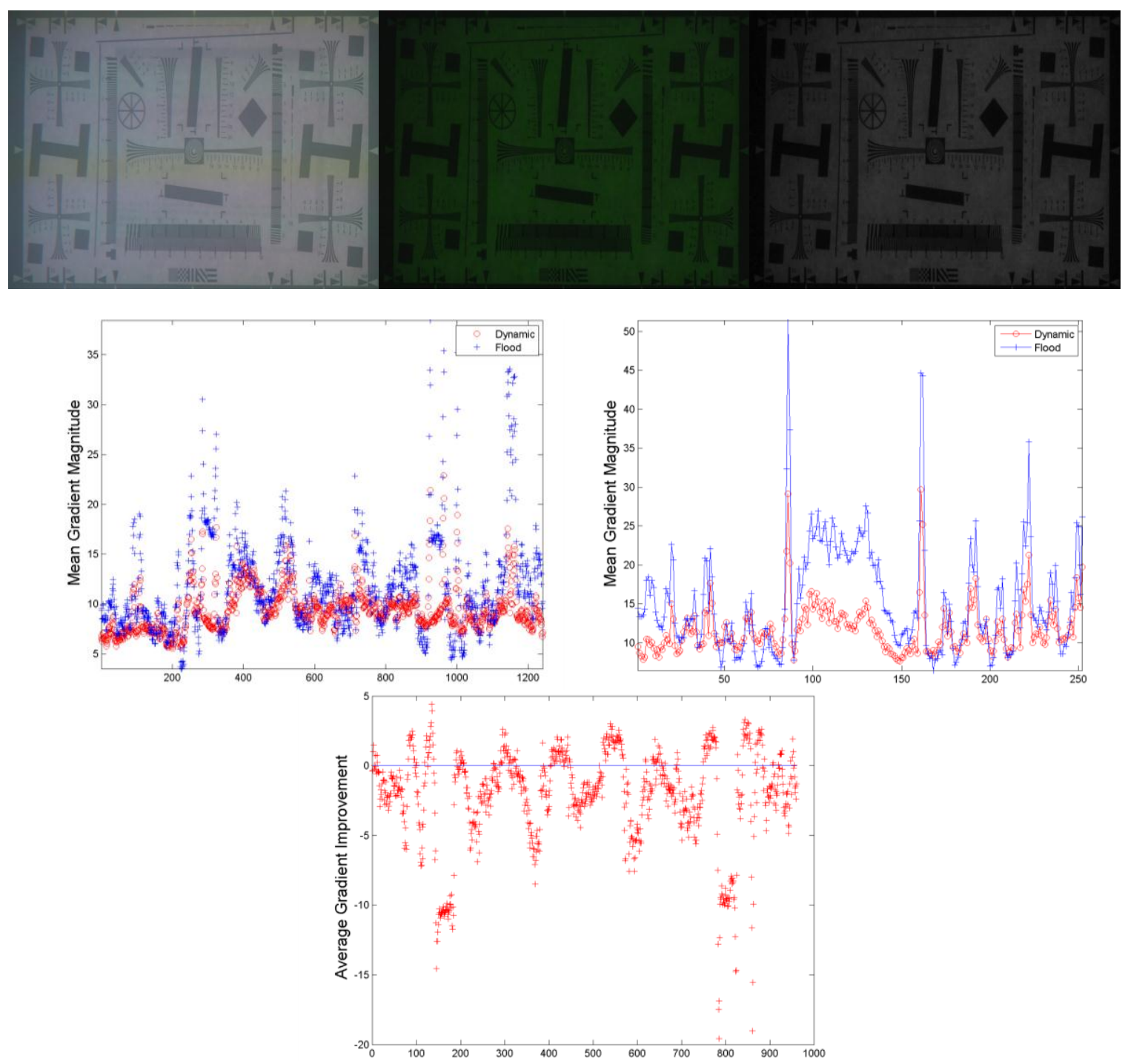

$100 \times 100$ pixel green square illumination at $<2$ NTU water - The reference test with green illumination showed no contrast improvement, but an adequate transfer of image information. The mean gradient decrease in the final image was over 2. The green illumination provided too low of an intensity illumination source for the imaging environment and contrast suffered for it. 
The reference testing and analysis provided future testing with some very clear and interesting guidance. The white light reference testing showed clear improvement between the original and dynamic scenes even with no visually noticeable turbidity. This was another great example of the utility of a dynamic lighting system, even in normal environments, the image quality is improved. On the other hand, the use of green colored illumination showed no improvement. The green illumination subjectively and objectively reproduced the original scene visual information, but did not improve the contrast in any portion of the final image. In a small experimental volume as provided by the water tank, absorption was treated as minimal and using illumination with a wavelength suspected to reduce absorption did not further any of the testing or design. It is clear the change in color merely reduced the illumination intensity and system effectiveness. Although the green illumination was attempted for all patterns and turbidity used in the bench top testing, the results will not be provided in the remainder of this thesis. The remainder of the results is shown below with all testing at maximum scene distance $(76.2 \mathrm{~cm})$ and turbidity of 5 NTU unless otherwise noted. 


\section{$\underline{\text { Square Patterns }}$}
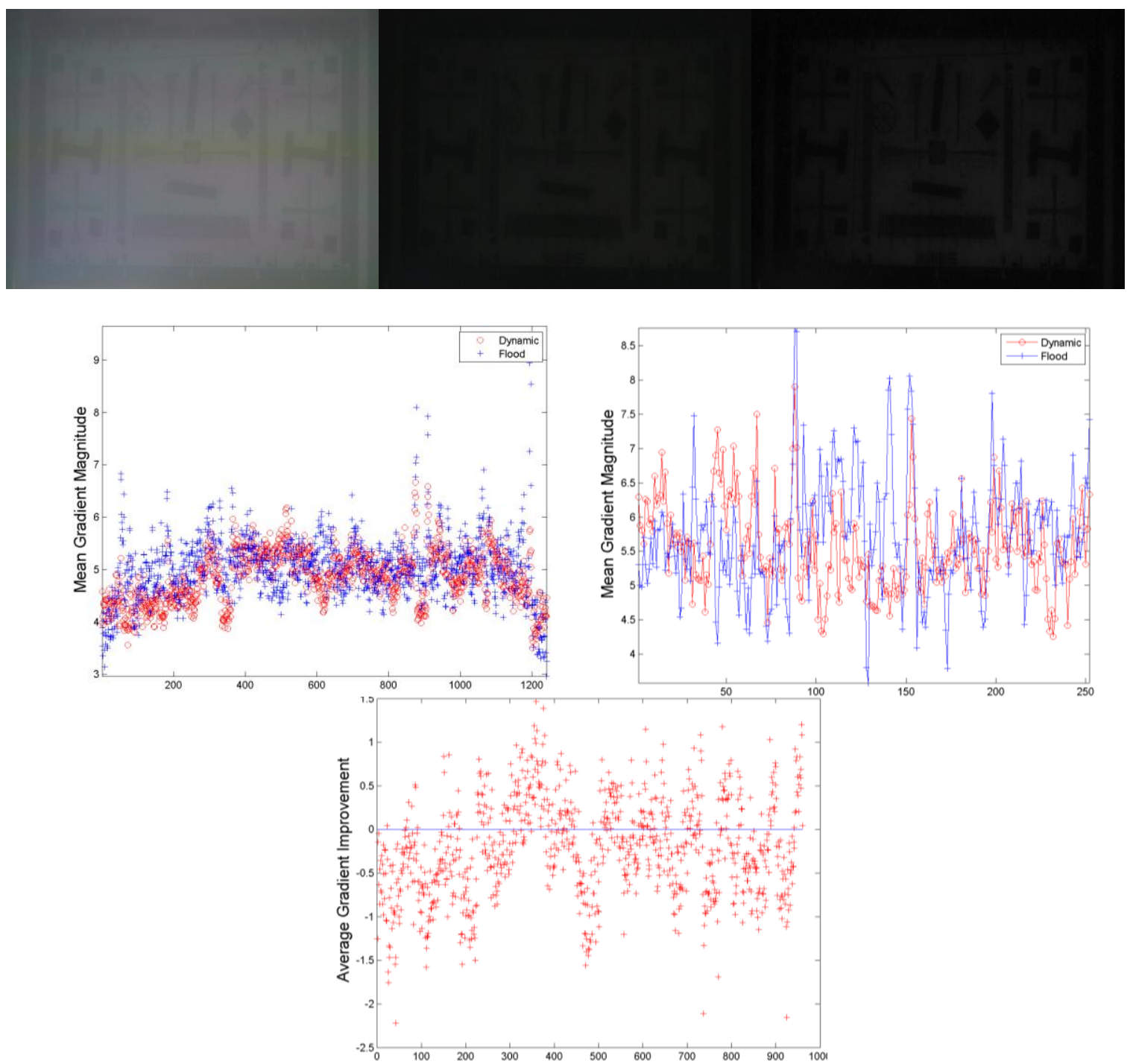

$75 \times 75$ pixel white square illumination at $\mathbf{5}$ NTU - The smallest pattern size showed some indication of contrast improvement, but original scene information was not reproduced due decreased illumination intensity provided by such a small illumination pattern. The mean gradient change was -.022 , or an overall decrease. 

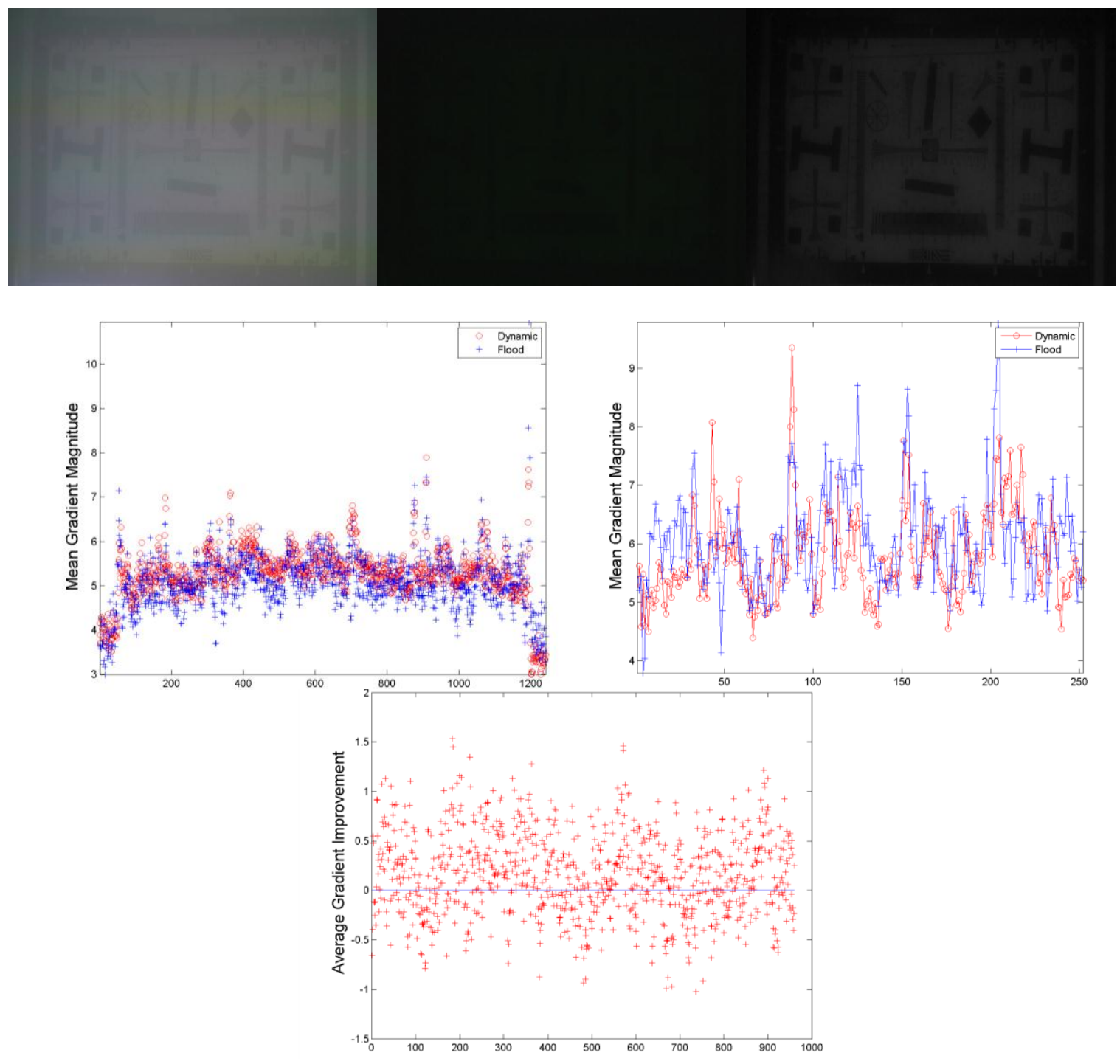

$100 \times 100$ pixel white square illumination at 5 NTU - The next pattern size showed increased subjective improvement that coincided with the objective results. The overall gradient magnitude showed improvement with an increased reproduction of the scene in the final image. Average gradient change was 0.16 or slight improvement. The current trend showed that as the illumination size increased (increased illumination intensity) the system showed greater effectiveness. 

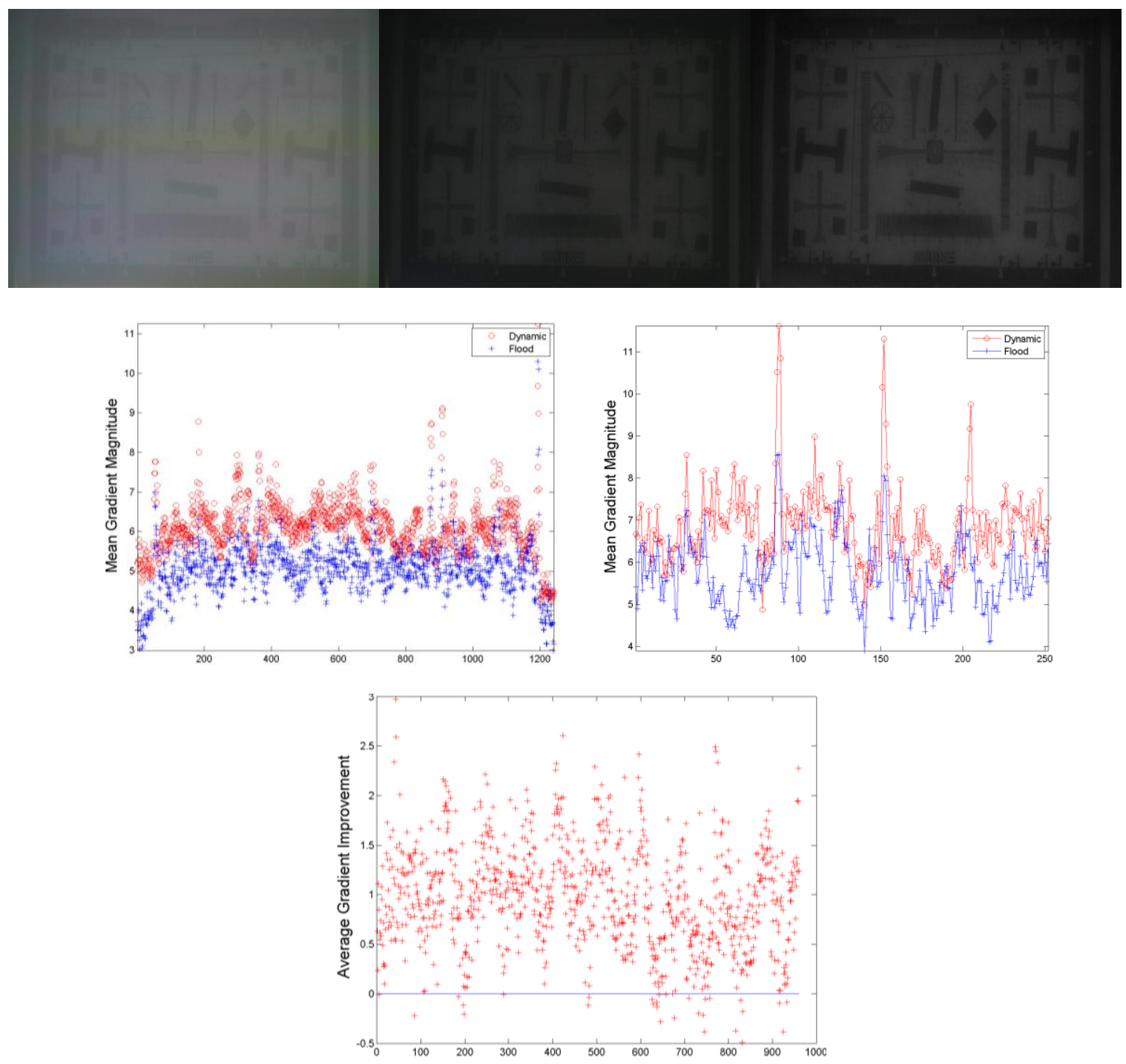

$150 \times 150$ pixel white square illumination at 5 NTU - This pattern showed a marked subjective and objective improvement in image quality. The final image appeared visually improved with adequate focus, increased contrast and great scene reproduction. The objective measure was on par with the appearance of the final image with marked gradient magnitude increase and superb scene information reproduction, especially when compared to the reference image. The gradient change was 0.97 or almost double the gradient magnitude of the original image. 
$250 \times 250$ pixels
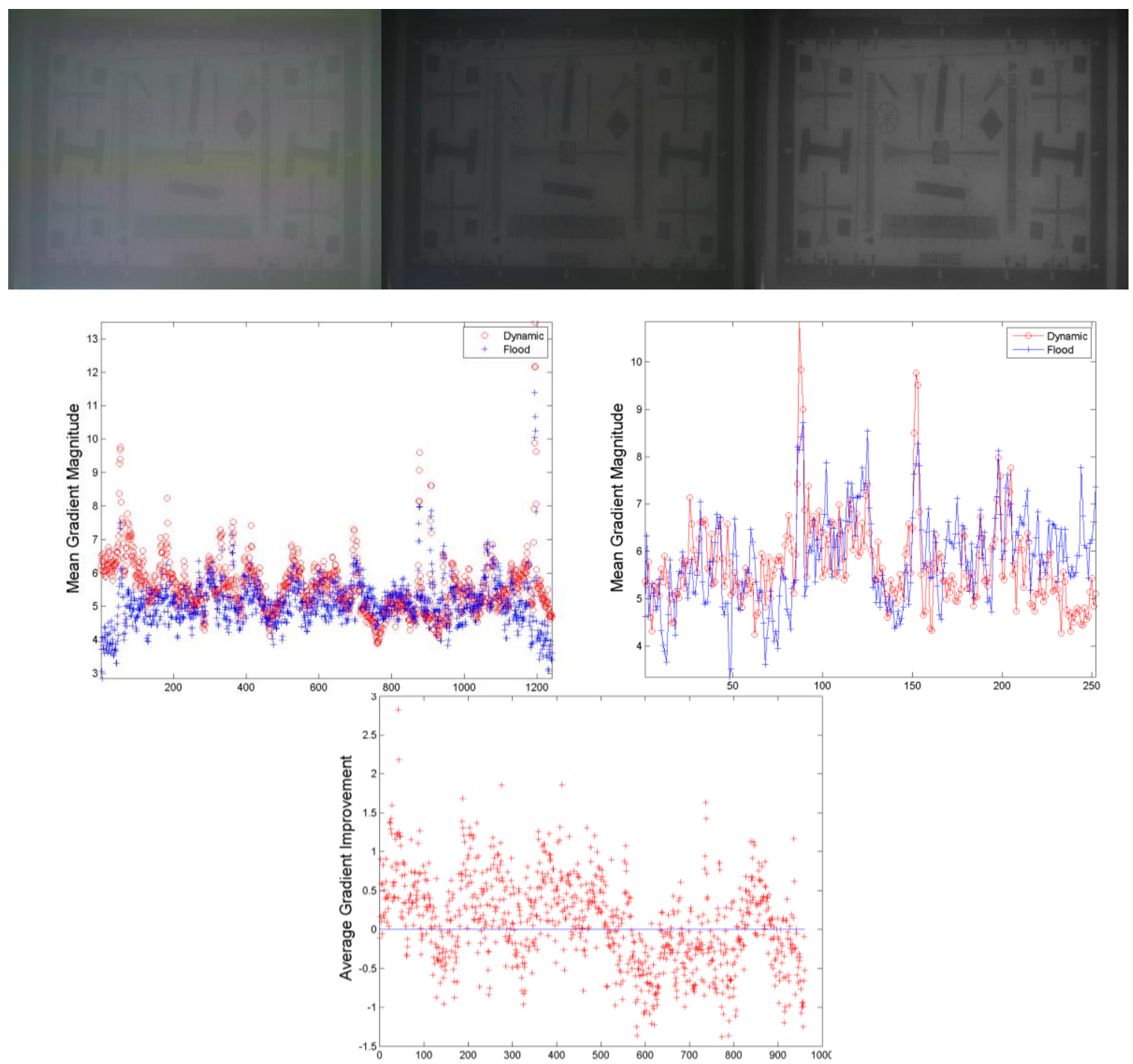

$250 \times 250$ pixel white square illumination at 5 NTU - This pattern marked a point that exceeded the tradeoff parameters measured in this testing. Although a subjective improvement, the gradient magnitude was not improved throughout the entire image and the original scene information was not as faithfully reproduced as in the previous pattern. The gradient change was 0.08 , which was less than the 100 pixel pattern, but showed greater scene information. 


\section{$\underline{\text { Horizontal Rectangle }}$}

The horizontal rectangle is 100 pixels high by 1280 pixels long and spans the entire width of the illumination volume while sweeping in the vertical plane as shown in figure 26. A vertical rectangle was not included in the initial testing, but is a method that will be approached with future testing.

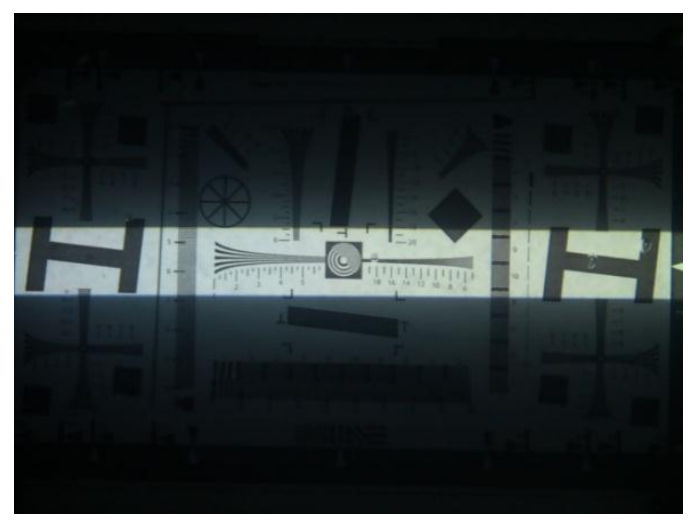

Figure 25 - Horizontal rectangle pattern. The horizontal rectangle is 100 pixels high by 1280 pixels long and spans the entire width of the illumination volume while sweeping in the vertical plane 

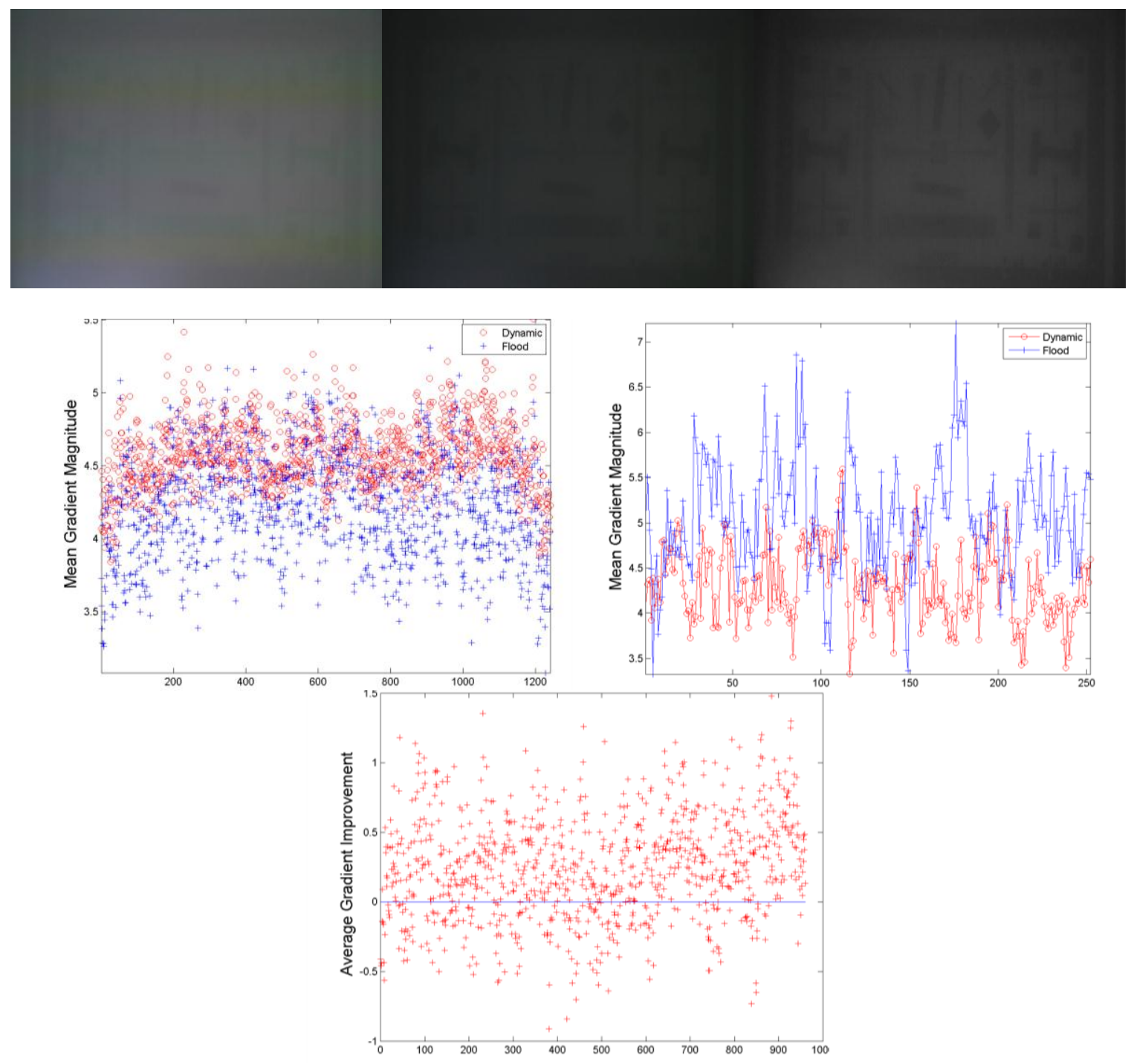

$100 \times 1280$ Horizontal rectangle at 7 NTU - The vertical rectangle pattern exhibited overall improved contrast. The subjective contrast improved, while objective results showed mild improvement consistent with the images. Scene information was reproduced more effectively that with the vertical rectangle with a gradient change of 0.24 , or a slight increase in contrast that was slightly greater than the $100 \times 100$ pixel square. 


\section{$\underline{\text { Turbidity Limits }}$}

The last portion of the initial design testing was an attempt to determine the turbidity limits of the dynamic lighting system. Several experiments were conducted with various degrees of turbidity to compare the limits to the effectiveness of the system. In the following tests a 100 by 100 pixel white square was used for dynamic lighting of the same resolution chart at $0,5,7,9$ and 15 NTU. The results are provided below with the reference and 5 NTU images included for ease of reading and comparison. 

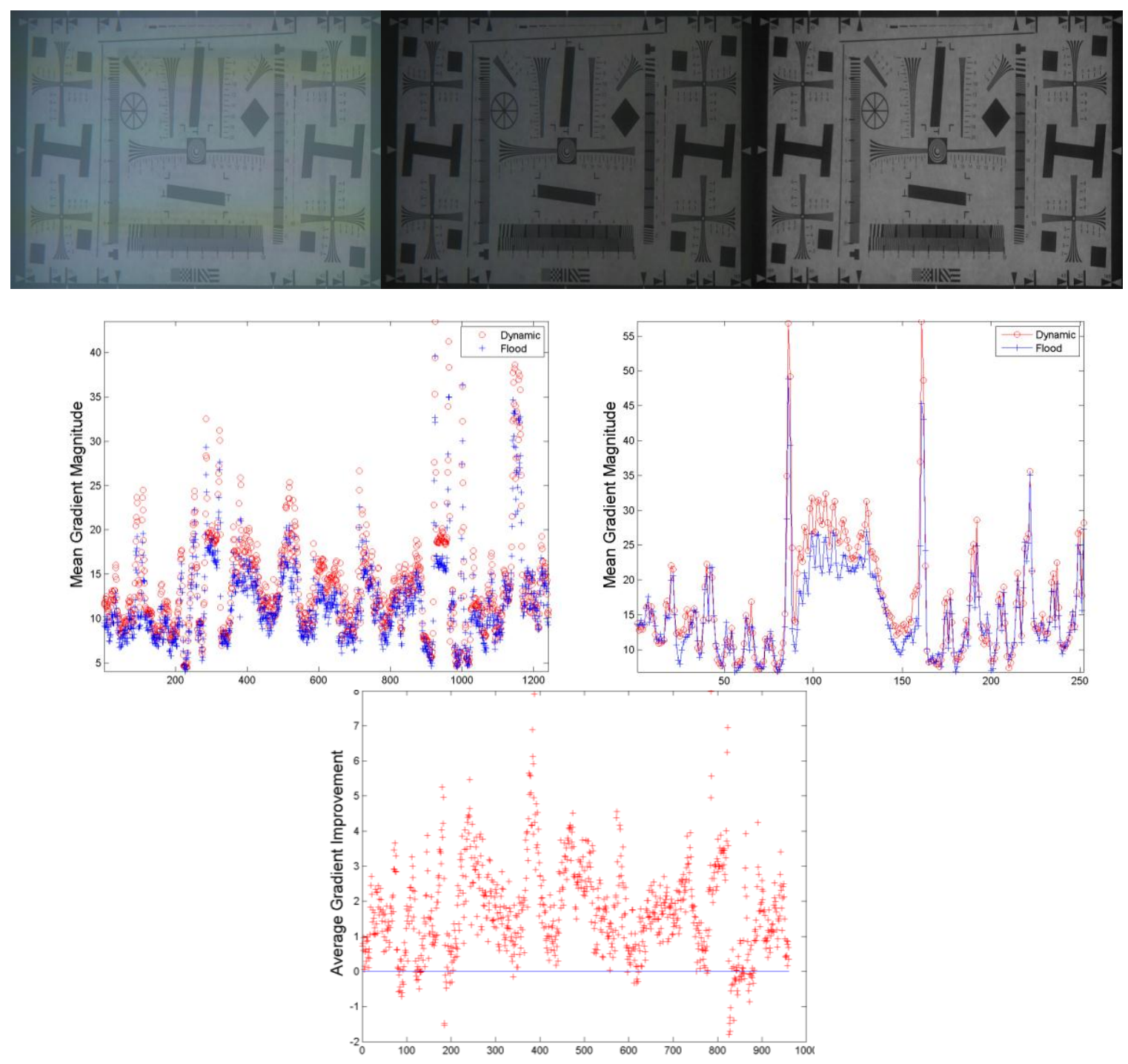

Minimum turbidity (<2.3 NTU) - These results are included for ease of comparison and analysis to the turbidity limit results provide below. 

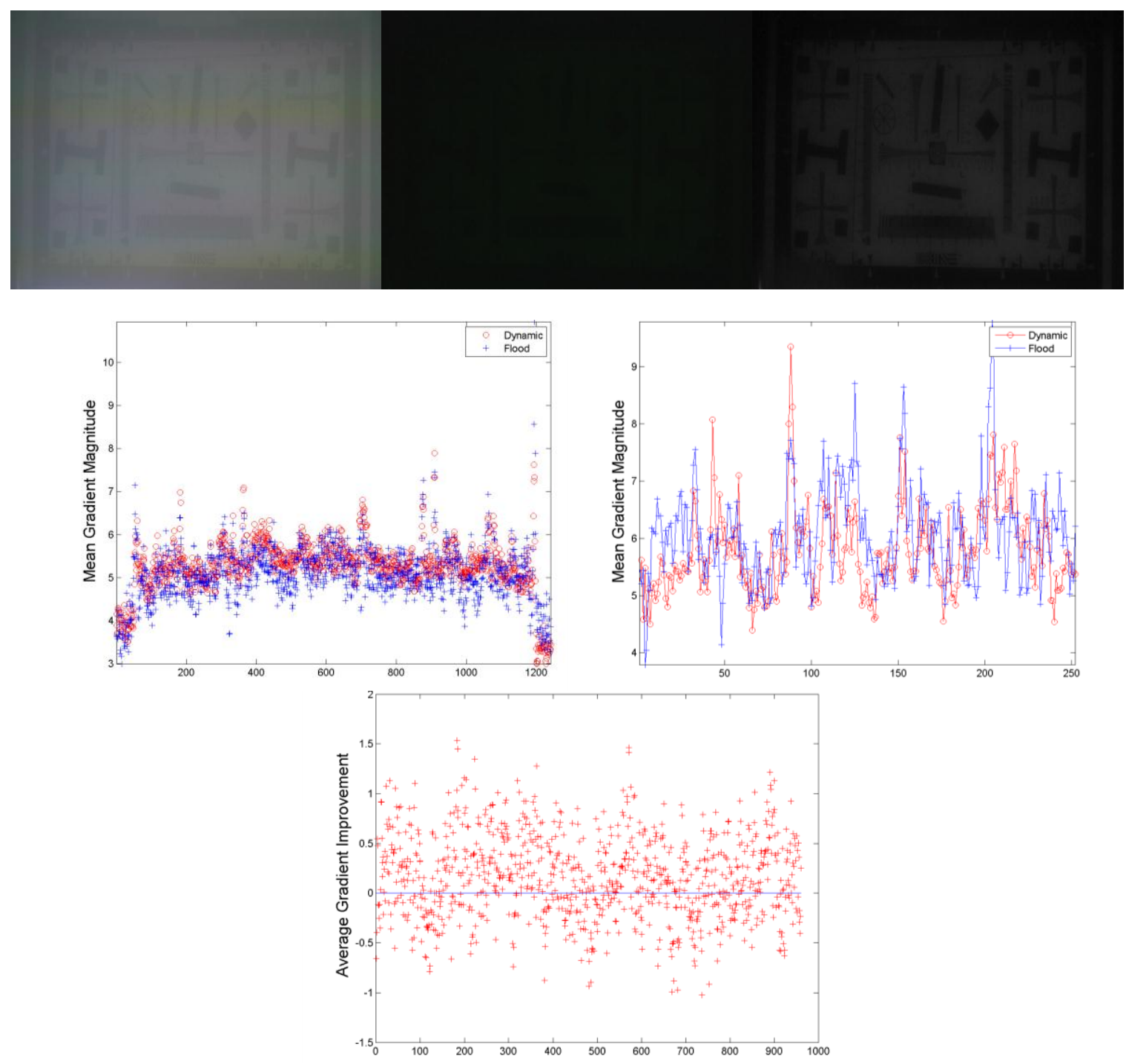

Turbidity of $\mathbf{5}$ NTU - The dynamic lighting system produced quality results with improved contrast in this turbidity. The specific results are provided in the previous section. 

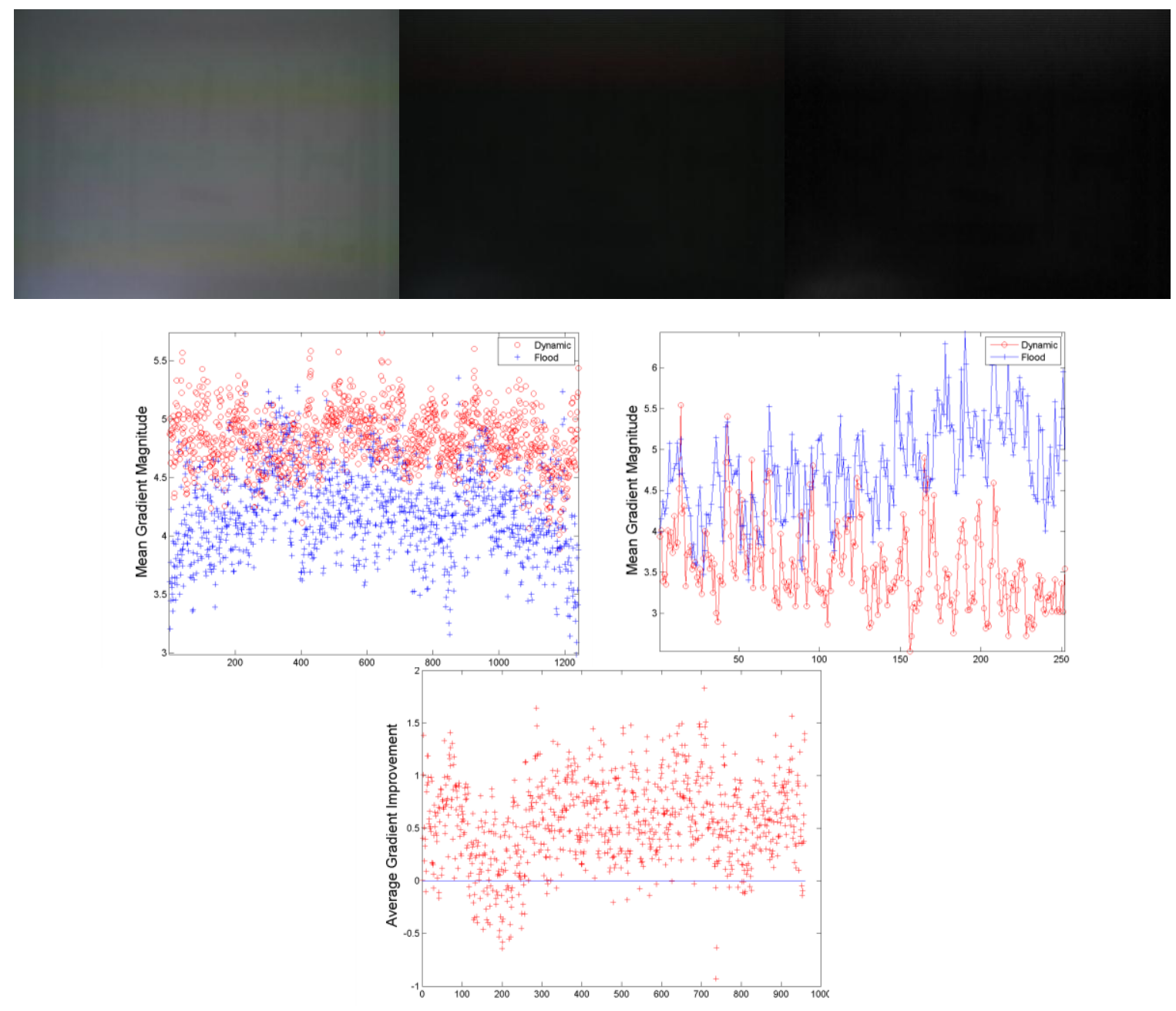

Turbidity of 7 NTU - A turbidity of 7 NTU represented the subjective limit of visualization of the resolution chart, by human eye, at $76.2 \mathrm{~cm}$. The system produced a marked increase in contrast, with a mean gradient change of 0.56 . The system worked and minimized the scatter apparent in the environment, but reproduces a minimal amount of the original scene information. 

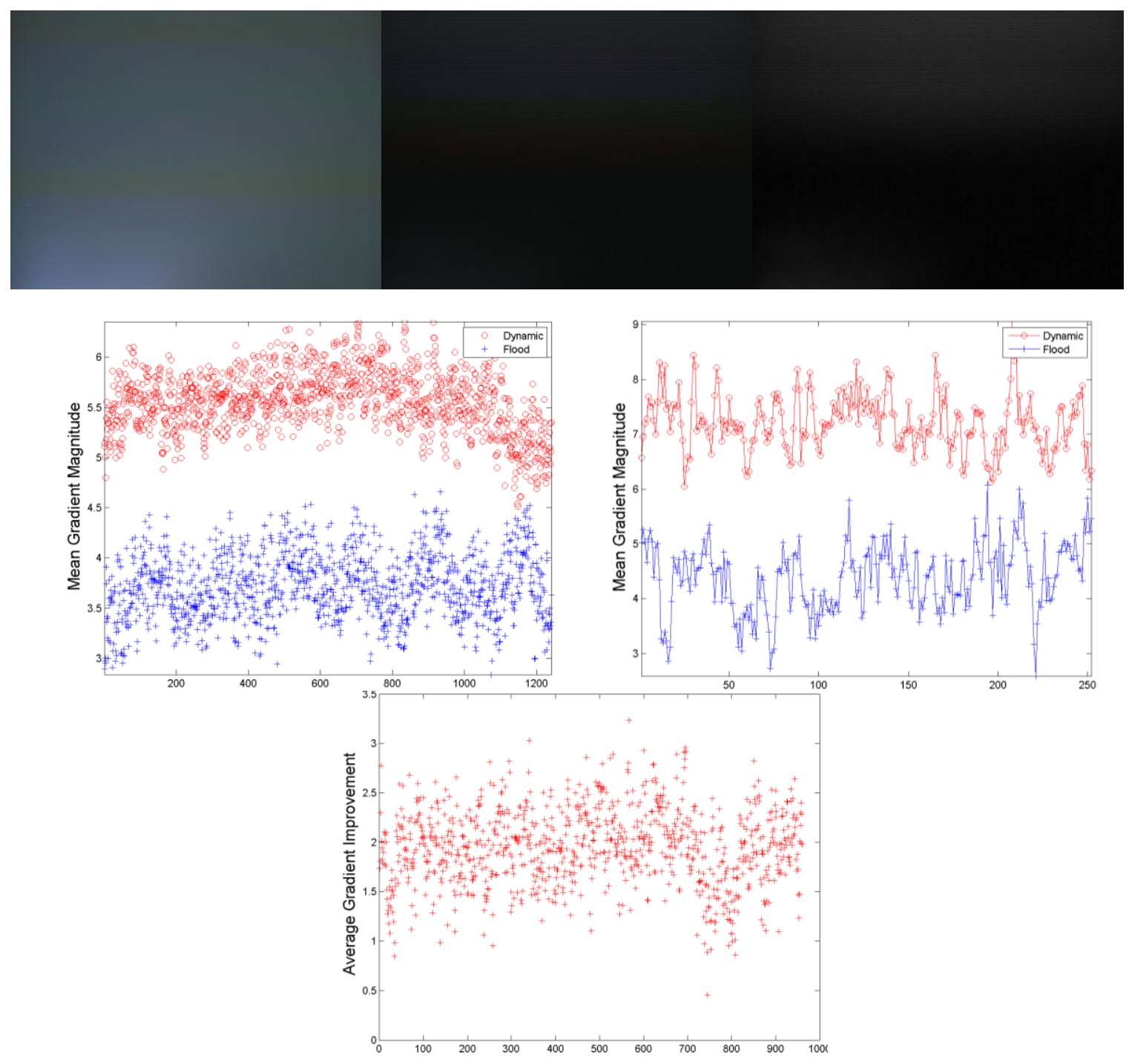

Turbidity of 9 NTU - At 9 NTU, the resolution chart was not visible to the naked eye at $76.2 \mathrm{~cm}$. This result shows a great increase in gradient magnitude throughout the entire image, but minimal reproduction of any scene information. The mean gradient change was just under 2 . This result mirrors those found in a 7 NTU environment. The environment coupled with system limitations overcame the ability to produce a satisfactory final image. 


\subsection{Interpretation of Results}

Overall the bench top design testing results showed promise and provided proof of the system effectiveness. In the first step of testing, I attempted to ascertain if the size of the dynamic lighting pattern made a difference in the overall quality of the final image. Upon analysis of the results, the 150 by 150 pixel white square, followed by the horizontal rectangle provided the most objective and subjective improvement in the reconstructed image. The horizontal rectangle merits a second look as it provided results on par with other much slower swept patterns even in greater turbidity (7 NTU vice 5). Although subjective and overall image gradient comparison showed some level of improvement for all of the patterns, the gradient comparison of the image center showed a more in depth story. For the 150 pixel sized square pattern, the overall contrast was improved and the scene information was adequately represented as well, especially when compared to the reference. A summary of testing results is provided in table 6 and figures 27 and 28.

\begin{tabular}{c|c|c|c|c}
\hline Pattern & $\begin{array}{c}\text { Sweep Speed } \\
(\mathrm{sec})\end{array}$ & $\begin{array}{c}\text { Illumination Area } \\
\left(\mathrm{pixel}^{2}\right)\end{array}$ & Gradient Change & Orig Information \\
\hline Square 75 & 63.8 & 5625 & -0.22 & minimal \\
\hline Square 100 & 41.3 & 10000 & 0.16 & yes \\
\hline Square 150 & 25.3 & 22500 & 0.97 & maximum \\
\hline Square 250 & 12.1 & 62500 & 0.09 & yes \\
\hline Vert Rectangle & 9.1 & 144000 & 0.24 & yes \\
\hline
\end{tabular}

Table 6 - Summary of bench top testing results. 


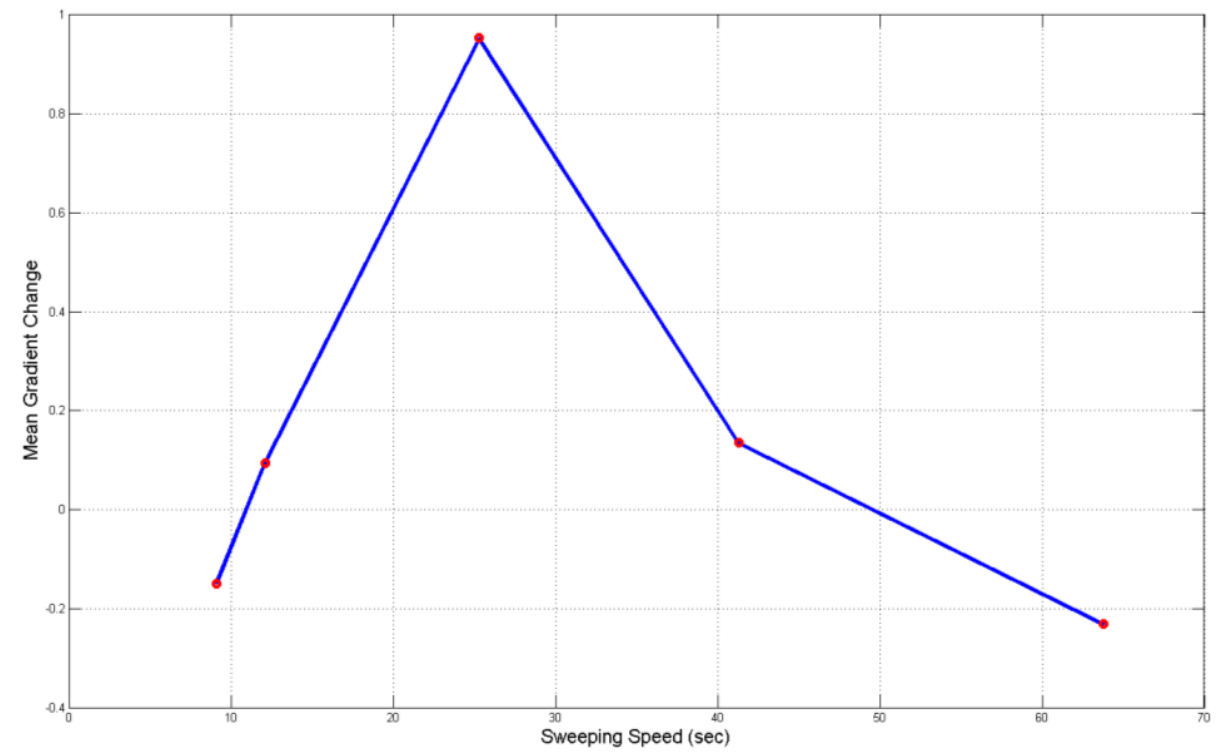

Figure 26 - Dynamic lighting sweeping speed effect on contrast. This graph represents the results found during bench top testing. The system shows a highly efficient area at 25 seconds of sweep speed. These results indicate the need for more fidelity in future testing concentrating on sweeping speed and resulting gradient improvement.

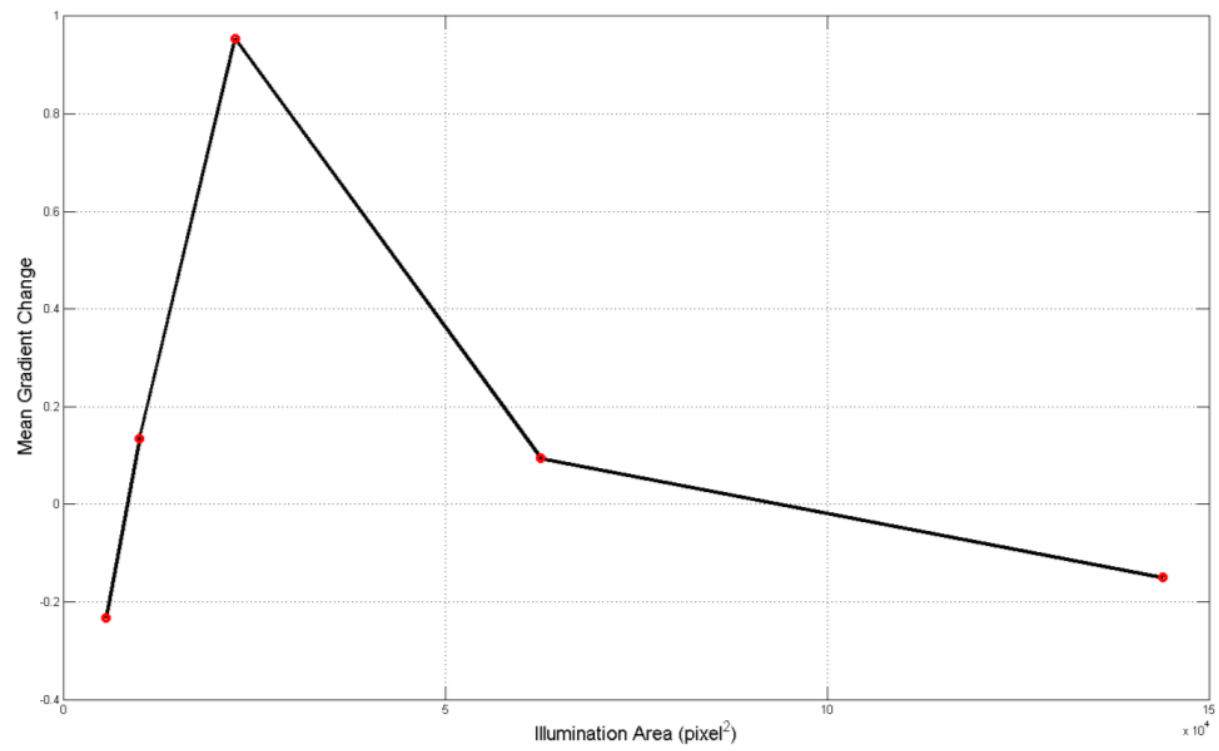

Figure 27 - Dynamic lighting area effect on contrast. This graph represents the results found during bench top testing. The system shows a gradient peak at approximately 10000 pixel $^{2}$ illumination area. These results indicate the need for more fidelity in future testing that concentrates on illumination area and resulting gradient improvement. 
The graphically represented results (figures 27 and 28) are provided to show the relationship between the resultant mean gradient increase and dynamic illumination sweeping speed and area. The sweeping speed during testing was determined by the size of the illumination pattern, therefore the two variables as presented are not independent and therefore show identical shapes when compared to mean gradient change. They are useful in that they provide a future way ahead for testing in that one must provide more in depth testing to more finely define the peak efficiency of the system and determine if the sweep speed and illumination area are actually dependent variables or not.

The turbidity limit testing was not so simple to interpret. At each of the turbidity environments, the system seemed to improve the overall quality of the scene image, especially at 9 NTU. These were misleading results, however, as although the overall gradient magnitude of the final images showed improvement, there was no image information provided in the final image. The turbidity limit testing proved that the system worked. The smaller scatter interaction volumes increased the final image contrast, but the low illumination intensity and increased attenuation in these highly turbid waters overcame the system advantages. This is not a show stopper, as these environments would overcome the limits of most optical illumination systems and provided a great frame of reference for future testing. 


\section{CHAPTER 5: CONCLUSIONS AND FUTURE WORK}

\subsection{Discussion}

The dynamic lighting system provided in this thesis was a successful first step in providing a new form of field deployable, low-cost, low-power supplemental imaging systems for the next generation of underwater vehicles. The testing and results proved that the system can successfully minimize the effects of scattering and was a viable source of turbid water images. With continued system design, refinement and testing, I believe that this avenue of imaging systems will be the future of the exploration into the depths of the sea, upper atmosphere and even space.

\subsection{Potential System Utilization}

One of the major advantages of this dynamic lighting system is that it has the inherent ability, as a virtually plug-and-play device, to be utilized as an additional or replacement processing and imaging system for numerous other oceanographic systems and / or vehicles. Some examples of the most seamless integration are with compressive structured light methods [Gu, et al], structured / coded lighting methods [Je, et al], [Scharstein \& Szeliski], [Salvi, et al] and [Narasimhan and Nayar], multi-plexed illumination [Park, et al], [Schechner, et al]

and of course a new and improved confocal imaging system [Levoy \& Singh] of 
which this project was based. This is not an exhaustive discussion, but does illustrate the usefulness of a low-power, black box type lighting system.

\subsection{Future Work}

Upon completion of the dynamic lighting system (DIS) and witnessing the great results, I quickly realized that this system had huge potential, especially with some added features and further universal simplifications. Some of the most important improvements and potential changes are listed below.

\subsubsection{Black Box Construct}

In order to be utilized by various existing oceanographic systems, the DIS was manufactured and designed as a plug and play system. In that sense, the system should be attached and aimed, with minimal electronic or physical attachments, and be ready to provide illumination. In theory, the DIS should contain just a pigtail to provide power and control signals in and get final postprocessed images out. These images would be available real time for onboard capture or upon dive / capture completion.

\subsubsection{Moving Platform Adaptation}

Although all of the DIS experimentation was conducted in a static environment, the ultimate intent was for implementation onto a moving underwater platform. As discussed by Levoy and Singh, a push broom panorama can easily be accomplished by simple control and software updates. The system would 
operate by sweeping the illumination pattern in the horizontal plane only, timed with the speed of the vehicle along the ocean bottom.

\subsubsection{Real-time Adaptability}

As discussed by Jaffe a system with real-time in-situ adaptability would be a major advance in oceanographic equipment [Jaffe 2010]. This in-situ control was an original design goal of the dynamic lighting system (DLS) that did not come to fruition. The next generation dynamic lighting system would include a real time imaging system onboard that would measure a specific environmental QA metric that would be used to switch between onboard flood-lighting and sweeping illumination.

The new system (DLS 2.0) was built, but required software updates that would allow control of illumination based on the presence of scatter. The drawback to in-situ control is the more in depth integration between the lighting control system and the existing platform, leading to a decreased level of black box design. The DIS 2.0, with potential onboard scatter detection camera, is shown in figure 29.

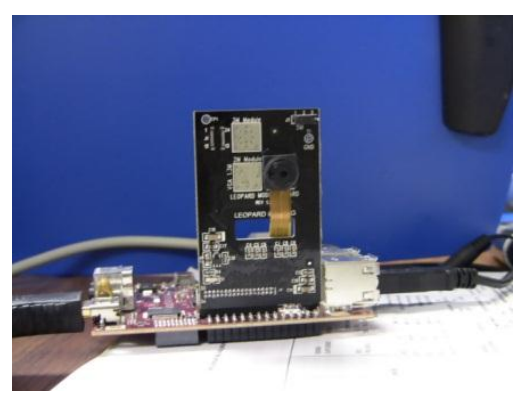

Figure 28 - Dynamic lighting system 2.0 (DLS 2.0) 


\subsubsection{Additional Design Testing}

The most important aspect for future work is the addition of more specific and robust design testing. Future testing should include additional pattern shapes, pattern sweep speeds and more fidelity in pattern size and turbidity limits. This increased level of experimentation will allow the most efficient design possible and may provide clues into the efficiency of different pattern / speed / shape combinations based on turbidity conditions.

\subsubsection{Deployment Testing}

The most important work that still needs completion is the testing of the system in an actual deployed environment. Although not completed at the completion of this thesis, I have no doubt that the system, with minor changes, will succeed as a field deployed system. 


\section{CHAPTER 6: REFERENCE}

\subsection{Appendices}

6.1.1 Appendix A - List of Equations

(1) $I(r)=I\left(r_{o}\right) e^{-e\left(r-r_{o}\right)}=I\left(r_{o}\right) e^{-(a+b)\left(r-r_{o}\right)}$

- Simple exponential law to explain light attenuation via absorption $(a)$ and scattering $(b)$

(2) $b=\int_{4 \pi} b(\theta) d \omega$

- Scattering coefficient (scatter per unit meter, per unit steradian) described by angular dependent scattering $(b(\theta))$ coefficient and solid angle $(\omega)$

- Integral is taken over all $4 \pi$ steradians

(3) $\frac{1}{4 \pi} \int_{4 \pi} \beta(\theta) d \omega=1$

- Volume scatter or phase function; probability that a photon can be scattered into solid angle $d \omega$

- $\beta(\theta)$ is normalized and scaled version of $b(\theta)$ 


\subsubsection{Appendix B - Definitions}

All definitions are from [Morel \& Smith] unless noted

Average cosines $\left(\bar{\mu}, \overline{\mu_{d}}, \overline{\mu_{u}}\right)$

- The ratio of the net (downward) irradiance to scalar irradiance

\section{Absorptance $(A)$}

- The ratio of the radiant flux $\left(\Phi_{a}\right)$ lost from a beam by means of absorption, to the incident flux $\left(\Phi_{i}\right)^{6}$

Absorption coefficient $(a)$

- The absorptance of an infinitesimally thin layer of the medium normal to the beam, divided by the thickness of the layer

- Sl unit is $m^{-1}$

\section{Attenuance $(C)$}

- The ratio of the radiant flux lost from a beam of infinitesimal width by means of absorption and scattering to the incident flux

Attenuation Coefficient (c)

- Measure of the light loss from the combined effects of scattering and absorption over a unit length of travel in an attenuation medium

- $c=a+b$

Downward irradiance $\left(E_{d}\right)$

- The radiant flux on an infinitesimal element of the upper face (i.e., facing zenith) of a horizontal surface containing the point being considered, divided by the area of the element

- Si unit is $W \cdot m^{-2}$

Irradiance (at a point of a surface) (E)

- The radiant flux incident on an infinitesimal element of a surface containing the point under consideration, divided by the area of that element

- SI unit is $W \cdot m^{-2}$

\footnotetext{
${ }^{6}$ Original variable is $\Phi_{o}$, was changed for consistency with remainder of text
} 
Lumen

- The SI unit of luminous flux

- The amount of light emitted per second in a unit solid angle of one steradian from a uniform source of one candela

- from http://dictionary.reference.com/browse/lumen

Mie Scattering

- Scattering of light by particles the same size or larger than the wavelength of the incident light

Nephelometric turbidity unit (NTU)

- A measure of turbidity in a water sample, roughly equivalent to Formazin turbidity unit (FTU) and Jackson turbidity unit (JTU)

- Water containing 1 milligram of finely divided silica per liter has a turbidity of 1 NTU

- from http://www.sizes.com/units/nephelometric unit.htm

Photon

- A quantum of electromagnetic radiation that has an energy equal to the product of the frequency of the radiation by the Plank's constant $h$

- $\quad h=(6.626176 \pm 0.000036) x 10^{-34} \mathrm{~J}[\mathrm{~S}$

Probability of photon survival $\left(\omega_{o}, \omega\right)$

- The ratio of the scattering coefficient to the attenuation coefficient

- Sometimes known as single-scattering albedo

Radiance (L)

- Radiant flux in a given direction per unit solid angle per unit projected area

- SI unit is $W \cdot m^{-2} \cdot s r^{-1}$

Radiant Energy $(W, Q)$

- Energy emitted, transferred, or received as radiation

- SI unit is Joules $(\mathrm{J})$

Radian Flux $(\Phi, F)$

- The time rate of flow of radiant energy

- SI unit is Watts (W) 
Radiant Intensity ( $I$ )

- The radiant flux emitted by a point source, or by an element of an extended source, in an infinitesimal cone containing the given direction, divided by that element of solid angle

- SI unit is Watts per steradian $\left(W \cdot s r^{-1}\right)$

Rayleigh scattering

- Elastic scattering of light by particles much smaller than the wavelength of the incident light

Refractive Index $(\eta)$

- The ratio of the velocity of electromagnetic radiation, in vacuum, to the phase velocity of electromagnetic radiation of a specified frequency medium

- dimensionless

Scalar irradiance $\left(E_{o}\right)$

- The integral of radiance distribution at a point over all directions about the point

- SI unit is $W \cdot m^{-2}$

Scatterance $(B)$

- The ratio of the radiant flux $\left(\Phi_{b}\right)$ scattered from a beam, to the incident flux $\left(\Phi_{i}\right)$

Scattering Coefficient (b)

- Superposition of all scattering events at all angles through the volume scattering function, $\beta(\theta)$

- $\quad b=2 \pi \int_{0}^{\pi} \beta(\theta) \sin \theta d \theta$

Solid Angle

- The angle that, seen from the center of a sphere, includes a given area on the surface of that sphere. The value of a solid angle is numerically equal to the size of that area divided by the square of the radius of the sphere 
Steradian (sr)

- The SI unit for solid angle

- Used to describe two-dimensional angular spans in three-dimensional space, analogous to the way in which the radian describes angles in a plane

- from http://en.wikipedia.org/wiki/Steradian

Total attenuation coefficient $(c)$

- The attenuance of an infinitesimally thin layer of the medium normal to the beam, divided by the thickness of the layer

- SI unit is $m^{-1}$

Total scattering coefficient $(b)$

- The scatterance of an infinitesimally thin layer of the medium normal to the beam, divided by the thickness of the layer

- Alternative: the integral over all directions of the volume scattering function

- Includes the backward and forward scattering coefficients

- SI unit is $m^{-1}$

Upward irradiance $\left(E_{u}\right)$

- The radiant flux incident on an infinitesimal element of the lower face (i.e., facing nadir) of a horizontal surface containing the point being considered, divided by the area of the element

- Si unit is $W \cdot m^{-2}$

Volume scattering function $(\beta(\theta))$

- The radiant intensity, from a volume element in a given direction, per unit of irradiance on the cross-section of the volume and per unit volume

- Gives the probability for a ray of light to be deviated of an angle $\theta$ from its direction of propagation

- SI unit is $m^{-1} s r^{-1}$

Wavelength $(\lambda)$

- Distance between two successive points of a periodic wave in the direction of propagation, for which oscillation has the same phase

- SI unit is meter $(\mathrm{m})$ 
6.1.3 Appendix C - System Component Specifications

Texas Instruments DLP® Pico ${ }^{\mathrm{TM}}$ Projector Development Kit

Designed to interface with the Beagle Board to expedite development.

(from http://focus.ti.com/general/docs/gencontent.tsp?contentld=52770)

Resolution: $\quad$ 0.17-inch HVGA

Brightness: $\quad 7$ ANSI lumens

Contrast ratio: $\quad 1000: 1$

Throw ratio: $\quad 1.89$

Light source: $\quad$ Solid-state 3 LED

Dimension: $\quad 44.8 \times 67.4 \times 14.2 \mathrm{~mm}$

Optoma Pico Projectors (from http://optimausa.com/products/detail)

PK201

Display: $\quad$ DLP

Resolution: $\quad$ Native WVGA (854 x 480), maximum 1080i

Brightness: 20 ANSI lumens

Contrast ratio: $\quad 2000: 1$ (full on / full off)

Light source: $\quad$ LED (RGB)

Throw ratio: $\quad$ 2.2:1 (Distance / Width)

Dimensions: $\quad 60.6 \times 16.6 \times 117.7 \mathrm{~mm}$ 
Optima Pico Projectors continued

PK301

Display: $\quad$ DLP

Resolution: $\quad$ Native WVGA (854 x 480), maximum 1080i

Brightness: $\quad 50$ ANSI lumens

Contrast ratio: $\quad 2000: 1$ (full on / full off)

Light source: $\quad$ LED (RGB)

Throw ratio: $\quad$ 1.8:1 (Distance / Width)

Dimensions: $\quad 120.1 \times 29.7 \times 69.8 \mathrm{~mm}$

Beagle Board (from http://beagleboard.org)

The Beagle Board in a low-cost, fan-less single board computer that provides laptop performance and expandability without the size, expense or noise of a typical desktop machine.

Revision C3

Processor: OMAP3530DCBB72 at $750 \mathrm{MHz}$

Memory: $\quad$ Micron 2Gb NAND (256MB)

Micron 2Gb MDDR SDRAM (256MB)

Storage: $\quad 6$ in $1 \mathrm{SD} / \mathrm{MMC} / \mathrm{SDIO}, 4 / 8$ bit support, dual voltage

Video: $\quad$ DVI-D and S-Video

Audio: $\quad$ Stereo in and out ports $(3.5 \mathrm{~mm} \mathrm{~L}+\mathrm{R})$

Power: $\quad$ USB and DC (5V @ 350ma typical)

Expansion: High Speed USB (x1), UART, McBSP, McSPI, I2C, GPIO, MMC, LCD connectors ( $\mathrm{x} 2)$ and PWM 


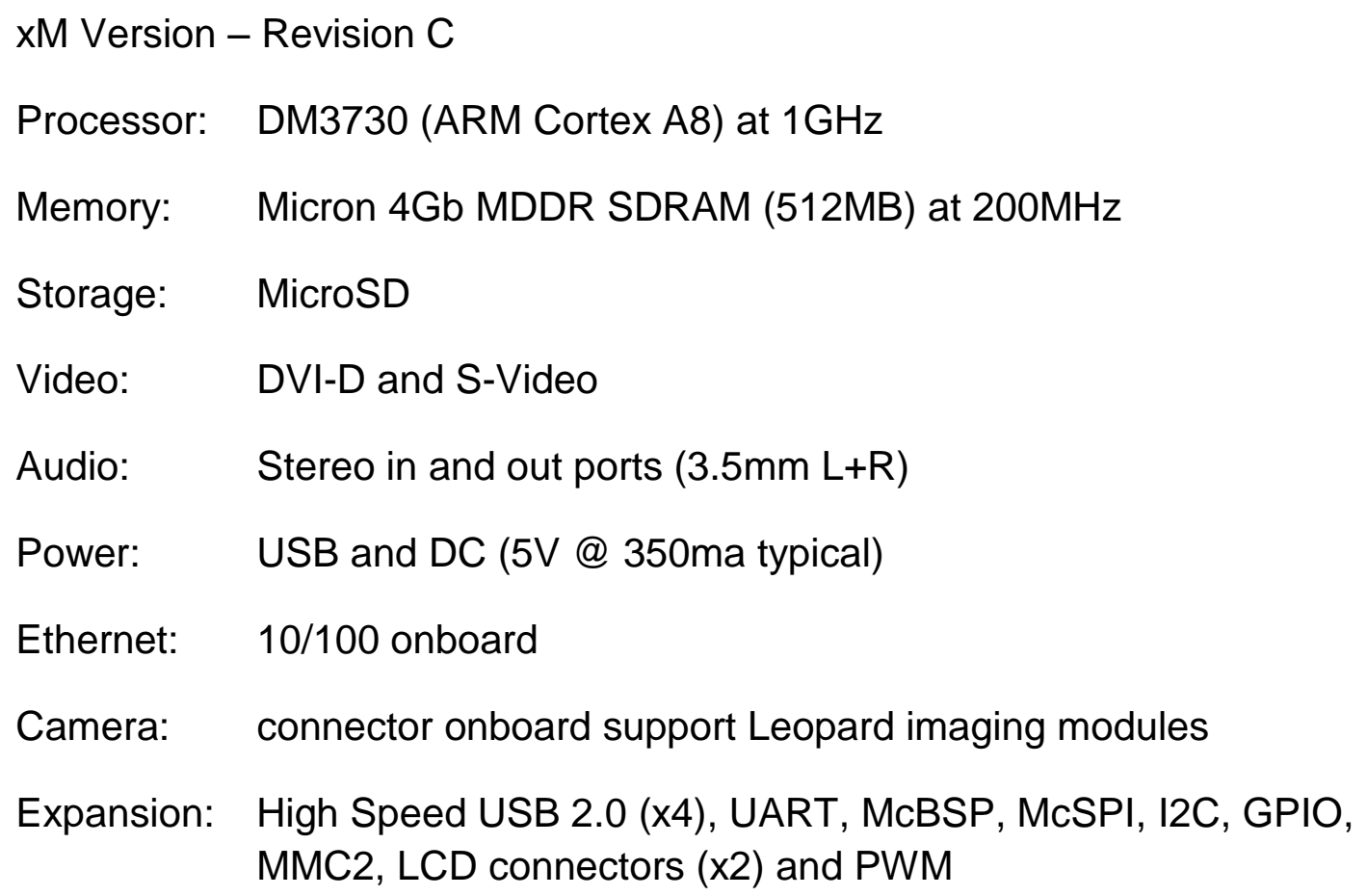
MMC2, LCD connectors (x2) and PWM

Zippy2 expansion board (from http://beagleboard.org)

10/100BaseT Ethernet

SD/MMC interface

RS232 Serial interface

Real-Time clock with battery backup

I2C interface

AT24C01 Serial EEPROM for board identification 


\subsection{Works Cited}

[1] Bazeille, S., Quidu, I., Jaulin, L. and Malkasse, J., (2006). "Automatic Underwater Image Pre-Processing," CMM 2006, retrieved from http://hal.archives-ouvertes.fr/hal-00504893/en/

[2] Bradley, A. M., Feezor, M. D., Singh, H., and Sorrell, F. Y., (2001). "Power Systems for Autonomous Underwater Vehicles," IEEE Journal of Oceanic Engineering, 26(4), 526-538.

[3] Chang, C. Y., Flitton, J. C., Hopcraft, K. I., Jakeman, E., Jordan, D. L. and Walker, J. G., (2003). "Improving visibility depth in passive underwater imaging by use of polarization," Applied Optics, 42(15), 2794-2803

[4] Duntley, S. Q., (1962). "Light in the Sea," Visibility Laboratory, Scripps Institution of Oceanography, La Jolla, California

[5] Garcia, R., Nicosevici, T. and Cufi X., (2002). "On the Way to Solve Lighting Problems in Underwater Imaging," Proceedings of the IEEE Ocean Conference Record, 2, 1177-1180

[6] Gilbert, G. D. and Pernicka, J. C.,(1967). "Improvement of Underwater Visibility by Reduction of Backscatter with a Circular Polarization Technique," Applied Optics, 6(4), 741-746

[7] Gu, J., Nayar, S. K., Ginspun, E., Belhumeur, P.N., and Ramamoorthi, R., (2008). "Compressive Structured Light for Recovering Inhomogeneous Participating Media," European Conference on Computer Vision (ECCV) 2008. Retrieved from https://www.cs.columbia.edu/CAVE/publications

[8] Guitierrez, D., Seron, F., Munoz, A. and Anson, O., (2008). "Visualizing Underwater Ocean Optics," EUROGRAPHICS 2008, 27(2), 10 pages.

[9] Gupta, M., Narasimhan, S. G. and Schechner, Y. Y., (2008). "On Cotrolling Light Transport in Poor Visibility Environments," In ACM SIGGRAPAPH ASIA 2008 courses, Article 68, doi:10.1145/1508044.1508112

[10] Han, H., Kim, D., Park, R. and Sim, D., (2008). "Visual Quality Metric Using One-Dimensional Histograms of Motion Vectors," Proc. of SPIE 6808, 68088H (2008); doi:10.1117/12.766948

[11] Holst, G. C., (2011). "Imaging system fundamentals," Optical Engineering, 50(5), $1-10$. 
[12] Hou, W., Woods, S., Goode, W., Jorosz, E. and Weidemann, A. D., (2011). "Impacts of optical turbulence on underwater imaging," Proc. SPIE 2011, 8030, 1-9, doi: 10.1117/12.883114

[13] Hou, W. and Weidemann, A. D., (2007). "Objectively assessing underwater image quality for the purpose of automated restoration," Proc. of SPIE, vol. 6575, doi:10.1117/12.717789

[14] Hou, W., Gray, D. J., Weidemann, A. D., Fournier, G. R., and Forand, J. L., (2007). "Automated underwater image restoration and retrieval of related optical properties," IGARSS 2007, 1889-1892, doi: 10.1109/IGARSS.2007.4423193

[15] Jaffe, J. S., (2010). "Enhanced Extended Range Underwater Imaging via Structured Illumination," Optics Express, 18, 12328-12340

[16] Jaffe, J. S., (1995). "Monte Carlo modeling of underwater-image formation: validity of the linear and small-angle approximations," Applied Optics, 34(24), 5413-5421

[17] Jaffe, J. S., Moore, K. D., McLean J., and Strand, M.P., (2001). "Underwater Optical Imaging: Status and Prospects," Oceanography, 14(3), 66-75.

[18] Je, C., Lee, S. W. and Park, R., (2004). "High-Contrast Color-Stripe Pattern for Rapid Structured-Light Range Imaging," ECCV 2004, 95-107

[19] Jeong, T. Kim, Y. and Lee C., (2010). "No-reference image-quality metric based on blur radius and visual blockiness," Optical Engineering, 49(4), 1 -9

[20] Kirk, J. T. O., (1991). "Volume scatter function, average cosines, and the underwater light field," Limnol Oceanogr, 36(3), 455-467

[21] Kocak, D. M., Dalgleish, F. R., Caimi, F. M. and Schechner, Y. Y., (2008). "A Focus on Recent Developments and Trends in Underwater Imaging," Marine Technology Society Journal, 42(1), 52-67

[22] Kopelevisch, O. "Topic 7: Oceanic Optical Properties and Models," retrieved from http://www.ioccg.org/training/turkey/DrKopelovich_Topic7.pdf

[23] Kulp, T. J., Garvis, D., Kennedy, R., Salmon, T. and Cooper, K., (1995). "Development and testing of a synchronous-scanning underwater imaging system capable of rapid two-dimensional frame imaging," Applied Optics, 32, 3520-3530 
[24] Larson, E. C. and Chandler, D. M., (2010). "Most apparent distortion: fullreference image quality assessment and the role of strategy," Journal of Electronic Imaging, 19(1), 1-21

[25] Levoy, M. and Singh, H., (2009). "Improving underwater vision using confocal imaging," Stanford Computer Graphics Laboratory Technical Memo, 2009-001

[26] Liu, M. and Yang, X., (2009). "Image quality assessment using contourlet transform," Optical Engineering, 48(10), 107201, 1-10

[27] Maffione, R., A., (2001). "Evolution and Revolution in Measuring Ocean Optical Properties," Oceanography, 14(3), 9-14

[28] McFarlane, J. R., (2008). "Tethered and Untethered Vehicles: The Future is the Past" Oceans 2008, 1-4. doi:10.1109/OCEANS.2008.5151918

[29] Miras, D., (2002), "A Survey on Netword QoS Needs of Advanced Internet Applications," Working Group Paper, retrieved from https://qos.internet2.edu/wg/apps/fellowship/Docs/Internet2AppsQoSNeeds.ht $\mathrm{ml}$

[30] Mobley, C. D., (2010). "Optical Properties of Water," in Handbook of Optics, Volume 1, 2010 McGraw-Hill

[31] Mobley, C. D., (2004). "Light and Water: Radiative Transfer in Natural Waters," June 2004, retrieved from http://misclab.umeoce.maine.edu/education/Light\&Water/

[32] Morel, A. and Smith, R. C., (1982), "Terminology and Units in Optical Oceanography," Marine Geodesy, 5(4), 335-349

[33] Mullen, L., Cochenour, B., Laux, A. and Alley, D., (2011). "Optical modulation techniques for underwater detection, ranging and imaging," SPIE 2011, 8030, 1-9

[34] Myre, E. and Shaw, R., (2006), "The Turbidity Tube: Simple and Accurate Measurement of Turbidity in the Field," CE 5993 Course Paper, Department of Civil and Environmental Engineering, Michigan Technological University. 
[35] Narasimhan, S. G., Nayar, S. K., Sun, B. and Koppal, S. J. (2005). "Structured Light in Scattering Media," Computer Vision 2005. International Conference of Computer Vision (ICCV) 2005, 1, 420-427.

doi:10.1109/ICCV.2005.232

[36] Narasimhan, S. G. and Nayar, S. K., (2005). "Structured Light Methods for Underwater Imaging: Light Stripe Scanning and Photometric Stereo," IEEE / MTS Oceans 2005, 2610-2617

[37] Nayar, S. K., (2011). "Computational Cameras: Approches, Benefits and Limits," Technical Report, retrieved from http://handle.dtic.mil/100.2/ADA536114

[38] Nayar, S. K., Krishnan, G., Grossberg, M. D. and Raskar, R., (2006). "Fast Separation of Direct and Global Components of a Scene using High Frequency Illumination," ACM SIGGRAPH 2006, 25(3), 935-944, doi:10.1145/1179352.1141977

[39] Ouni, S., Chambah, M., Herbin, M and Zagrouba, E., (2008). "Are Existing Procedures Enough? Image and Video Quality Assessment: Review of Subjective and Objective Metrics," Proc. of SPIE 6808, 68080Q(2008)

[40] Owen, R. W., (1973). "The Effect of Particles on Light Scattering in the Sea," Journal of the Oceanographic Society of Japan, 29, 171-184

[41] Park, J., Lee, M., Grossberg, M. D. and Nayar, S. K., (2007). "Multispectral Imaging Using Multiplexed Illumination," ICCV 2007, 1-8

[42] Petzold, T. J., (1972). "Volume Scattering Functions for Selected Ocean Waters, Scripps Institution of Oceanography, La Jolla, California

[43] Salvi, J., Pages, J. and Batlle, J., (2003). "Pattern codification strategies in structure light systems," Pattern Recognition, 37 (2004), 827-849

[44] Scharstein, D., and Szeliski, R. "High-Accuracy Stereo Depth Maps Using Structured Light," CPVR 2003, 1, 195-202. doi:10.1109/CVPR.2003.1211354

[45] Schechner, Y. Y. and Karpel, N., (2004). "Clear Underwater Vision," CVPR 2004, 1, 536-543, doi: 10.1109/CVPR.2004.1315078

[46] Schechner, Y. Y., Nayar, S. K. and Belhumeur, P. N., (2003), "A Theory of Multiplexed Illumination," ICCV 2003, 2, 808, doi:10.1109/ICCV.2003.1238431 
[47] Schettini, R. and Corchs, S., (2010). "Underwater Image Processing: State of the Art of Restoration and Image Enhancement Methods," EURASIP Journal on Advances in Signal Processing, vol. 2010, Article ID 746052, 14 pages, 2010. doi:10.1155/2010/746052

[48] Singh, H., Howland, J., and Pizarro, O., (2004). "Advances in Large-Area Photomosaicking Underwater," IEEE Journal of Oceanic Engineering, 29(3), 872-886

[49] Smith, R. C. and Baker, K. S., (1981). "Optical properties of the clearest natural waters," Applied Optics, 20(2), 177-184

[50] Strand, M. P., (1995). "Underwater electro-optical system for mine identification," Proceedings of SPIE, 2496, 487-497

[51] Sydor, M., Gould, R. W., Arnone, R. A., Haltrin, V. I., and Goode W., (2004). "Uniqueness in remote sensing of the inherent optical properties of ocean water," Applied Optics, 43, 2156-2162

[52] Tyo, J. S., Rowe, M. P., Pugh, E. N. and Engheta, N., (1996). "Target detection in optically scattering media by polariztion-difference imaging," Applied Optics, 35(11), 1855-1870

[53] Walker, J. G., Chang, P. C. Y. and Hopcraft, K. I., (2000). "Visibility depth improvement in active polarization imaging in scattering media," Applied Optics, 39(27), 4933-4941

[54] Wang, R., Cui, Y. and Yuan, Y., (2011). "Image quality assessment using full-parameter singular value decomposition," Optical Engineering, 50(5), 1-9

[55] Woods Hole Oceanographic Institution (WHOI), (2011)., "WHOI-led Team Locates Air France Wreckage," WHOI News Release, 2011, retrieved from http://www. whoi.edu/page.. o ?pid=7545\&tid=282\&cid=96189\&ct $=162$

[56] Zhang, L., Hu, L, and He M., (2009). "Scattering by pure seawater: Effect of salinity," Optics Express, 17(7), 5689-5710 